$$
N G R-09.015-082
$$

\title{
THIRD-ORDER \\ SOLUTION OF AN \\ ARTIFICIAL-SATELLITE \\ THEORY
}

\section{HIROSHI KINOSHITA}

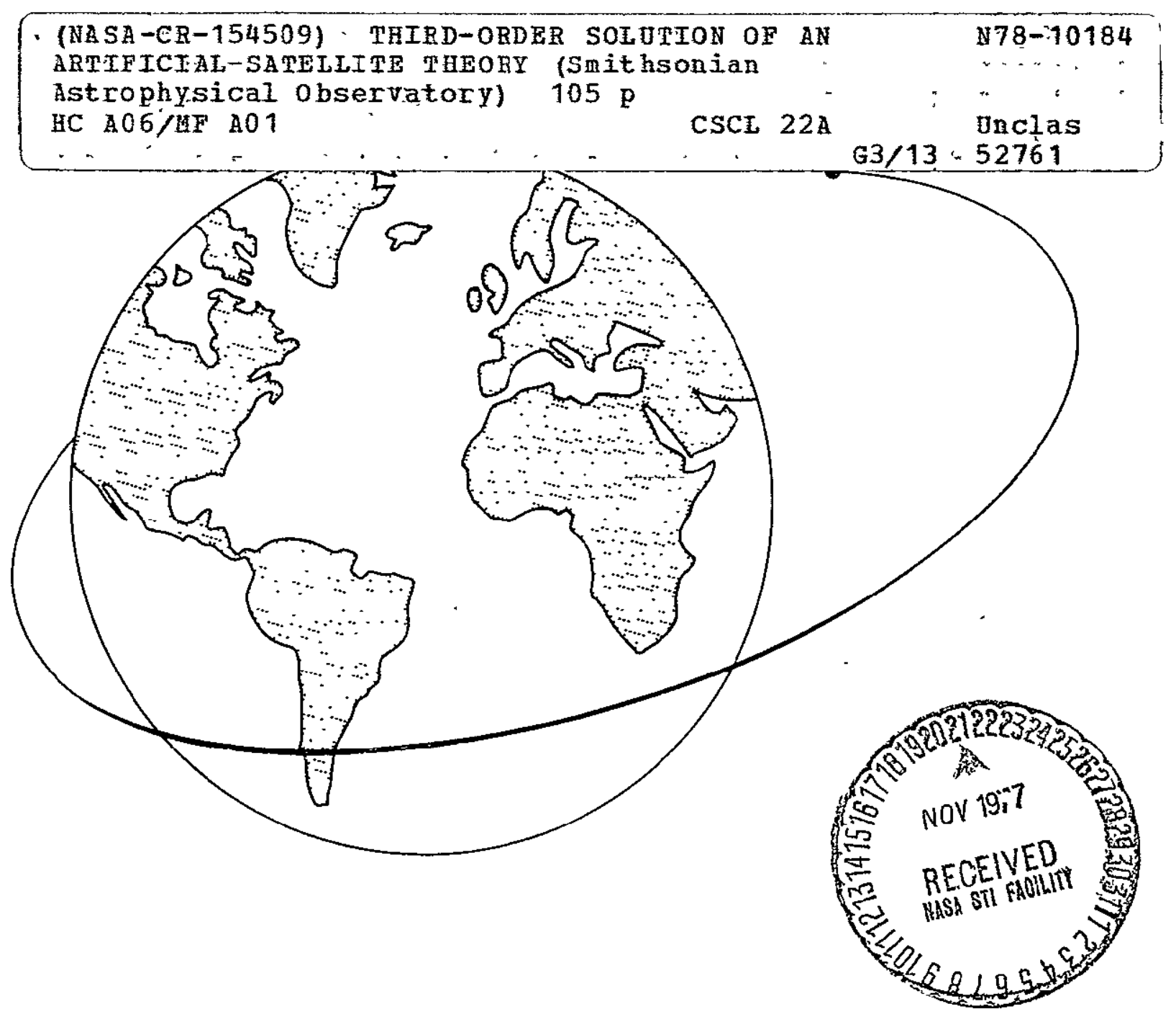

Smithsonian Astrophysical Observatory SPECIAL REPORT 379 
Research in Space Science

SAO Special Report No. 379

THIRD-ORDER SOLUTION OF AN ARTIFICIAL-SATELLITE THEORY

Hiroshi Kinoshita

JuTy 27, 1977

Smithsonian Institution

Astrophysical Observatory

Cambridge, Massachusetts 02138 
TABLE OF CONTENTS

Section Page

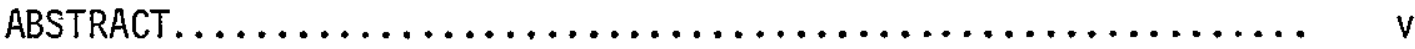

1 INTRODUCTION........................................

2 OUTLINE OF THE METHOD OF SOLUTION........................ 3

3 COMPUTATION ${ }^{\circ}$ OF POSITION AND VELOCITY..................... 15

4 ANALYTICAL AND NUMERICAL CHECK....................... 19

ACKNOWLEDGMENTS ................................. 29

REFERENCES....................................... 31

Appendix

A EXPRESSIONS OF $S, F^{*}, S^{*}$, AND $F^{* *} \ldots \ldots \ldots \ldots \ldots \ldots \ldots \ldots \ldots \ldots \ldots \ldots \ldots \ldots$

A. 1 Expressions of S.............................. A-1

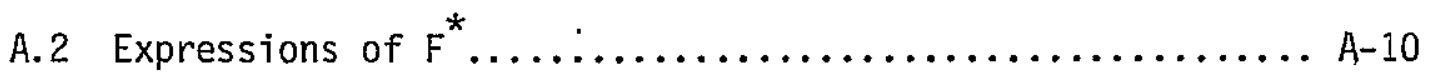

A. 3 Expressions of $S^{*} \ldots \ldots \ldots \ldots \ldots \ldots \ldots \ldots \ldots \ldots \ldots \ldots, A, 12$

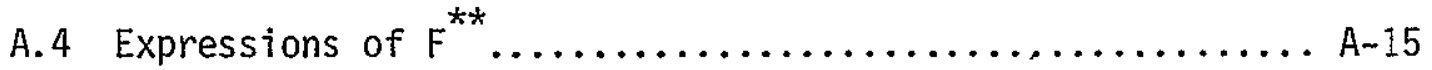

B EXPRESSTONS OF SHORT-PERIOD, LONG-PERIOD, AND ȘECULAR

PERTURBATIONS . . . . . . . .

B.1 Second-Order Short-Period Perturbations due to $\mathrm{J}_{2}^{2} \ldots \ldots$ B-1

B.2 Third-Order Short-Period Perturbations due to $J_{2}^{3} \ldots \ldots \ldots, B-13$

B.3 Third-Order Short-Period Perturbations due to $\mathrm{J}_{2} \mathrm{~J}_{3} \ldots \ldots$. B-24

B.4 Third-Order Short-Period Perturbations due to $\mathrm{J}_{2} \mathrm{~J}_{4} \ldots \ldots$... B-33

B.5 Second-Order Long-Period Perturbations............... B-44

B.6 Third-Order Long-Period Perturbations................ B-48

B.7 Secular Perturbations........................... B-52 


\section{ILLUSTRATIONS}

$\underline{\text { Page }}$

1 In-track, along-track, and across-track prediction errors of the third-order solution for Starlette plotted over two revolutions......................................... 23

2 The same as Figure 1 plotted over about 600 revolutions........ 24

3 In-track, along-track, and across-track prediction errors of the third-order solution for Anna 1B plotted over 860 revolutions........................................ 26

4 The same as Figure 3 plotted over about 2700 revolutions....... 27

TABLES

Page

1 Numbers of terms in the determining function $s_{3} \ldots \ldots \ldots \ldots \ldots, 10$

2 Numbers of terms in the determining function $s_{3}^{*} \ldots \ldots \ldots \ldots, 11$ 
A third-order solution is developed for the motions of artificial satellites moving in the gravitational field of the earth, whose poten-. tial includes the second-, third-, and fourth-order zonal harmonics. Third-order periodic perturbations with fourth-order secular perturbations are derived by Hori's perturbation method. All quantities are expanded into power series of the eccentricity, but the. solution is obtained so as to be closed with respect to the inclination. A comparison with the results of numerical integration of the equations of motion indicates that the solution can predict the position of a close-earth, sma11-eccentricity satellite with an accuracy of better than $1 \mathrm{~cm}$ over a period of 1 month. 
Hiroshi Kinoshita

\section{INTRODUCTION}

Second-order theories of artificial satellites have been established by many authors during the past 15 years; an excellent review is given by Hori and Kozai (1975). A third-order solution was derived by Deprit and Rom (1970), but their solution does not include $\mathrm{J}_{3}$ and $\mathrm{J}_{4}$ terms. According to Aksnes's numerical experiments comparing his second-order solution (Aksnes, 1970) with numerical integration, residuals of a few decimeters remain in position, most of which come from the third-order interaction among $\mathrm{J}_{2}, \mathrm{~J}_{3}$, and $\mathrm{J}_{4}$. On the other hand, the accuracy of current geodetic satellites equipped with retroreflectors for laser ranging will reach 3 to $5 \mathrm{~cm}$. Therefore, we can expect to obtain more accurate information on satellite motions by taking into account the third-order periodic perturbations.

In this paper, we consider only the zonal-harmonics perturbations of second, third, and fourth order. We chose a Keplerian motion as an intermediate orbit and adopted Hori's (1966) perturbation method. We assumed that the eccentricity of a geodetic satellite requiring highty

This work was supported in part by Grant NGR 09-015-002 from the National Aeronautics and Space Administration. 
accurate solutions is usualiy low; therefore, all quantities in the present solution are expanded into power series of the eccentricity, but the solution is obtained in closed form with respect to the inclination. Delaunay variables were selected as the canonical elements used to construct the new Hamiltonian and the determining functions eliminating periodic terms, which do not depend on the chosen canonical variables so long as Keplerian motion is adopted as an intermediate orbit. However, the final expressions of the periodic perturbations are given in $\ell+g, h$, e cos $g$, e $\sin g$, and $L$, which are not singular at zero eccentricity.

A11 literal calculations were carried out by means of the computer algebra program Smithsonian Package for Algebra and Symbolic Mathematics (SPASM) (Hal1 and Cherniack, 1969). Final results were checked analytically in various ways and were compared with the results of numerical integration. 


\section{OUTLINE OF THE METHOD OF SOLUTION}

Let us consider an artificial satellite orbiting in an axially symmetric gravitational field of the earth, whose force function $U$ is of the form

$$
U=\frac{\mu}{r}\left[1-\sum_{n=2}^{4}\left(\frac{a_{e}}{r}\right)^{n} J_{n} P_{n}(\sin \beta)\right],
$$

where $a_{e}$ is the equatorial radius of the earth, $r$ the radius vector of the satellite, and $\beta$ the declination. In this paper, the coefficient $\mathrm{J}_{2}$ is assumed to be a small quantity of the first order and $\mathrm{J}_{3}$ and $\mathrm{J}_{4}$ are of the second order.

To solve for the motion of the satellite under the force function (1), we adopted Hori's (1966) perturbation method, which utilizes Lie transformation; all formulas are canonically invariant, and the perturbations of any quantity are given by simple formulas and in explicit form. Because of the generality of Hori's method, we can choose any set of canonical variables. In the present paper, we use Delaunay variables as a canonical set for their simplicity, where $\ell$ is the mean anomaly, $g$ is the argument of perigee, $h$ is the longitude of the ascending node, and

$$
L=\sqrt{\mu a} ; \quad G=L \sqrt{1-e^{2}}, \quad H=G \cos i .
$$


The equations of motion are

$$
\frac{d}{d t}(L, G, H)=\frac{\partial F}{\partial(l, g, h)}, \quad \frac{d}{d t}(l, g, h)=-\frac{\partial F}{\partial(L, G, H)},
$$

where

$$
\begin{aligned}
& F=F_{0}+F_{1}+F_{2}, \\
& F_{0}=\frac{\mu^{2}}{2 L^{2}}, \\
& F_{1}=-\frac{\mu a_{e}^{2}}{r^{3}} J_{2} P_{2}(\sin \beta), \\
& F_{2}=-\frac{\mu a_{e}^{3}}{r^{4}} J_{3} P_{3}(\sin \beta)-\frac{\mu a_{e}^{4}}{r^{5}} J_{4} P_{4}(\sin \beta) .
\end{aligned}
$$

Under the assumption that the eccentricity is sma11, we expanded the disturbing functions $F_{1}$ and $\dot{F}_{2}$ into a power series of the eccentricity:

$$
\begin{aligned}
F_{1} & =\frac{\mu^{4} J_{2}}{L^{6}} \sum_{p=0}^{1} \sum_{k=-\infty}^{\infty} x_{k}^{2,2 p}(e) B_{2,2 p}(i) \cos (k \ell+2 p g), \\
F_{2} & =\frac{\mu^{5} J_{3}}{L^{8}} \sum_{p=0}^{1} \sum_{k=-\infty}^{\infty} x_{k}^{3,2 p+1} \text { (e) } B_{3,2 p+1}(i) \sin [k \ell+(2 p+1) g], \\
& +\frac{\mu^{6} J_{4}}{L^{10}} \sum_{p=0}^{2} \sum_{k=-\infty}^{\infty} x_{k}^{4,2 p}(e) B_{4,2 p}(i) \cos (k \ell+2 p g),
\end{aligned}
$$


where $x_{k}^{n, m}$ (e.) is a Hansen coefficient and

$$
\begin{aligned}
& B_{20}=-\frac{1}{4}\left(-2+3 \sin ^{2} i\right), \quad B_{22}=\frac{3}{4} \sin ^{2} i, \\
& B_{31}=-\frac{3}{8} \sin i\left(-4+5 \sin ^{2} i\right), \\
& B_{40}=-\frac{3}{8}\left(8-40 \sin ^{2} i+35 \sin ^{4} i\right), \\
& B_{42}=\frac{5}{16} \sin ^{2} i\left(-6+7 \sin ^{2} i\right),
\end{aligned}
$$

When the lowest powers of e and $\sin i$ of the coefficient of $\cos (k \ell+q g)$ in the trigonometric series in equations (4) are $\alpha$ and $\delta$, we have the following relations:

$$
\begin{aligned}
& \alpha=|k-q| \quad(\bmod .2), \\
& \delta=|q| \quad(\bmod , 2),
\end{aligned}
$$

which are called the d'Alembert characteristics.

The algorithm for deriving the new Hamiltonian $F^{*}$ (Hori, 1966) and the determining function $S$ eliminating short-period terms is

zeroth order:

$$
F_{0}^{*}=F_{0},
$$


Tors order:

$$
\begin{aligned}
& F_{1}^{*}=F_{1 s}, \\
& S_{1}=\frac{L^{\prime 3}}{\mu^{2}} \int F_{1 p} d \ell^{\prime},
\end{aligned}
$$

- second order:

$$
\begin{aligned}
& F_{2}^{*}=F_{2 s}+\frac{1}{2}\left\{F_{1}+F_{1}^{*}, S_{1}\right\}_{s}, \\
& S_{2}=\frac{L^{\prime 3}}{\mu^{3}} \int\left(F_{2 p}+\frac{1}{2}\left\{F_{1}+F_{1}^{*}, S_{1}\right\}_{p}\right) d \ell^{\prime},
\end{aligned}
$$

third order:

$$
\begin{aligned}
F_{3}^{*} & =\frac{1}{12}\left\{\left\{F_{1 p}, S_{1}\right\}, S_{1}\right\}_{S}+\frac{1}{2}\left\{F_{2}+F_{2}^{*}, s_{1}\right\}_{S}+\frac{1}{2}\left\{F_{1}+F_{1}^{*}, S_{2}\right\}_{S}, \\
S_{3} & =\frac{L^{\prime}}{\mu^{2}} \int\left(\frac{1}{12} \cdot\left\{F_{1 p}, S_{1}\right\}, S_{1}\right\}_{p}+\frac{1}{2}\left\{F_{2}+F_{2}^{*}, S_{1}\right\}_{p} \quad(5 d) \\
& \left.+\frac{1}{2}\left\{F_{1}+F_{1}^{*}, S_{2}\right\}_{p}\right) d l^{\prime},
\end{aligned}
$$

fourth order:

$$
\begin{aligned}
F_{4}^{*} & =\frac{1}{2}\left\{F_{1}+F_{1}^{*}, S_{3}\right\}_{S}+\frac{1}{2}\left\{F_{2}+F_{2}^{*}, S_{2}\right\}_{S}+\frac{1}{2}\left\{F_{3}+F_{3}^{*}, S_{1}\right\}_{S} \\
& +\frac{1}{12}\left\{\left\{F_{1 p}, S_{1}\right\}, S_{2}\right\}_{S}+\frac{1}{12}\left\{\left\{F_{1 p}, s_{2}\right\}, S_{1}\right\}_{S} \\
& +\frac{1}{12}\left\{\left\{F_{2 p}, S_{1}\right\}, S_{1}\right\}_{S},
\end{aligned}
$$

(be)

6 
where the braces represent roisson brackets and the subscripts $s$ and $p$. indicate the constant and periodic parts in $\ell^{\prime}$. It should be noted that any function of $L^{\prime}, G^{\prime}, H^{\prime}$, and $g^{\prime}$ can be added to $S$.

The algorithm for calculating the new Hamiltonian $F^{* *}$ and the determining function $S^{*}$ eliminating long-period terms is given by the following equations:

first order:

$$
F_{1}^{* *}=F_{1}^{*}
$$

second order:

$$
\begin{aligned}
& F_{2}^{* *}=F_{2 s}^{*}, \\
& S_{1}^{*}=-\left(\frac{\partial F_{1}^{*}}{\partial G^{\pi}}\right)^{-1} \int F_{2 p}^{*} d g^{\prime \prime},
\end{aligned}
$$

third order:

$$
\begin{aligned}
& F_{3}^{* *}=\frac{1}{2}\left\{F_{2}^{*}+F_{2}^{* *}, S_{1}^{*}\right\}_{S}, \\
& S_{2}^{*}=-\frac{1}{2}\left(\frac{\partial F_{1}^{*}}{\partial G^{\prime \prime}}\right)^{-1} \int\left\{F_{2}^{*}+F_{2}^{* *}, S_{1}^{*}\right\}_{p} d g^{\prime \prime},
\end{aligned}
$$

fourth order:

$$
\begin{aligned}
\mathrm{F}_{4}^{* *} & =\frac{1}{12}\left\{\left\{\mathrm{~F}_{2 \mathrm{p}}^{*}, \mathrm{~S}_{1}^{*}\right\}, \mathrm{S}_{1}^{*}\right\}_{\mathrm{S}}+\frac{1}{2}\left\{\mathrm{~F}_{3}^{*}+\mathrm{F}_{3}^{* *}, \mathrm{~S}_{1}^{*}\right\}_{\mathrm{S}} \\
& +\frac{1}{2}\left\{\mathrm{~F}_{2}^{*}+\mathrm{F}_{2}^{* *}, \mathrm{~S}_{2}^{*}\right\}_{\mathrm{S}},
\end{aligned}
$$

(eq. (6d) cont. on next page) 


$$
\begin{aligned}
S_{3}^{*} & =-\left(\frac{\partial F_{1}}{\partial G^{\prime \prime}}\right)^{-} /\left(\frac{1}{12}\left\{\left\{F_{2 p}^{*}, S_{1}^{*}\right\}, S_{1}^{*}\right\}_{p}+\frac{1}{2}\left\{F_{3}^{*}+F_{3}^{* *}, S_{1}^{*}\right\}_{p}\right. \\
& \left.+\frac{1}{2}\left\{F_{2}^{*}+F_{2}^{* *}, S_{2}^{*}\right\}_{p}\right) d g^{\prime \prime},
\end{aligned}
$$

where the subscripts $s$ and $p$ indicate the constant and periodic parts in $g^{\prime \prime}$. These algorithms are actually very simple, but calculating them by hand is laborious. Therefore, all computations were carried out by the computer program SPASM (HaIl and Cherniack, 1969). SPASM handles the operations in equations (5) and (6) easily, keeping rational fractions for coefficients.

A key operation in equations (5) and (6) is evaluating the Poisson bracket $\{A, B\}$ :

$$
\{A, B\}=\frac{\partial A}{\partial L} \frac{\partial B}{\partial l}-\frac{\partial A}{\partial l} \frac{\partial B}{\partial L}+\frac{\partial A}{\partial G} \frac{\partial B}{\partial g}-\frac{\partial A}{\partial g} \frac{\partial B}{\partial G}+\frac{\partial A}{\partial H} \frac{\partial B}{\partial h}-\frac{\partial A}{\partial h} \frac{\partial B}{\partial H} \quad \cdot
$$

In the present theory, as we seek to obtain a solution that is closed with respect to the inclination, the atomic variables in the computer a]gebra are $L, e, s=\sin i, c=\cos i$, and $\gamma=1-5 \cos ^{2} i$ ( $\gamma$ appears in the denominator of $S^{*}$ ). The derivatives with respect to $L, G$, and $H$ are

$$
\begin{aligned}
\frac{\partial}{\partial L} & =\left(\frac{\partial}{\partial L}\right)+\frac{1-e^{2}}{e L} \frac{\partial}{\partial e}, \\
\frac{\partial}{\partial G} & =\frac{1}{L \sqrt{1-e^{2}}}\left(-\frac{1-e^{2}}{e} \cdot \frac{\partial}{\partial e}+\frac{1-\sin ^{2} i}{\sin i} \frac{\partial}{\partial S}-\cos i \frac{\partial}{\partial c}\right. \\
& +10\left(1-\sin ^{2} \text { i) } \frac{\partial}{\partial \gamma}\right),
\end{aligned}
$$

(eq. (8) cont. on next page) 


$$
\frac{\partial}{\partial H}=-\frac{1}{L \sqrt{1-e^{2}}}\left(\frac{\cos i}{\sin i} \frac{\partial}{\partial s}+\frac{\partial}{\partial c}+10 \cos i \frac{\partial}{\partial \gamma}\right) \text {, }
$$

where the factor $\sqrt{1-e^{2}}$ wi 17 be replaced by a Taylor expansion in powers of $e$. If both $A$ and $B$ satisfy the d'Alembert charàcteristics, then even if these derivatives include the terms $1 / \mathrm{e}$ and $1 / \sin ^{\prime} i$, the Poisson bracket $\{A, B\}$ keeps the d'Atembert characteristics and does not have $1 / \mathrm{e}$ and $1 / \sin i$ in the expression. This serves as a good check on Titeral manipulation by a computer. In Deprit and Rom's (1970) theory, $\cos i=H / L \sqrt{i-e^{2}}$ is developed in power series of e. Therefore, their determining function $W$ apparently loses the d'Alembert characteristics with respect to the inclination. 'If $A$ and $B$ are expanded into power series of $e$ and truncated at $e^{n}$, the derivatives with respect to $L$ and $G$ are correct up to $e^{n-2}$; however, $\{A, B\}$ is correct up to $e^{n-1}$. In other words, with one operation of the Poisson bracket, only one degree in e (not two) is lost. Our program, which takes this fact into consideration, sàves a lot of computer time.

Complete to $\mathrm{J}_{2}^{3}$, the analytical solution must take into account all the following terms:

first order:

$$
\mathrm{J}_{2}, \quad \mathrm{~J}_{3} / \mathrm{J}_{2}, \quad \mathrm{~J}_{4} / \mathrm{J}_{2},
$$

second order:

$$
\begin{aligned}
& \mathrm{J}_{3}, \mathrm{~J}_{3} \mathrm{~J}_{4} / \mathrm{J}_{2}^{2}, \quad \mathrm{~J}_{2}^{2}, \\
& \left(\mathrm{~J}_{3} / \mathrm{J}_{2}\right)^{2}, \quad\left(\mathrm{~J}_{4} / \mathrm{J}_{2}\right)^{2},
\end{aligned}
$$


third order:

$$
\begin{aligned}
& \mathrm{J}_{2} \mathrm{~J}_{3}, \quad \mathrm{~J}_{3} \mathrm{~J}_{4}^{2} / \mathrm{J}_{2}^{3}, \quad\left(\mathrm{~J}_{3} / \mathrm{J}_{2}\right)^{3}, \quad \mathrm{~J}_{3} \mathrm{~J}_{4} / \mathrm{J}_{2}, \\
& \mathrm{~J}_{2}^{3}, \quad \mathrm{~J}_{2} \mathrm{~J}_{4}, \quad\left(\mathrm{~J}_{4} / \mathrm{J}_{2}\right)^{3}, \quad \mathrm{~J}_{3}^{2} \mathrm{~J}_{4} / \mathrm{J}_{2}^{3}, \\
& \mathrm{~J}_{3}^{2} / \mathrm{J}_{2} \quad, \quad \mathrm{~J}_{4}^{2} / \mathrm{J}_{2} \quad \text {. }
\end{aligned}
$$

Most of these terms arise from the interaction among $\mathrm{J}_{2}, \mathrm{~J}_{3}$, and $\mathrm{J}_{4}$.

Tables 1 and 2 list the numbers of terms involved in $S_{3}$ and $S_{3}^{*}$, respectively. The total number of terms with the factor $J_{2}^{3}$ in $S_{3}$ is 106 . On the other hand, Deprit and Rom's $W_{3}$ contains 192 terms up to $e^{4}$, partly because they chose $n=H / L$ as one of the arguments. Explicit expressions of $S, F^{*}, S^{*}$, and $F^{* *}$ are given in Appendix $A$.

Table 1. Numbers of terms in the determining function $\mathrm{S}_{3}$.

\begin{tabular}{crrrr}
\hline $\begin{array}{c}\text { Order of } \\
\text { eccentricity }\end{array}$ & $\mathrm{J}_{2}^{3}$ & $\mathrm{~J}_{2} \mathrm{~J}_{3}$ & $\mathrm{~J}_{2} \mathrm{~J}_{4}$ & Total \\
\hline $\mathrm{e}^{0}$ & 6 & 6 & 6 & 18 \\
$\mathrm{e}^{1}$ & 16 & 8 & 16 & 40 \\
$\mathrm{e}^{2}$ & 19 & 18 & 18 & 55 \\
$\mathrm{e}^{3}$ & 32 & 19 & 32 & 83 \\
$\mathrm{e}^{4}$ & $\underline{33}$ & $\underline{30}$ & $\underline{31}$ & $\underline{94}$ \\
Total & 106 & 81 & 103 & 290 \\
\hline
\end{tabular}


Table 2. Numbers of terms in the determining function $\mathrm{S}_{3}^{*}$.

\begin{tabular}{cccccccccccc}
\hline $\begin{array}{c}\text { Order of } \\
\text { eccentricity }\end{array}$ & $\mathrm{J}_{3} \mathrm{~J}_{4}^{2} / \mathrm{J}_{2}^{3}$ & $\left(\mathrm{~J}_{3} / \mathrm{J}_{2}\right)^{3}$ & $\mathrm{~J}_{3} \mathrm{~J}_{4} / \mathrm{J}_{2}$ & $\mathrm{~J}_{2} \mathrm{~J}_{3}$ & $\left(\mathrm{~J}_{4} / \mathrm{J}_{2}\right)^{3}$ & $\mathrm{~J}_{3}^{2} \mathrm{~J}_{4} / \mathrm{J}_{2}^{3}$ & $\mathrm{~J}_{4}^{2} / \mathrm{J}_{2}$ & $\mathrm{~J}_{3}^{2} / \mathrm{J}_{2}$ & $\mathrm{~J}_{2} \mathrm{~J}_{4}$ & $\mathrm{~J}_{2}^{3}$ & Tot \\
\hline $\mathrm{e}^{1}$ & 5 & 4 & 8 & 9 & -- & -- & -- & -- & -- & -- & 2 \\
$\mathrm{e}^{2}$ & -- & -- & -- & -- & 6 & 7 & 15 & 7 & 15 & 15 & 6 \\
$\mathrm{e}^{3}$ & $\underline{19}$ & $\underline{14}$ & $\underline{26}$ & $\underline{26}$ & $\underline{-}$ & $\underline{-}$ & $\underline{-}$ & $\underline{-}$ & $\underline{-}$ & $\underline{-}$ & $\frac{8}{7}$ \\
Total & 24 & 18 & 34 & 35 & 6 & 7 & 15 & 7 & 15 & 15 & 17 \\
\hline
\end{tabular}


When both short- and long-period terms have been eliminated, the new Hamiltonian $\mathrm{F}^{* *}$ contains no angular variables. The action variables $L^{\prime \prime}, G^{\prime \prime}$, and $H^{\prime \prime}$ are constant, and the angular variables $\ell^{\prime \prime}, g^{\prime \prime}$, and - $h^{\prime \prime}$ are expressed as follows:

$$
\begin{aligned}
& \ell^{\prime \prime}=-\frac{\partial}{\partial L^{\prime \prime}} F^{* *}+\ell_{0}^{\prime \prime}, \\
& g^{\prime \prime}=-\frac{\partial}{\partial G^{\prime \prime}} F^{* *}+g_{0}^{\prime \prime}, \\
& h^{\prime \prime}=-\frac{\partial}{\partial H^{\prime \prime}} F^{* *}+h_{0}^{\prime \prime}
\end{aligned}
$$

The generating functions $S$ and $S *$ determine a completely canonical transformation from the osculating elements ( $L, G, H, l, g$, and $h$ ) to the mean elements $\left(L^{\prime \prime}, G^{\prime \prime}, H^{\prime \prime}, l^{\prime \prime}, g^{\prime \prime}\right.$, and $\left.h^{\prime \prime}\right)$. The periodic perturbations of $\ell$ and $g$ have negative powers of the eccentricity as a factor, which reflects the fact that the perigee is not well determined when the eccentricity is smalt. If the eccentricity is smaller than $\left(-\mathrm{J}_{3} / 2 \mathrm{~J}_{2} \mathrm{a}\right) \sin i$, the argument of perigee librates and does not circulate (Kozai, 1966; Kinoshita and Hori, 1972; Hori, 1973). Because $\ell, g$, and e do not satisfy the d'Alembert characteristics with respect to the eccentricity, we selected $\ell+g, h$, e $\cos g$, e $\sin g, L$, and $H$ as the set of elements for calculating the ephemeris of a satelitite, as was also done by Deprit and Rom (1970). If $\varepsilon$ stands for one of these elements, the osculating element $\varepsilon$ is obtained from the following formulas through the third order of $j_{2}^{3}$ : 


$$
\begin{aligned}
\varepsilon & =\varepsilon^{\prime}+\left\{\varepsilon^{\prime}, S\right\}+\frac{1}{2}\left\{\left\{\varepsilon^{\prime}, S\right\}, S\right\}+\frac{1}{6}\left\{\left\{\left\{\varepsilon^{\prime}, S\right\}, S\right\}, S\right\}+O\left(J_{2}^{4}\right), \\
\varepsilon^{\prime} & =\varepsilon^{\prime \prime}+\left\{\varepsilon^{\prime \prime}, S^{*}\right\}+\frac{1}{2}\left\{\left[\varepsilon^{\prime \prime}, S^{*}\right\}, S^{*}\right\}+\frac{1}{6}\left\{\left\{\left\{\varepsilon^{\prime \prime}, S^{*}\right\}, S^{*}\right\}, S^{*}\right\} \\
& +o\left(J_{2}^{4}\right) .
\end{aligned}
$$

These expressions do not contain negative powers of the eccentricity. Even though neither $\ell+g$ nor $h$ satisfies the d'Alembert characteristics with respect to the inclination, the sum of $l+g+h$ does, thus providing another good check on the lengthy calculations. Explicit expressions of $\partial F^{* *} / \partial(L, G, H)$, short-period perturbations $\varepsilon-\varepsilon^{\prime}$, and Tong period perturbations $\varepsilon^{\prime}-\varepsilon^{\prime \prime}$ are given in Appendix $B$. 


\section{COMPUTATION OF POSITION AND VELOCITY}

Given the mean elements $a_{0}^{\prime \prime}, e_{0}^{\prime \prime}, i_{0}^{\prime \prime}, \ell_{0}^{\prime \prime}, g_{0}^{\prime \prime}$, and $h_{0}^{\prime \prime}$ at the initia] epoch, which are determined from observations, the position and velocity' of the satellite at an arbitrary epoch $t$ measured from the initial epoch can be calculated from the followirig procedures:

A. First we compute the secular perturbations from equations (9) plus $L^{\prime \prime}=\sqrt{a_{0}^{\prime \prime}}, G^{\prime \prime}=L^{\prime \prime} \sqrt{1-e_{0}^{\prime \prime 2}}$, and $H^{\prime \prime}=G^{\prime \prime} \cos i_{0}^{\prime \prime}$.

B. From equations (10), we compute the long- and short-period perturbations.

C. Now we have the osculating elements $\xi=e \cos g, \eta=e \sin g$, $\lambda=\ell+g, h, L$, and $H$. The method of calculating the position and velocity from these osculating elements is given in Deprit and Rom (1970).

Kepler's equation in the form

$$
\psi=\lambda+\xi \sin \psi-\eta \cos \psi
$$

is solved by iteration to obtain $\psi=E+g$, where $E$ is the eccentric anomaly. The eccentricity, the semimajor axis, and the inclination are computed from the relations

$$
e^{2}=\xi^{2}+n^{2}, \quad a=L^{2}, G=L \sqrt{1-e^{2}}, \quad \cos i=H / G
$$


The position and the velocity in the nodal frame of reference are given by

$$
\begin{aligned}
& X=a\left[\cos \psi-\xi+\frac{n}{1+\sqrt{1-e^{2}}}(\xi \sin \psi-n \cos \psi)\right], \\
& Y=a\left[\sin \psi-n-\frac{\xi}{1+\sqrt{1-e^{2}}}(\xi \sin \psi-n \cos \psi)\right], \\
& \dot{X}=-\frac{1}{G}\left(\xi+\frac{Y}{r}\right), \\
& \dot{Y}=\frac{1}{G}\left(n+\frac{X}{r}\right),
\end{aligned}
$$

where $r=\sqrt{x^{2}+y^{2}}$. Finally, we have the position and velocity in the inertial frame:

$$
\begin{aligned}
& x=X \cos h-Y \cos i \sin h, \\
& y=X \sin h+Y \cos i \cos \dot{h}, \\
& z=Y \sin i, \\
& \dot{x}=\dot{X} \cos h-\dot{Y} \cos i \sin h, \\
& \dot{y}=\dot{X} \sin h+\dot{y} \cos i \cos h, \\
& \dot{z}=\dot{y} \sin i .
\end{aligned}
$$


The semimajor axis is usually obtained from the mean motion of the mean anomaly. When the eccentricity is small, the mean anomaly is not well determined, because it is strongly correlated with the argument of perigee. On the other hand, even if the eccentricity is very small, the mean argument of latitude $\ell+\mathrm{g}$ is well determined and does not suffer so much from the inaccuracy of the long-period perturbations. The long-period perturbation of $\ell+g$ is expressed as

$$
{ }^{\Delta} \text { long }(l+g)=e A_{1}(e, i) \cos g+e^{2} A_{2}(e, i) \sin 2 g+o\left(e^{3}\right),
$$

which satisfies the d'Alembert characteristics; this expression is also small because the amplitudes of $\cos g$ and $\sin 2 g$ have factors of $e$ and $e^{2}$, respectively. On the other hand, since the eccentricity appears in the denominator in the long-period perturbation of the mean anomaly, the semimajor axis is determined with better accuracy from the mean motion of $\ell+g$ than from the mean motion of $\ell$. 


\section{ANALYTICAL AND NUMERICAL CHECK .}

Hori (1970a) and Yuasa (1971) showed that both Hori's and von Zeipel's perturbation theories give the same canonical transformation, . together with the same new Hamiltonian, up to the third order. This allows us to compare the present solution, based on Hori's theory, with Kozai's (1962) solution, based on von Zeipel's theory. Our $\mathrm{F}_{3}^{* *}$ completely agrees with Kozai's $F_{3}^{* *}$. Although Hori (1966) gave a relation between the determining functions of his and von Zeipel's theories, we have not compared these functions, because of the complexity of Kozai's expression of $\mathrm{S}_{2}$ and the tediousness of the calculations. Instead, we compared our $S_{2}$ with that derived later by Hori (1970b). In determining $S$, we have an ambiguity and can add an arbitrary function of $L, G, H$, and $g$ to $S$, giving us $\bar{S}$. In the present theory, the disturbing function is expanded into a Fourier series with arguments of $\ell$ and $g$; therefore, it is natural to determine $S$ in such a manner that its mean value with respect to the mean anomaly is zero. On the other hand, the mean value of Hori's $S$ is not zero. The relation between $S$ and $\vec{S}$, both of which are determining functions eliminating short-period terms in the Hamiltonian, is

$$
\begin{aligned}
& \bar{s}_{1}=s_{1}+f_{1}, \\
& \bar{s}_{2}=s_{2}+\frac{1}{2}\left\{s_{1}, f_{1}\right\}+f_{2},
\end{aligned}
$$


where $f_{1}$ and $f_{2}$ are arbitrary functions of $L, G, H$, and $g$. Then the relation between $S^{*}$ and $\bar{S}^{*}$, which are determining functions el iminating long-period terms, is

$$
\begin{aligned}
& \bar{s}_{1}^{*}=S_{1}^{*}-f_{1}, \\
& \bar{s}_{2}^{*}=s_{2}^{*}+\frac{1}{2}\left\{s_{1}^{*}, f_{1}\right\}-f_{2} .
\end{aligned}
$$

Even if the functional forms of these determining functions are different, the composite canonical transformation of $\left(S, S^{*}\right)$ is identical to that of $\left(\bar{S}, \cdot \bar{S}^{*}\right)$. The second-order determining function $S_{2}$ of the present : theory and that derived by Hori are found to satisfy relation (15).

It is of interest to compare the present solution with that due to Deprit and Rom (197.0), in which $\mathrm{J}_{3}$ and $\mathrm{J}_{4}$ are zeros. Their solution was obtained by their own perturbation method, which, like Hori's, is based on Lie transformation. Campbell and Jefferys (1970) showed that the perturbation theories of Hori and Deprit are equivalent and derived explicjt relations between the determining functions for the two:

$$
\begin{aligned}
& w_{1}=-s_{1}, \\
& w_{2}=-2 s_{2}, \\
& w_{3}=-6 s_{3}-\left\{s_{1}, s_{2}\right\},
\end{aligned}
$$


where $W_{n}$ are the determining functions in Deprit's theory. Using these relations, we compared our solution with Deprit and Rom's, which also serves as an independent check. The few disagreements, we found with their terms all seemed to be typographical errors. Kutuzov . (1975) also discovered some discrepancies between his solution, obtained. by computer algebra, and theirs.

As an internal consistency check, we wanted to make sure that a small divisor $\left(1-5 \cos ^{2} i\right)$ disappeared for the even-order harmonics when the geopotential was equal to that in Vinti (1959) $\left[J_{2 n}=(-1)^{n+1} J_{2}^{n}\right.$, $n \geq 2$ ]. In checking the third order, we had to add long-period perturbations arising from $\mathrm{J}_{6}$ and $\mathrm{J}_{8}$.

Finally, the present solution was compared with the results obtained from numerical integration. A Taylor-type integrator was adopted, in which the positions and velocities are expanded into a power series of time and the coefficients of the series are determined by recurrent formulas (Rabe, 1961; Deprit and Zahar, 1966). The order of the power - series and the step size of time were chosen so as to maintain about 12 significant figures in the integral of energy; the integration step is roughly one-fifth of the convergence radius $i$ of the two-body problem, $\tau=(1 / n)\left\{\ln \left[(1 / e)+\sqrt{\left(1 / e^{2}\right)-1}\right]-\sqrt{1-e^{2}}\right\}$, when the degree of the Taylor series is 16 . The numerical calculations were carried out by a CDC 6400 computer in double precision in order to avoid round-off errors. It takes about $0.7 \mathrm{sec}$ to evaluate the series of the present theory and about $0.3 \mathrm{sec}$ to integrate the equations of motion for one step. 
The initial conditions were computed from the present theory from a set of mean elements $a^{\prime \prime}, e^{\prime \prime}, i^{\prime \prime}, l^{\prime \prime}, g^{\prime \prime}$, and $h^{\prime \prime}$. It should be noted that the mean motion of the mean anomaly of the integrated orbit is expected to be different from the computed mean motion by an order of $\mathrm{J}_{2}^{4}$ because the accuracy of the periodic terms is of the order of $3_{2}^{3}$. This discrepancy can be avoided by adjusting the semimajor axis so as to remove the secular term in the residuals of the mean anomaly.

Such comparisons were done for the artificial satellites Geos 3, Starlette, Lageos, and Anna 1B. The results for Starlette are plotted in Figures 1 and 2. Figure 1 shows the in-track, along-track, and acrosstrack errors over two revolutions. The deviations, less than $2 \times 10^{-4} \mathrm{~m}$, are totally negligible. Figure 2 plots these same errors over about 600 revolutions. The deviations along and across the track are totally insignificant, but the in-track error has a secular trend that seems to be proportional to the square of time. The error first increases, then decreases, and finally vanishes at about $t=22$ days, at which time the semimajor axis was adjusted. This secular trend can be explained by the accumulation of truncation errors, which are caused by replacing infinitesimal operations with finite operations. The round-off errors do not accumulate significantiy, because our calculations were carried out in double precision, which amounts to an accuracy of about 30 significant figures in decimal notation. The accumulation error due to discretization (Kinoshita, 1968) is

$$
\Delta r_{\text {jn-track }} \propto a \ell^{2} h^{p},
$$



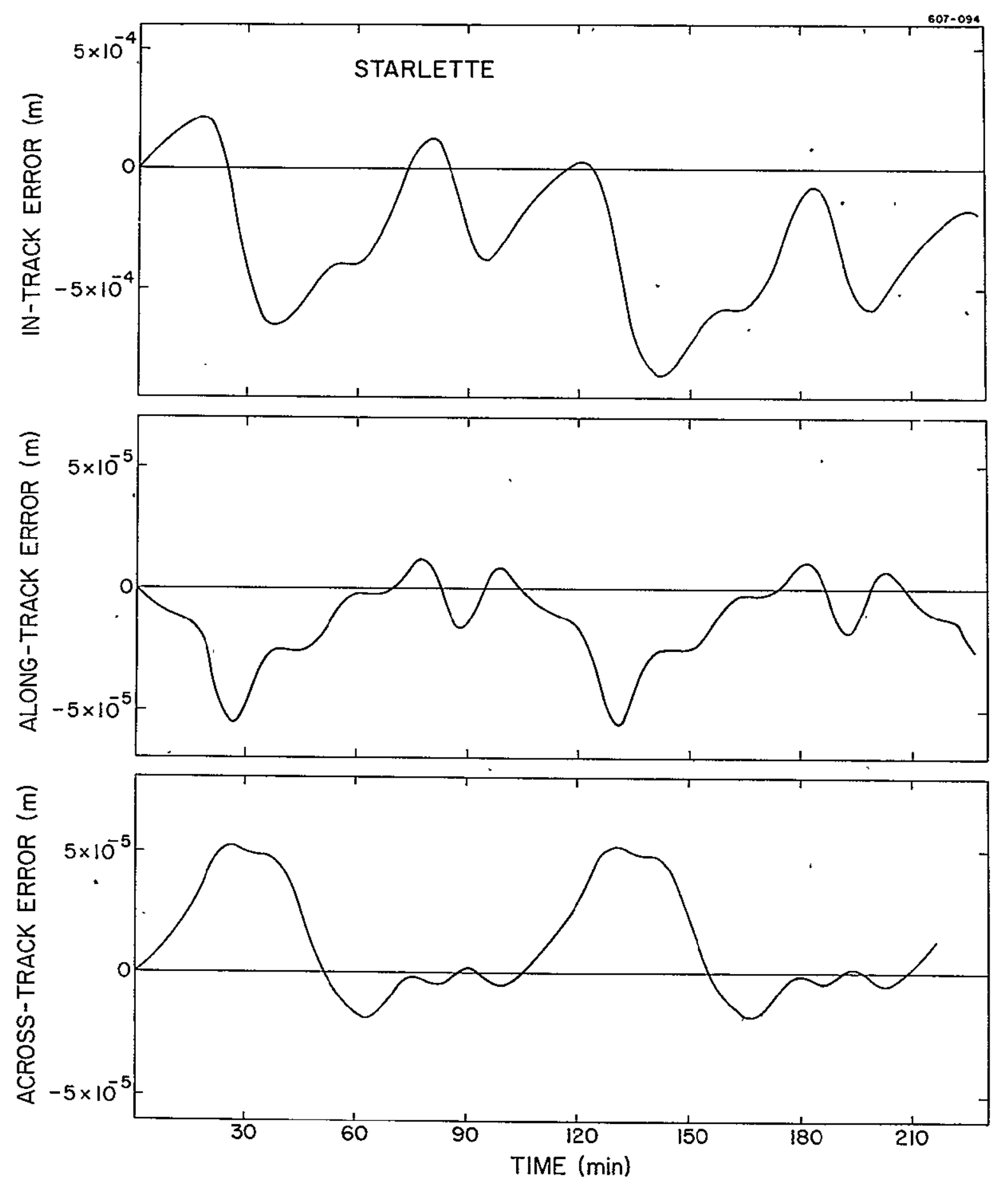

Figure 1. In-track, along-track, and across-track prediction errors of the third-order solution for Starlette plotted over two revolutions: $\mathrm{J}_{2}=1.082 \times 10^{-3}, \mathrm{~J}_{3}=-2.54 \times 10^{-6}, \mathrm{~J}_{4}=-1.619 \times$ $10^{-6}, a^{\prime \prime}=7.335 \times 10^{6} \mathrm{~m}, \mathrm{e}^{\prime \prime}=0.020636$, $i^{\prime \prime}=49.8223$, \&"0 $350: 23968, g_{0}^{\prime \prime}=82: 7702, h^{\prime \prime}=125.0266, \mathrm{P}=104.2 \mathrm{~min}$. 

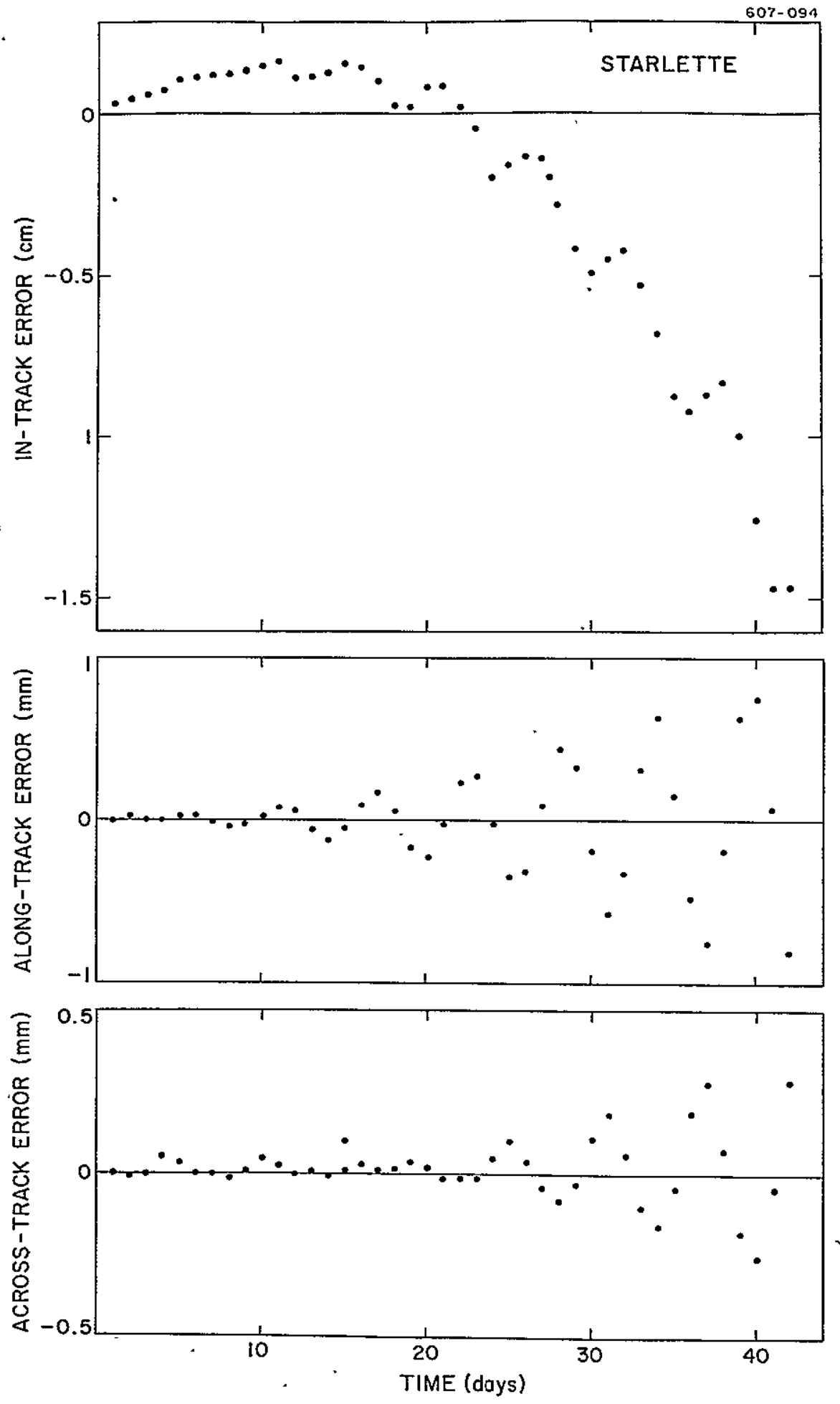

Figure 2. The same as Figure 1 plotted over about 600 revolutions. 
where $a$ is the semimajor axis, $l$ the mean anomaly, $h$ the step size, and $\mathrm{p}$ the degree of the Taylor expansion. About $1 \mathrm{~cm}$ of the in-track error at $t=40$ days $(1=3600$ radians $)$ is obtained from using equation (18) with $a=7.335 \times 10^{6} \mathrm{~m}$ and $\mathrm{h}=600 \mathrm{sec}=1 / 10$ radians, the order of the error agreeing with that of the numerica' experiment. . We can avoid discretization errors by employing a much more accurate integrator, but it seems to be an unnecessary use of computer time. The comparisons for Geos 3 and Lageos gave roughly the same results that we got for Starlette.

The errors for Anna $1 B$ are plotted in Figure 3, while Deprit and Rom's (1970) results for Anna 1B are reproduced in Figure 4. Even though the time span of our test is shorter than Deprit and Rom's, a comparison of Figures 3 and 4 indicates that our solution gives better predictions.

We are now confident that the solution presented herein can predict the position of a small-eccentricity geodetic satellite with an accuracy of better than $1 \mathrm{~cm}$ over 1 month. 

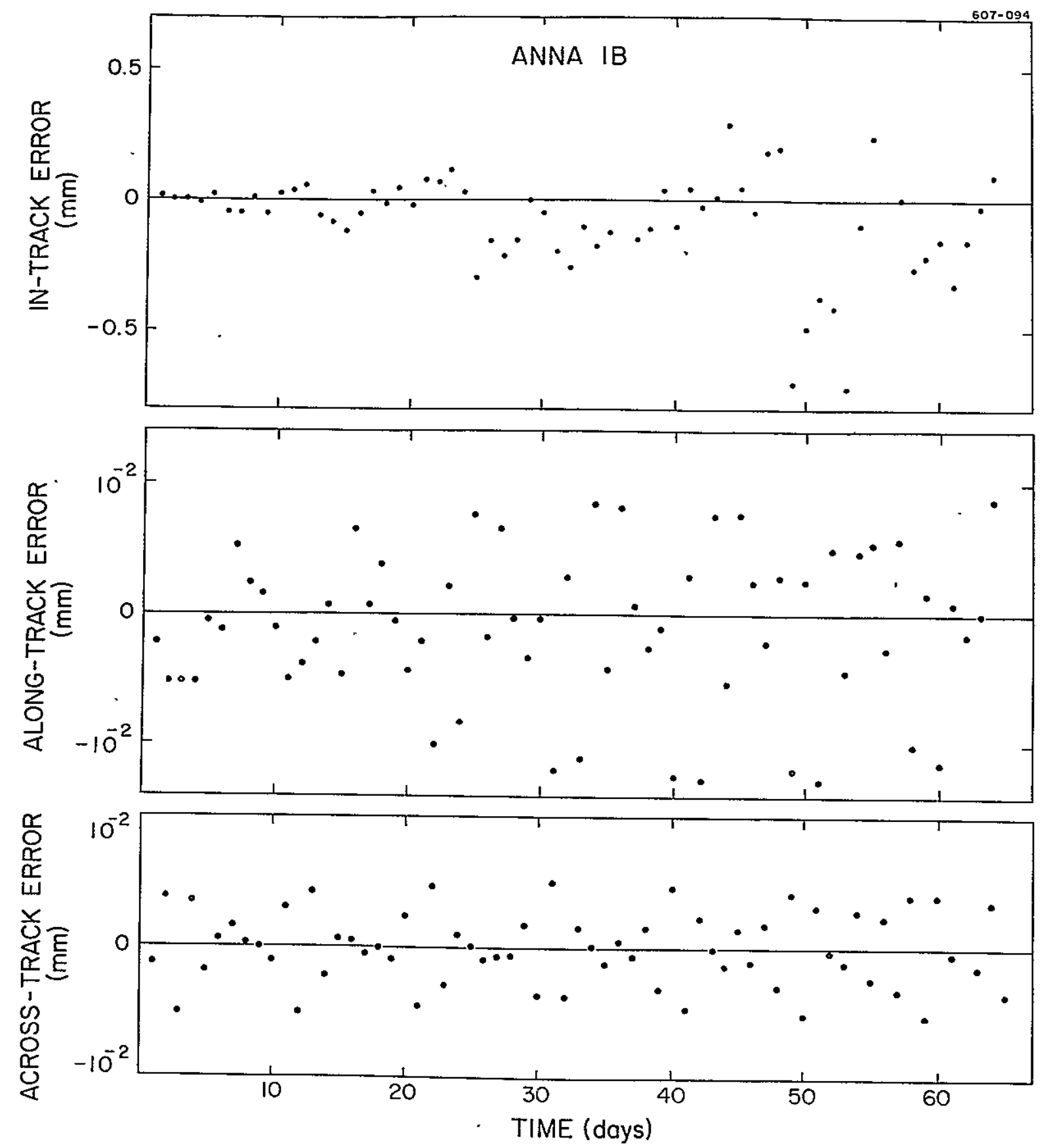

Figure 3. In-track, along-track, and across-track prediction errors of the third-order solution for Anna IB plotted over 860 revolutions: $\mathrm{J}_{2}=1.082 \times 10^{-3}, \mathrm{~J}_{3}=0, \mathrm{~J}_{4}=0, \mathrm{a}^{\prime \prime}=7.4738 \times 10^{6} \mathrm{~m}$, $e^{\prime \prime}=0.006711, i^{\prime \prime}=50^{\circ} .14768, \ell_{0}^{\prime \prime}=307^{\circ} .164, g_{0}^{\prime \prime}=198.303$, $\therefore$ $h_{0}^{\prime \prime}=54: 41, P=107.15 \mathrm{~min}$. 

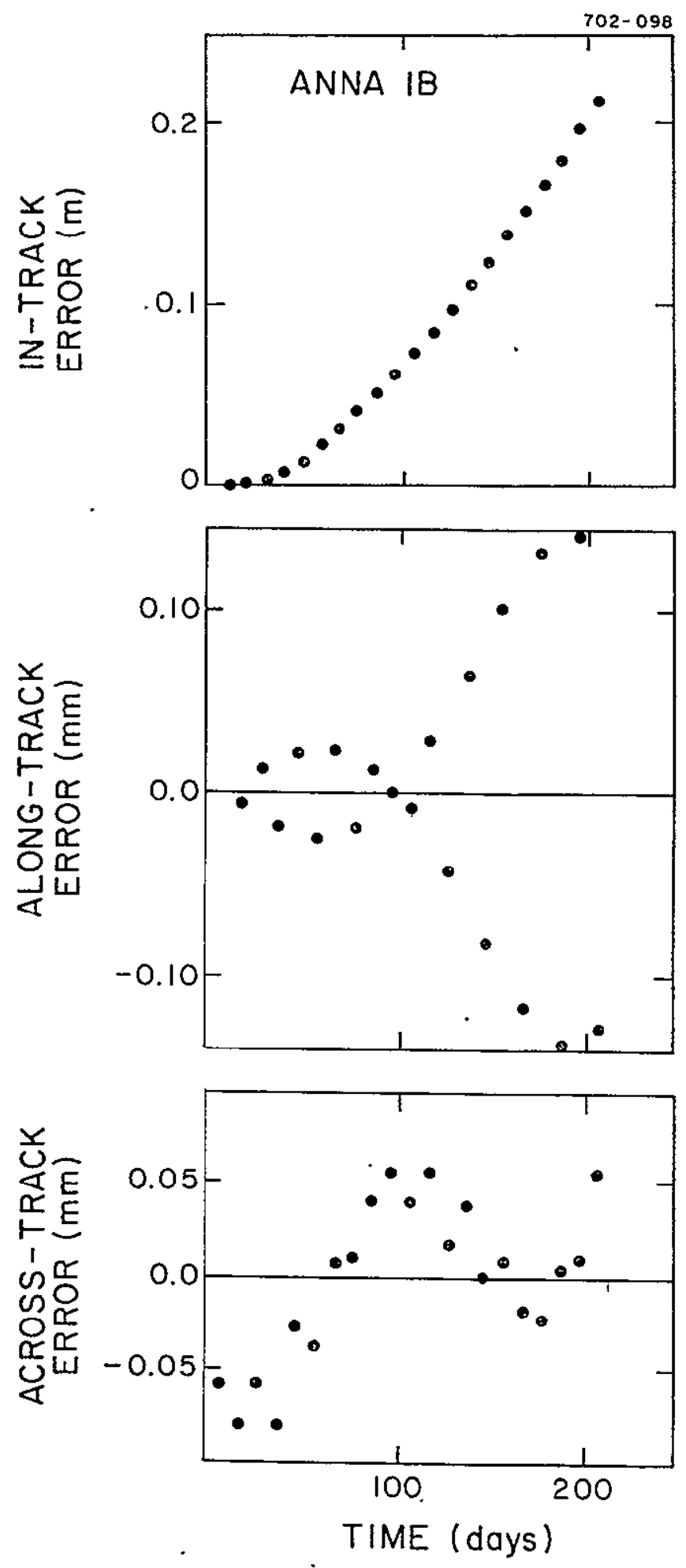

Figure 4. The same as Figure 3 plotted over about 2700 revolutions (from Deprit and Rom, 1970). 


\section{ACKNOWLEDGMENTS}

The author would like to acknowledge the continuing encouragement of Dr. E. M. Gaposchkin and expresses his appreciation to Dr. K. Aksnes · for valuable discussions. The author is also grateful to $\mathrm{Mr}$. J. R. Cherniack and $\mathrm{Mr}$. R. T. Poland for their occasional patience in explaining the mysteries of SPASM. 


\section{REFERENCES}

AKSNES, K.

1970. A second-order artificial satellite theory based on an inter- . mediate orbit. Astron. Journ., voi. 75, pp. 1066-1076.

CAMPBELL, A., and JEFFERYS, W. H.

1970. Equivalence of the perturbation theories of Hori and Deprit. Celest. Mech., vo1. 2, pp. 467-473.

DEPRIT, A., and ROM; A.

1970. The main problem of artificial satellite theory for small and moderate eccentricities. Celest. Mech., vol. 2, pp. 166-206.

DEPRIT, A.; and ZAHAR, R.

1966. Numerical integration of an orbit and its concomitant variations by recurrent power series. Zeit. Angew. Math. Phys., vol. 17 , pp. $426-430$.

HALL, N. M., and CHERNIACK, J. R.

1969. Smithsonian Package for Algebra and Symbolic Mathematics. Smithsonian Astrophys. Obs. Spec. Rep. No. 291, 49 pp.

ḢRI, G.

1966. Theory of general perturbations with unspecified canonical variables. Publ. Astron. Soc. Japan, vol. 18, pp. 287-296.

1970a. Comparison of two perturbation theories based on canonical transformations. Publ. Astron. Soc. Japan, vo1. 22, pp. 191-198. 
HORI, G.

1970b. Unpublished (paper read by B. Garfinkel at the International Astronomical Union General Assembly, Commission No. 7, Brighton, England, August).

1973. Theory of general perturbations. In Recent Advances in Dynamical Astronomy, ed. by B. D. Tapley, and V. Szebehely, D. Reide] PubT. Co., Dordrecht-Hol land, pp. 231-249.

HORI, G., and KOZAI, Y.

1975. Analytical theories of the motion of artificial satellites. In Satellite Dynamics, ed. by G. E. 0. Giacaglia, SpringerVerlag, Berlin, pp. 1-15.

KINOSHITA, H.

1968. Propagation of the discretization error in the two-body problem. Publ. Astron. Soc. Japan, vol. 20, pp. 1-23.

KINOSHITA, H., and HORI, G.

1972. On the stationaryit solutions for problems of artificial satel1ites. Tokyo Astron. Obs. Rep., vo1. 16, pp. 230-240.

KOZAI, Y.

1962. Second-order solution of artificial satellite theory without air drag. Astron. Journ., vol. 67, pp. 446-461.

1966. The earth gravitational potential derived from satellite motion. Space Sci. Rev., vol. 5, pp. 818-879.

KUTUZOV, A. L.

1975. Application of symbolic computer operations for solving the main problem in the theory of satellite motion. Soviet Astron. Lett., vol. 1, pp. 42-44. 
1961. Determination and survey of periodic Trojan orbits in the restricted problem of three bodies. Astron. Journ., vol. 66, pp. 500-513.

VINTI, J. P.

1959. New method of solution for unretarded satellite orbits. Journ. Res. Nat. Bur. Standards, vol. 63B, pp. 105-116.

YUASA, M.

- 1971. The comparison of Hori's perturbation theory and von Zeipel's theory. Publ. Astron. Soc. Japan, vol. 23, pp. 399-403. 
APPENDIX A

EXPRESSIONS OF $\mathrm{S}, \mathrm{F}^{*}, \mathrm{~S}^{\star}$, AND $\mathrm{F}^{\star \star}$

Some of the expressions herein are reproduced directly from computer printout, whose format differs from the type written equations. In the printout, the following notation is used:

$$
\begin{aligned}
& A(2)=J_{2}, \quad S I=\sin i, \quad E=e, \\
& A(3)=J_{3}, \quad C I=\cos 1, \quad S G=g, \\
& A(4)=J_{4}, \quad I N G=1-5 \cos ^{2} i, \quad M=2 .
\end{aligned}
$$

A.1 Expressions of $S$

$$
S=s_{1}\left(J_{2}\right)+s_{2}\left(J_{3}\right)+s_{2}\left(J_{4}\right)+s_{2}\left(J_{2}^{2}\right)+s_{3}\left(J_{2}^{3}\right)+s_{3}\left(J_{2} J_{3}\right)+s_{3}\left(J_{2} J_{4}\right)
$$$$
S_{1}\left(J_{2}\right)=\frac{J_{2}}{6 G^{3}}\left\{-\frac{3}{2}\left(I-3 c^{2}\right)(f-2+e \sin f)+\frac{3}{4}[3 e \sin (f+2 g)+3 \sin 2(f+g)+e \sin (3 f+2 g)]\right\}
$$$$
+\frac{J_{2}}{8 G^{3}} \frac{s^{2} e^{2}(1+2 n)}{(1+n)^{2}} \sin 2 g
$$

$$
S_{2}\left(J_{3}\right)=-\frac{J_{3}}{96 G^{5}} s\left\{3 ( l - 5 c ^ { 2 } ) \left[12 e(f-l) \sin g+3 e^{2} \cos (f-g)-6\left(2+e^{2}\right) \cos (f+g)-6 e \cos (2 f+g):\right.\right.
$$$$
\left.-e^{2} \cos (3 f+g)\right]+s^{2}\left[15 e^{2} \cos (f+3 g)+30 e \cos (2 f+3 g)+10\left(2+e^{2}\right) \cos 3(f+g)+15 e \cos (4 f+3 g)\right.
$$$$
\left.\left.+3 e^{2} \cos (5 f+3 g)\right]\right\}+\frac{J_{3}}{96 G^{5}}\left[6\left(1-5 c^{2}\right) \frac{\operatorname{se}\left(5+5 n+2 n^{2}\right)}{1+n} \cos g-\frac{s^{3} e^{3}\left(3+9 n+8 n^{2}\right)}{(1+n)^{3}} \cos 3 g\right]
$$

$$
\begin{aligned}
\mathrm{S}_{2}\left(\mathrm{~J}_{4}\right)= & \frac{\mathrm{J}_{4}}{512 \mathrm{G}^{7}}\left\{-2\left(3-30 \mathrm{c}^{2}+35 \mathrm{c}^{4}\right)\left[6\left(2+3 \mathrm{e}^{2}\right)(f-l)+9 e\left(4+\mathrm{e}^{2}\right) \cdot \sin f+9 e^{2} \sin 2 f+\mathrm{e}^{3} \sin 3 f\right]\right. \\
& +2 \mathrm{~s}^{2}\left(1-7 \mathrm{c}^{2}\right)\left[60 \mathrm{e}^{2}(f-2) \cos 2 g+10 \mathrm{e}^{3} \sin (f-2 g)+30 e\left(4+e^{2}\right) \sin (f+2 g)+20\left(2+3 e^{2}\right) \sin 2(f+g)\right. \\
& \left.+10 e\left(4+e^{2}\right) \sin (3 f+2 g)+15 e^{2} \sin (4 f+2 g)+2 e^{3} \sin (5 f+2 g)\right]-s^{4}\left[35 e^{3} \sin (f+4 g)\right. \\
& +105 e^{2} \sin (2 f+4 g)+35 e\left(4+e^{2}\right) \sin (3 f+4 g)+35\left(2+3 e^{2}\right) \sin 4(f+g)+21 e\left(4+e^{2}\right) \sin (5 f+4 g) \\
& \left.\left.+35 e^{2} \sin (6 f+4 g)+5 e^{3} \sin (7 f+4 g)\right]\right\}+\frac{J_{4}}{512 g^{7}}\left[\frac{2 s^{2}\left(1-7 c^{2}\right) e^{2}\left(77+154 n+10 n^{2}+28 n^{3}\right)}{(1+n)^{2}} \sin 2 g\right. \\
& -\frac{\left.s^{4} e^{4}\left(5+20 n+29 n^{2}+16 n^{3}\right) \sin 4 g\right]}{(1+n)^{4}}
\end{aligned}
$$

where $c=\cos i, s=\sin i, \eta=\sqrt{1-e^{2}}$. 


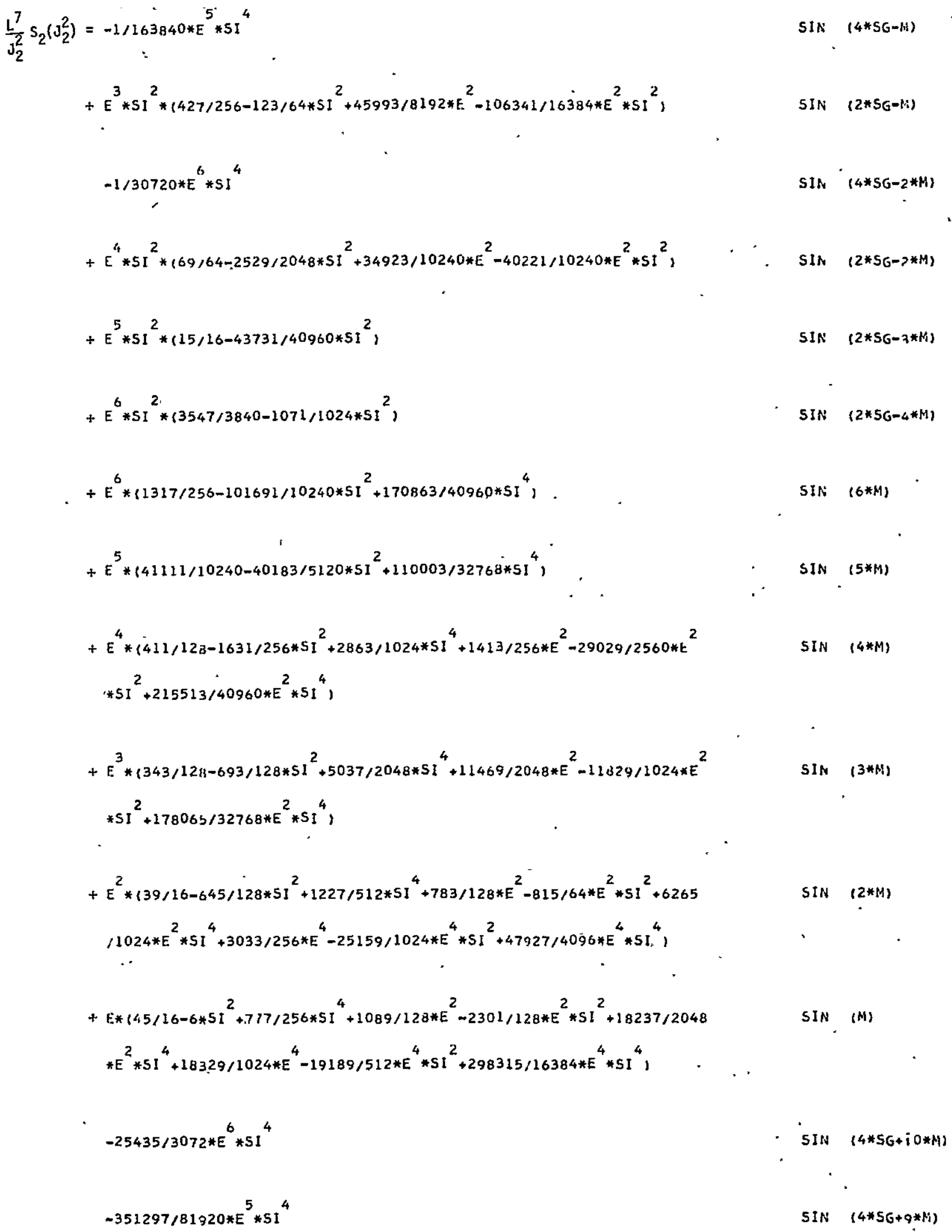




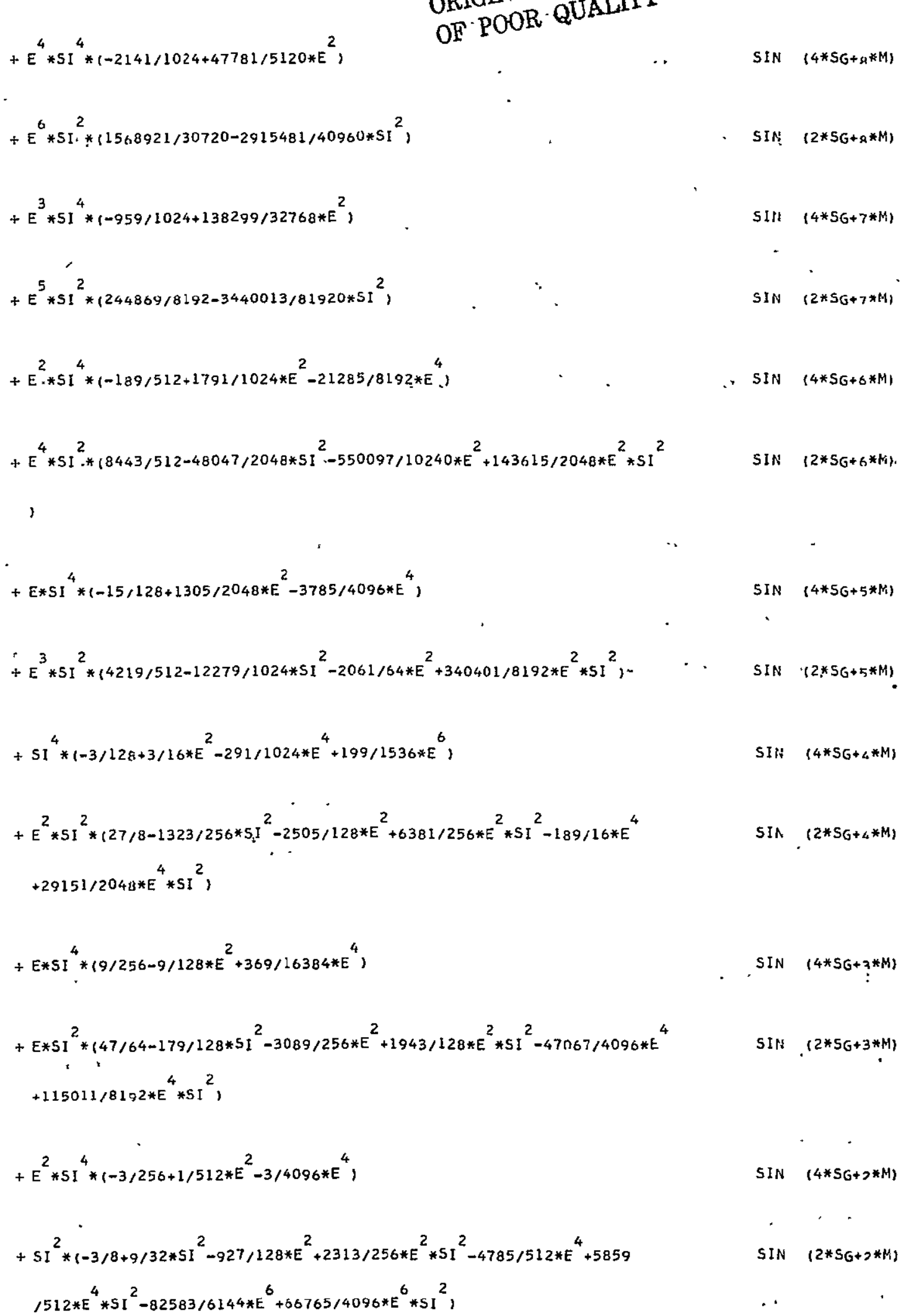




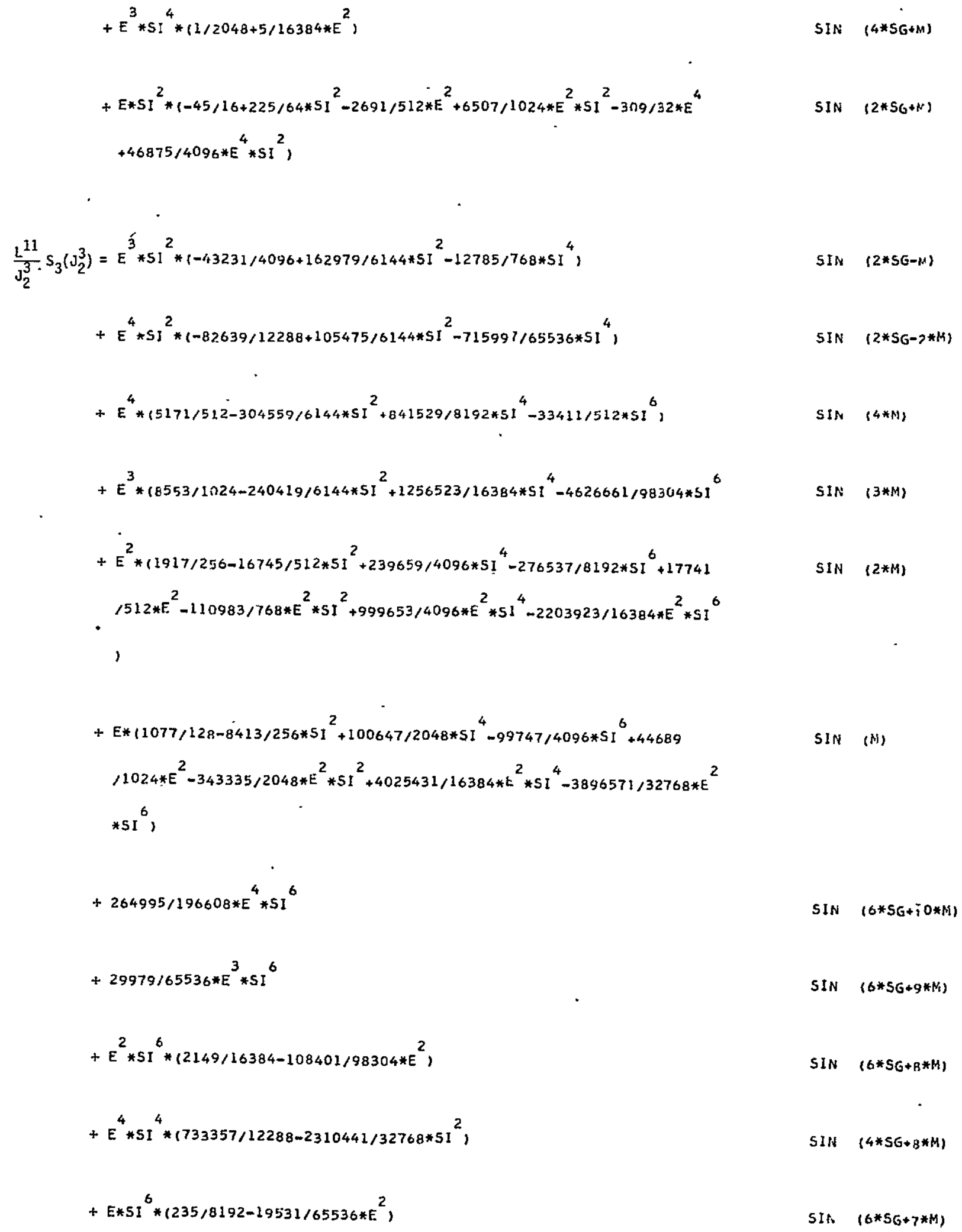


$+E^{3} * S^{4} *\left(2870799 / 114688-6818593 / 229376 * S^{2} I^{2}\right)$ OF POOR QUALITY

ORIGINAL PAGE IS

$\operatorname{SIN}(4 * 5 G+7 * M)$

$+\mathrm{SI}^{\circ} *\left(15 / 4096-63 / 1024 * \mathrm{E}^{2}+14463 / 65536 * \mathrm{E}^{4}\right)$

$\operatorname{SIN}(6 * S G+\alpha * M)$

$+E^{2} * S I^{4} *\left(18517 / 2048-44301 / 4096 * S I^{2}-282515 / 6144 * E^{2}+105793 / 2048 * E^{2}\right.$

$\sin (4 * S G+n * M)$ $* 5 I^{2}+$

$+E^{4} * S I^{2} *\left(397793 / 4096-5639543 / 24576 * 5 I^{2}+26702183 / 196608 * S I^{4}\right)$

$\operatorname{SIN}(2 * S G+6 * M)$

$+E * S I^{6} *\left(-61 / 8192+2731 / 65536 * E^{2}\right)$

$\sin (6 * 5 G+5 * 11)$

$+E * S I^{4} *\left(25907 / 10240-12537 / 4096 * S I^{2}-1572423 / 81920 * E^{2}+705243 / 32768 * E^{2} \quad S I A \quad(4 * 5 G+5 * M)\right.$ $* 5^{2}{ }^{2}$

$+E^{3} * S I^{2} *\left(1015027 / 20480-7160317 / 61440 * S I^{2}+3365033 / 49152 * S I^{4}\right.$,

$\operatorname{SIN}(2 * S G+5 * M)$

$\therefore 2 E^{6} * 577716384-901 / 98304 * E^{2}$

$\sin ^{2}(6 * S G+4 * M)$

$+S I^{4} *\left(105 / 256-519 / 1024 * 5 I^{2}-14409 / 2048 * E^{2}+4041 / 512 * E^{2} * 5 I^{2}-52611 l^{\prime} \quad 5 I N \quad(4 * S G+4 * M)\right.$ $12048 * E^{4}+122895 / 4096 * E^{4} * 5 I^{2}$,

$+E^{2} * S^{2} *\left(22871 / 1024-107261 / 2048 * 5 I^{2}+501057 / 16384 * S I^{4}-165433 / 6144 * E^{2} \quad\right.$ SIN $\quad(2 * S G+4 * M)$ $+383039 / 6144 * E^{2} * S^{2} I^{2}-1238799 / 32768 * E^{2} * 5 I^{4}$,

$-63 / 65536 * E^{3} * S 1^{6}$

$\operatorname{SIN}(6 * S G+3 * M)$

$+E * S I^{4} *\left(-11201 / 6144+8411 / 4096 * S I^{2}-457999 / 49152 * E^{2}+359975 / 3276 B * E^{2}\right.$

$\operatorname{SIN}(4 * 5 G+3 * M)$ $* S I^{2}$,

$+E * 5 I^{2} *\left(4069 / 512-28841 / 1536 * 5 I^{2}+67621 / 6144 * 5 I^{4}-70799 / 4096 * E^{2}\right.$

$\sin (2 * 5 G+2 * N)$ ${ }^{2} * I^{2}-1937753 / 98304 * E^{2} * S I^{4}$, 


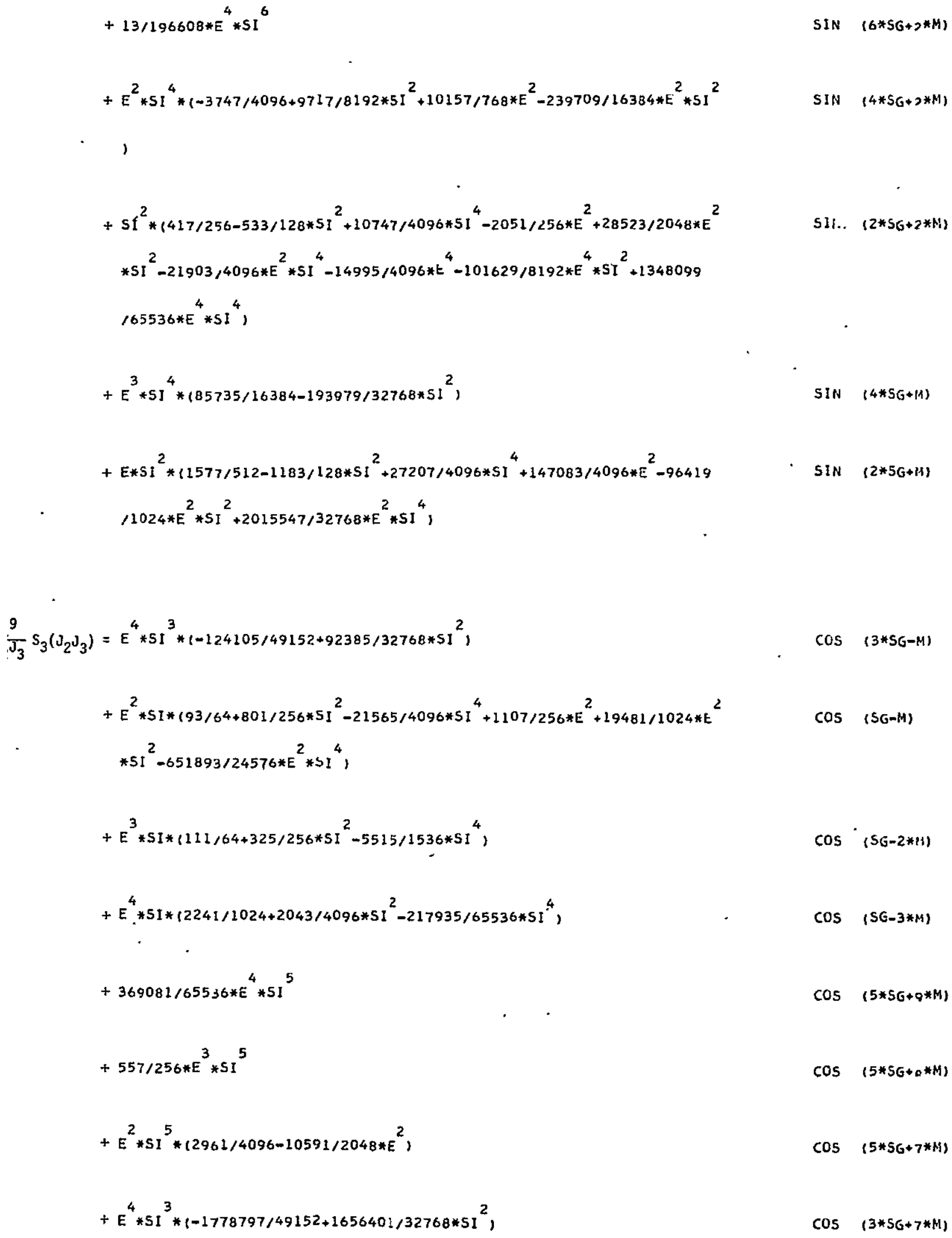




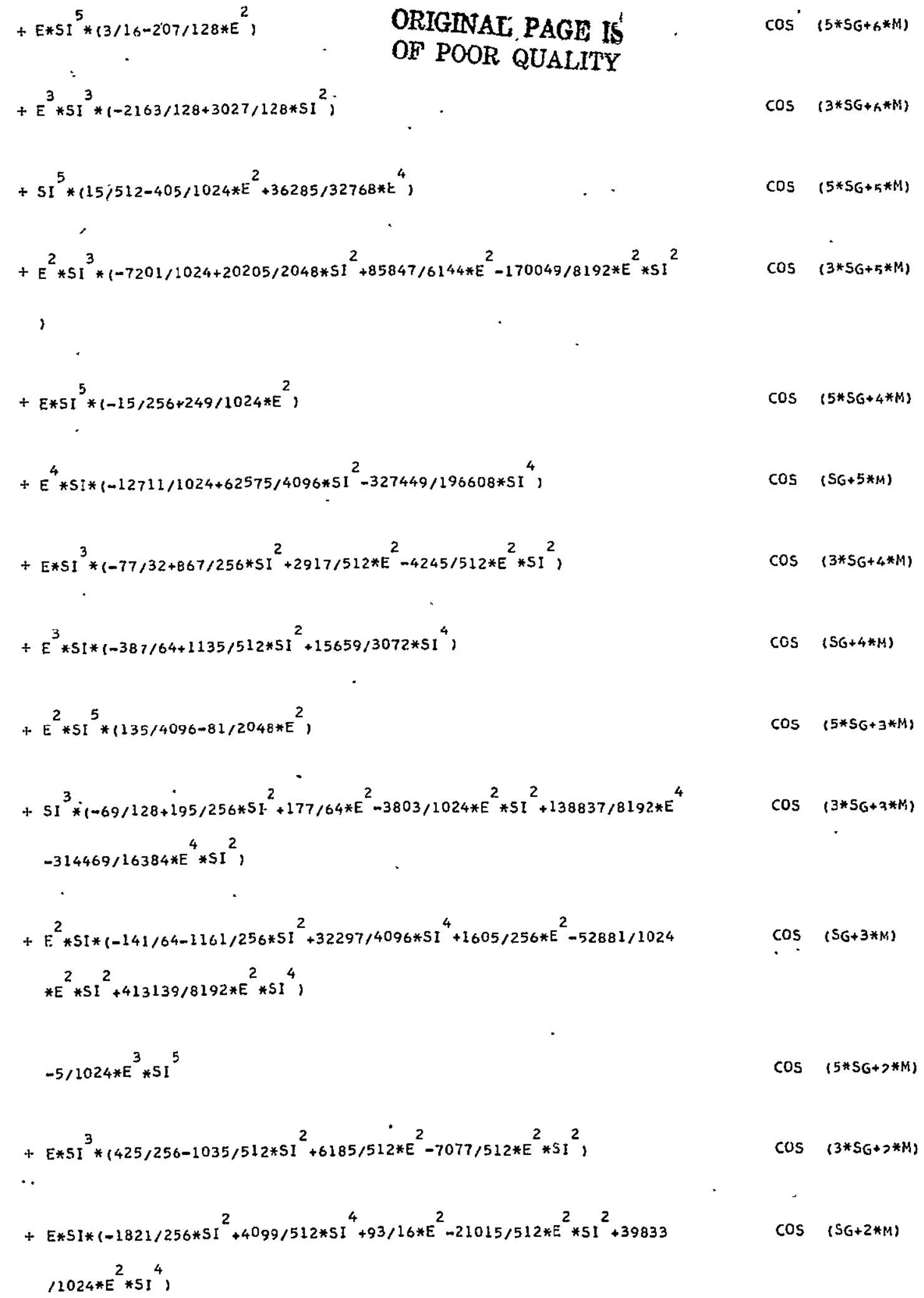




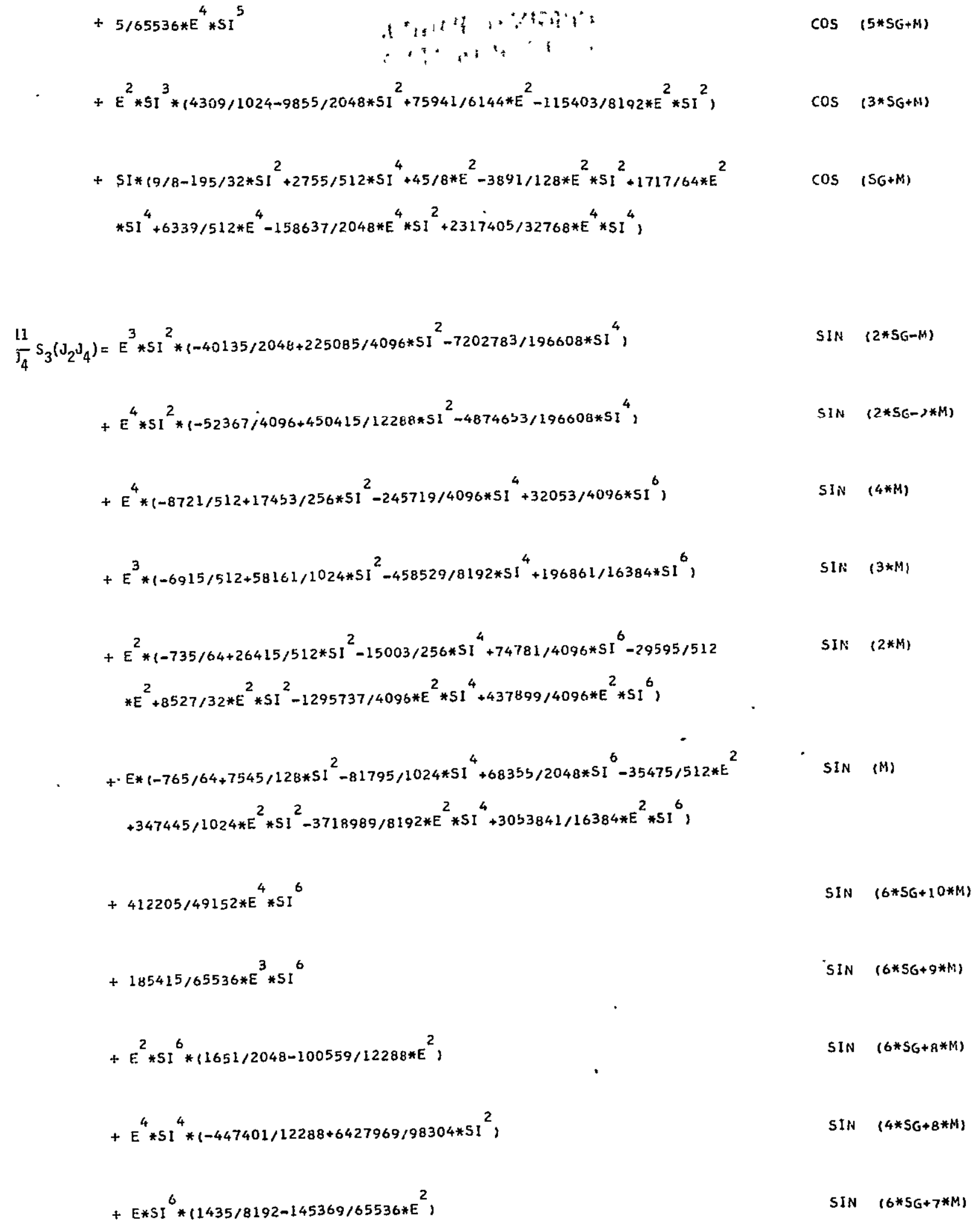


$+E^{3} * \dot{S I}^{4} *\left(-1068423 / 114688+13719047 / 688128 * S 1^{2}\right)$

$+S I^{6} *\left(91 / 4096-3759 / 8192 * E^{2}+135369 / 65536 * E^{4}\right)$

$\operatorname{SIN}(6 * S G+6 * M)$

$+E^{2} * 5 I^{4} *\left(-30,25 / 4096+32371 / 8192 * 5 I^{2}+755605 / 8192 * E^{2}-2105291 / 16384 * E^{2}\right.$

SIN $(4 * 5 G+6 * M)$ $* 5 I^{2}$,

$+E^{4} * 5 I^{2} *\left(-595769 / 4096+1738701 / 4096 * 5 I^{2}-19034635 / 65536 * S I^{4}\right)$

$\operatorname{SIN}(2 * S G+6 * M)$

$+E * S I^{6} *\left(-455 / 8192+25865 / 65536 * E^{2}\right)$

$\operatorname{SIN}(6 * S G+5 * M)$

$+E * 5 I^{4} *\left(7997 / 10240-371 / 4096 * S I^{2}+2358127 / 81920 * E_{0}^{2}-1337549 / 32768 * \varepsilon^{2}\right.$

$\sin (4 * 56+5 * 12)$ $* S I^{2}$,

$+E^{3} * 5 I^{2} *\left(-702799 / 10240+4231921 / 20480 * 5 I^{2}-28358101 / 196608 * 5 I^{4}\right)$

$\operatorname{SIN}(2 * 5 G+5 * M)$

$+E^{2} * S I^{6} *\left(91 / 2048-1505 / 12288 * E^{2}\right)$

$\operatorname{SIN}(6 * S G+\angle * M)$

$+\mathrm{SI}^{4} * 199 / 256-329 / 1024 * S I^{2}+6017 / 1024 * E^{2}-18207 / 2048 * E^{2} * S I^{2}-115609$

$\operatorname{SIN}(4 * 5 G+4 *(N)$ $/ 2048 * E^{4}+283537 / 4096 * E^{4} * S I^{2}$,

$+E^{2} * 5 I^{2} *\left(-3459 / 128+43535 / 512 * 5 I^{2}-31213 / 512 * 5 I^{4}+24863 / 256 * E^{2}-761599\right.$

$\operatorname{SIN}(2 * 5 G+4 * M)$ $13072 * E^{2} * S I^{2}+15145223 / 98304 * E^{2} * 51^{4}$,

$-819 / 65536 * E^{3} * S I^{6}$

SIN $(6 * S G+3 * M)$

$+E * 5 I^{4} *\left(43 / 2048-1589 / 4096 * 5 I^{2}-306991 / 16384 * E^{2}+751821 / 32768 * E^{2} * 5 I^{2}\right)$

$\operatorname{SIN}(4 * 5 G+3 * M)$

$+E * S I^{2} *\left(-1685 / 256+11651 / 512 * S I^{2}-140791 / 8192 * 5 I^{4}+144659 / 2048 * E^{2}\right.$

$\sin (2 * 5 G+3 * M)$ $-802529 / 4096 * E^{2} * S I^{2}+8489153 / 65536 * E^{2} * S I^{4}$, 
$+91 / 98304 * E^{4} * 5 I^{0}$

SIN $(6 * S G+7 * M)$

$+E^{2} * S^{4} *\left(-9197 / 4096+24101 / 8192 * S I^{2}+96331 / 6144 * E^{2}-427189 / 24576 * E^{2}\right.$

$\sin (4 * 5 G+2 * M)$ $* S I^{2}$

$+S^{2} * 175 / 64-1335 / 512 * S I^{2}+5719 / 4096 * \mathrm{SI}^{4}+25275 / 512 * \mathrm{E}^{2}-74621 / 512 * \mathrm{E}$

$\sin (2 * S G+7 * M)$

$* S I^{2}+822549 / 8192 * E^{2} * S I^{4}+178161 / 1024 * E^{4}-4321633 / 8192 * E^{4} * S I^{2}$ $+24202069 / 55536 * E^{4} * \mathrm{SI}^{4}$ )

$+E^{3} * S I^{4} *\left(101313 / 16384-691397 / 98304 * S I^{2}\right)$

$\operatorname{Sin}(4 * 5 G+M)$

$+E * 5 I^{2} *\left(5415 / 256-31385 / 512 * S I^{2}+342587 / 8192 * S I^{4}+207255 / 2048 * E^{2}\right.$

$\operatorname{SIN}(2 * S G+1 H)$ $.-1187761 / 4096 * E^{2} * S I^{2}+12862703 / 65536 * E^{2} * S^{4}$,

\section{A. 2 Expressions of $F^{\star}$}

$F^{*}=F_{1}^{*}+F_{2}^{*}+F_{3}^{*}+F_{4}^{*}$

$F_{1}^{*}=-\frac{J_{2}}{4 L^{3} G^{3}}\left(1-3 c^{2}\right)$

$F_{2}^{*}=F_{2 s}^{*}+F_{2 p}^{*}$

$F_{2 s}^{*}=\frac{3 J_{2}^{2}}{128 L^{3} G^{7}}\left[-5\left(1-2 c^{2}-7 c^{4}\right)+4 \pi\left(1-3 c^{2}\right)^{2}+n^{2}\left(5-18 c^{2}+5 c^{4}\right)\right]-\frac{3 \mathrm{~J}_{4}}{128 L^{3} G^{7}}\left(3-30 c^{2}+35 c^{4}\right)\left(2+3 e^{2}\right)$

$F_{2 p}^{*}=\frac{3 J_{2}^{2}}{64 L^{3} G^{7}} e^{2} s^{2}\left[1-15 c^{2}+\frac{4(1+2 n)}{(1+n)^{2}}\left(1-5 c^{2}\right)\right] \cos 2 g-\frac{3 J_{3}}{8 L^{3} G^{5}} \operatorname{es}\left(1-5 c^{2}\right) \sin g+\frac{15 J_{4}}{64 L^{3} G^{7}} e^{2} s^{2}\left(1-7 c^{2}\right) \cos \cdot 2 g$ 
$A 2 * A 4 * E^{4} / L^{14} * S I^{4} *\left(2685 / 2048-735 / 512 * S I^{2}\right)$

$* A Z^{3} * E^{4} L^{14}{ }^{14} * S I^{4} *\left(1155 / 2048-315 / 512 * 5 I^{2}\right.$,

\section{ORIGINAL PAGE IS}

OF POOR QUALITY

COEFFICIENT OF SIH(3*SG)

$A 2 * A 3 * E^{3} A^{12} * S 1^{3} *\left(-235 / 128+525,256 * \mathrm{SI}^{2}\right)$

COEFFICIENT OF COS $2 *$ SG)

$A 2 * A 4 * E^{2} / L^{14} * 5 I^{2} *\left(755 ; 64-9285 / 256 * 51^{2}+12075 / 512 * 51^{4}+9825 / 128 * E^{2}-107835 / 512 * E^{2} * 51^{2}+70665 / 512 * E^{2} * 5 I^{4}\right)$

$+A Z^{3} * E^{2} L^{14} * 5 I^{2} *\left(5 T^{\prime} 3 / 64-1369 / 64 * 5 I^{2}+3375 / 256 * S I^{4}+11811 / 256 * E^{2}-115221 / 1024 * E^{2} * 5 I^{2}+140865 / 2048 * E^{2} * 5 I^{4}\right)$

COEFFICIENT OF SIN(SG)

$A 2 * A 3 * E / L^{12} * S I * 19 / 4-39 / 16 * S I^{2}+15 / 128 * S I^{4}+9 * E^{2}-693 / 128 * E^{2} * S I^{2}-285 / 64 * E^{2} * S I^{4}$,

COEFFICIENT OF $\operatorname{COS}(0)$,

$A 2 * A 4 / L^{14} *\left(-45 / 16+495 / 32 * S I^{2}-3105 / 128 * 5 I^{4}+3045 / 256 * S_{1}^{6}-675 / 32 * E^{2}+7155 / 64 * E^{2} * 5 I^{2}-5355 / 32 * E^{2} * S I^{4}+20055 / 256 * E^{2} * 51^{6}-5175 / 64 * E^{4}\right.$

$+10732 b / 256 * F^{4} * 51^{2}-311805 / 512 * E^{4} * 51^{4}+565215 / 2048 * \mathrm{~F}^{4} * 51^{6}$,

$+A 2^{3} / L^{14} * 127 / 8-405 / 32 * 51^{2}+2091 / 123 * 5 I^{4}-111 / 16 * 5 I^{6}+621 / 32 * E^{2}-4647 / 64 * E^{2} * S I^{2}+1527 / 16 * E^{2} * S I^{4}-42243,1024 * E^{2} * 5 I^{6}+B 271 / 128 * E^{4}$

$-61959 / 256 * E^{4} * 51^{2}+662529 / 2048 * E^{4} * 5 I^{4}-145701 / 1024 * E^{4} * S 1^{6}$,

$\mathrm{F}_{4}^{\star}$

COEFFICIENT OF SIH(3*SG)

$A 3 * A 4 * E^{3} / L^{16} * S I^{3} *\left\{-5295 / 512+B 955 / 512 * S I^{2}-29925 / 4096 * 5 I^{4}\right.$,

$+A 2^{2} * A 4 * E^{3} / L^{16} * 51^{3} *\left(231 / 8-72645 / 1024 * 51^{2}+176325 / 4096 * S 1^{4}\right)$ 
$A 4^{2} * E^{2} L^{18} * S I^{2} *\left(-14175 / 256+239175 / 1024 * 51^{2}-1320165 / 4096 * 51^{4}+74235 / 512 * 5 I^{6}\right)$

$\div A 3^{2} * E^{2} / L^{14} * S I^{2} *\left(-249 / 32+1965 / 128 * S I^{2}-3975 / 512 * 5 I^{4}\right) ;$

$+A 2^{2} * A 4 * E^{2} L^{18}{ }^{18}{ }^{2}{ }^{2} *\left(-3855 / 128+191775 / 1024 * 51^{2}-2602509 / 8192 * 51^{4}+2626995 / 16364 * 5 I^{6}\right)$

$+A 2^{4} * E^{2} L^{18}{ }^{18} * S I^{2} *\left(11445 / 1024-23853 / 2048 * S I^{2}-157131 / 8192 * 5 I^{4} * 632877 / 32768 * 5 I^{6}\right)$

.

COEFICIENT OF SIN(SG)

$A 3 * A 4 * E / L^{16} * S I *\left(133 / 8-2835 / 32 * S I^{2}+36735 / 236 * S I^{4}-9345 / 128 * S I^{6}+1035 / 8 * E^{2}-345555 / 512 * E^{2} * 51^{2}+559095 / 512 * E^{2} * S I^{4}-1138515 / 2048 * E^{2}\right.$

$* 51^{6}$,

$+A 2^{2} * A 3 * E / L^{16} * S I * 127 / 2-4731 / 64 * S I^{2}+61977 / 512 * S I^{4}-123735 / 2048 * S I^{6} * 6837 / 64 * E^{2}-302487 / 512 * E^{2} * 5 I^{2}+500109 / 512 * E^{2} * 511^{4}-4027665 / 8192$ $* E^{2} * S_{1}^{6}$,

COEFFICIENT OF $\cos (0)$

$A 4^{2} L^{18} *\left(225 / 128-225 / 16 * S I^{2}+20475 / 512 * S I^{4}-94675 / 2048 * 5 I^{6}+152145 / 8192 * 5 I^{8}+675 / 32 * E^{2}-19575 / 128 * E^{2} * S I^{2}+795825 / 2044 * E^{2} * S I^{4}\right.$ $-1660765 / 4096 * E^{2} * 5 I^{6}+153615 / 1024 * E^{2} * S^{8} I^{9}$

$+A 3^{2} \Lambda^{14} *\left(9 / 8-9 * 5 I^{2}+1095 / 64 * S I^{4}-4875 / 512 * 51^{6}+81 / 8 * E^{2}-291 / 4 * E^{2} * S I^{2}+68205 / 512 * E^{2} * S I^{4}-74415 / 1024 * E^{2} * 5 I^{6}\right)$

$+A 2^{2} * A 4 / L^{18} *\left(-135 / 6+7155 / 64 * 5 I^{2}-66525 / 256 * 5 I^{4}+65325 / 256 * 5 I^{6}-23205 / 256 * 5 I^{8}-10935 / 64 * E^{2}+143565 / 128 * E^{2} * 51^{2}-675705 / 256 * E^{2} * 5 I^{4}\right.$

$+10808025 / 4096 * E^{2} * 51^{6}-7816305 / 8192 * E^{2} * 5 t^{B}$,

$+A 2^{4} \lambda^{18} *\left(1755 / 12 \theta-16389 / 256 * 5 I^{2}+24189 / 256 * S I^{4}-94 B 63 / 2048 * S I^{6}+9201 / 8192 * 5 I^{8}+13959 / 128 * E^{2}-16161 / 72 * E^{2} * 5 I^{2}+1506957 / 2048 * E^{2} * S I^{4}\right.$

$-2871795 / 8192 * E^{2} * 51^{6}+1761 / 1024 * E^{2} * 51^{8}$,

A.3 Expressions of $S^{*}$

$s^{*}=s_{1}^{*}+s_{2}^{*}+s_{3}^{*}$

$s_{1}^{*}=-\frac{J_{2}}{32 G^{3}} e^{2} s^{2}\left[\frac{1-15 c^{2}}{1-5 c^{2}}+\frac{4(1+2 n j}{(1+n)^{2}}\right] \sin 2 g-\frac{J_{3}}{2 J_{2}{ }^{6}}$ es $\cos g-\frac{5 J_{4}}{32 J_{2} 6^{3}} \frac{e^{2} s^{2}\left(1-7 c^{2}\right)}{1-5 c^{2}} \sin 2 g$

$s_{2}^{\star}$

COEFFICIENT OF SIM(4*56)

$1 / A 2^{2} * A 4^{2} * E^{4} / L^{7} * S I^{4} *\left(1125 / 512 / M_{G}{ }^{3}-1875 / 256 / I H_{G}{ }^{3} * S I^{2}+16625 / 2048 / M_{G}{ }^{3} * S I^{4}-6125 / 2048 / I N_{G}{ }^{3} * S I^{6}-225 / 256 / 1 H_{G}{ }^{2}+525 / 256 / I N G{ }^{2} * S I^{2}\right.$, $-1225 / 1024 / 1+16^{2} * S^{4} 1^{4}$ 


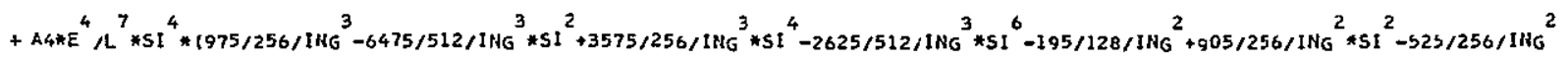
*SI ${ }^{4}-895 / 2048 / I N G+245 / 512 / I N G * S I^{2}$,

$+A Z^{2} * E^{4} / L^{i} * S I^{4} *\left(845 / 512 / 1 N_{G}{ }^{3}-2795 / 512 / 1 N_{G}{ }^{3} * 5 I^{2}+3075 / 512 / 1 N_{G}{ }^{3} * 5 I^{4}-1125 / 512 / 1 N_{G}{ }^{3} * 51^{6}-169 / 256 / 1 N_{G}{ }^{2}+195 / 128 / 1 N_{G}{ }^{2} * 51^{2}-225 / 256 / 1 N_{G}{ }^{2}\right.$ $* 51^{4}-385 / 2048 / I N G+105 / 512 / 1 N G * 5 I^{2}$,

COEFFICIENT OF $\operatorname{COS}(3 *$ SG

ORIGINAL PAGE IS OF POOR QUALITY

$1 / A 2^{2} * A 3 * A 4 * E^{3} / L^{5} * S I^{3} *\left(-15 / 64 / 1 N G{ }^{2}+325 / 128 / I N G^{2} * S I^{2}-175 / 128 / I N G^{2} * S I^{4}+145 / 192 / 111 G-665 / 768 / I H H_{G} * S I^{2}\right)$

$+A 3 * E^{3} \Lambda^{5} * 51^{3} *\left(-65 / 64 / 1 N G^{2} * 35 / 16 / I N G{ }^{2} * S I^{2}-75 / 64 / I M_{G}{ }^{2} * 5 I^{4}-181 / 1152 / I N G+125 / 768 / 11, G * S 1^{2}\right)$

COEFFICIENT OF $\operatorname{SIN}(2 * 5 G)$

$1 / \mathrm{AL}^{2} * A 4^{2} * E^{2} / L^{7} * S I^{2} * 1-75 / 8 / I H G^{2}+3025 / 64 / I N G^{2} * S I^{2}-2275 / 32 / I N G^{2} * S I^{4}+8575 / 256 / 1 N G^{2} * S 1^{6}-2775 / 64 * E^{2}, I N G^{2}+27475 / 12 B * E^{2} / 1 N G^{2} * S I^{2}$

$-163625 / 512 * \Sigma^{2}, 1 \mathrm{NG}^{2} * 81^{4}+153125 / 1024 * E^{2}, 1 \mathrm{NG}^{2} * 51^{6}$,

$+1 / A^{2} 2^{2} * A 3^{2} * \dot{E}^{2} / L^{3} * S I^{2} *\left(-5 / 16 / 1 N G+5 / 16 / 1 N G * S I^{2}+1 / B-15 / 32 * E^{2}, 1 H G+15 / 32 * E^{2} / I N G * S I^{2}+3 / 16 * E^{2}\right.$,

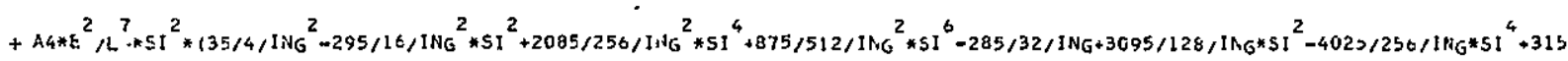
$116 * E^{2} / I N G^{2}-1375 / 128 * E^{2} / I W G^{2} * S I^{2}-26485 / 512 * E^{2} / I N G^{2} * 5 I^{4}+175 / 4 * E^{2} / I N G^{2} * S I^{6}-2135 / 64 * E^{2} / I N G+23565 / 256 * E^{2} / I N G * 5 I^{2}-15505 / 256 * E^{2}$ IIHG*5!

$+A 2^{2} * E^{2} /^{7} * 5 I^{2} *\left(117 / B / I N G^{2}-3277 / 64 / I N G^{2} * 5 I^{2}+15275 / 256 / 1 N G{ }^{2} * 51^{4}-5925 / 256 / 1 N G{ }^{2} * S I^{6}-191 / 32 / I N G+463 / 32 / I N G * S I^{2}-1125 / 128 / I N G * S I^{4}\right.$ $+3283 / 64 * E^{2}, I N G^{2}-11283 / 64 * E^{2}, I N G^{2} * S I^{2}+25765 / 128 * E^{2} / I N G^{2} * S 1^{4}-78175 / 1024 * E^{2} / 1 N G^{2} * S I^{6}-2409 / 128 * E^{2}, I N G+23 b 91 / 512 * E^{2} / 1 N G * S I^{2}$ $-28955 / 1024 * E^{2} /$ ING $^{2}$ SI $^{4}$,

COEFFICIENT OF $\operatorname{COS}(56)$

$1 / A 2^{2} * A 3 * A 4 * E / L^{5} * S I *\left(5, I N G-665 / 32 / 1 N G * S I^{2}+1085 / 64 / 1 N G * S I^{4}+75 / 64 * E^{2} / I N G^{2} * S I^{2}-325 / 128 * E^{2} / I N G^{2} * S I^{4}+175 / 128 * E^{2} / I N G^{2} * S I^{6}+625 / 32\right.$ $* E^{2} / I M G-4975 / 64 * E^{2}, I N G * S I^{2}+15925 / 256 * E^{2} / I R G * S I^{4}$,

*A3*E/L $L^{5} * S I *\left(-6 / I N G+533 / 32 / I N G * S I^{2}-345 / 32 / I H G * S I^{4}+65 / 64 * E^{2} / I N G{ }^{2} * S I^{2}-35 / 16 * E^{2} / I N_{G}{ }^{2} * S I^{4}+75 / 64 * E^{2} / T N^{2} * S I^{6}-469 / 32 * E^{2} / I N G+5131\right.$ $1128 * E^{2}, I H G * S I^{2}-6545 / 256 * E^{2}, I N G * 5 I^{4}$,

$s_{3}^{*}$

COEFFICIENT OF $\operatorname{COS}(3 * S G)$

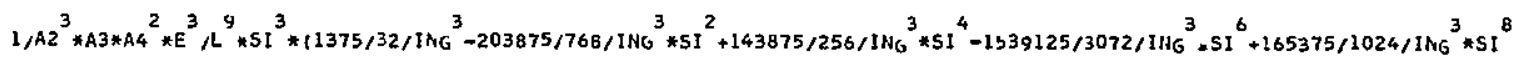
$+10523 / 768 / 1 H G^{2}-48225 / 1024 / 1 \mathrm{NG}^{2} * 5 I^{2}+54775 / 1024 / 1 \mathrm{NG}^{2} * 51^{4}-82075 / 4096 / 1 \mathrm{NG}^{2} * 5 I^{6}$, 


\section{URIGINAL PAGE IS \\ OF POOR QUALITY}

$+1 / A 2^{3} * A 3^{3} * E^{3} / L^{5} * S 1^{3} *\left(25 / 64 / 1 \mathrm{MG}^{2}-25 / 32 / \mathrm{ING}^{2} * 5 \mathrm{I}^{2}+25 / 64 / I \mathrm{NG}^{2} * 5 \mathrm{I}^{4}-305 / 1152 / 1 \mathrm{NG}+305 / 1152 / \mathrm{ING} * 5 \mathrm{I}^{2}+31,576\right)$

$+1 / A 2 * A 3 * A 4 * E^{3} / L^{9} * S I^{3} *\left(-2725 / 96 / I N_{G}{ }^{3}+9775 / 128 / I N_{G}{ }^{3} * S I^{2}-35575 / 768 / 1 N_{G}{ }^{3} * S I^{4}-12225 / 512 / I N_{G}{ }^{3} * S I^{6} * 11375 / 512 / I M_{G}{ }^{3} * S I I^{8}+15755 / 576\right.$ IING ${ }^{2}-1061875 / 9216 / I N G^{2} * S I^{2}+2834725 / 18432 / I N G^{2} * S I^{4}-812875 / 12288 / 1 N_{G}{ }^{2} * S I^{6}-22405 / 1536 / I N_{G}+214505 / 6144 / I H G * S I^{2}-21385 / 1024 / I N_{G}$ $* 5 I^{4}$,

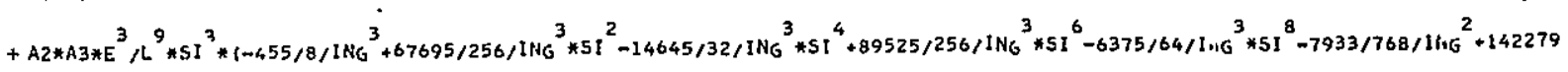

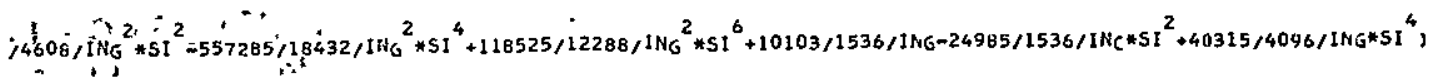
$\therefore ; "$

COEFFICIENT OF SIN(2*SG)

$1 / A 2^{3} * A 4^{3} * E^{2} L^{11} * S^{2} *\left(375 / 4 / 1 N G^{3}-13375 / 16 / 1 N G^{3} * S I^{2}+181375 / 64 / 1 N G^{3} * S I^{4}-291375 / 64 / 1 M^{3} * 5 I^{6}+447125 / 12 B / 1 N G^{3} * 51 * 1586375 / 1536\right.$ $/ I N G^{3} * 5 I^{10}$

$+1 / A 2^{3} * A 3^{2} * A 4 * E^{2} / L^{7} * S 1^{2} *\left(75 / 8 / I N G{ }^{2}-6375 / 128 / I N G{ }^{2} * 5 I^{2}+18925 / 256 / I N G * 5 I^{4}-8575 / 256 / 1 N G{ }^{2} * S I^{6}+115 / 64 / 1 N G-1865 / 768 / 111 G * 5 I^{2}+385\right.$ $11536 / 1$ ING*SI ${ }^{4}$,

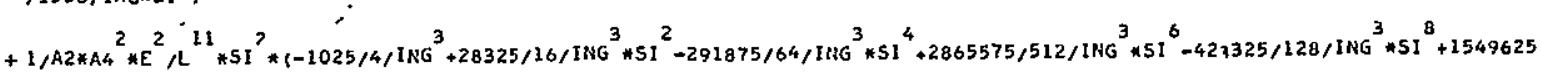

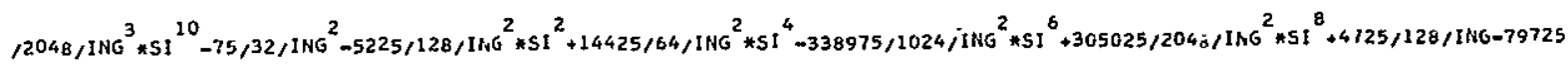
/512/1NG*5I ${ }^{2}+440055 / 2048 / I N G * 5 I^{4}-24745 / 256 / 1 H G * 5 I^{6}$,

$+1 / A 2 * A 3^{2} * E^{2} / L^{7} * S I^{2} * 1-105 / 8 / 1 \mathrm{ING}^{2}+5775 / 128 / 1 N \mathrm{NG}^{2} * S I^{2}-13015 / 256 / \mathrm{ING}^{2} * 51^{4}+4825 / 256 / \mathrm{ING}^{2} * 51^{6}+635 / 192 / I N G-275 / 32 / I N G * 5 I^{2}+355 / 64$ IING*SI ${ }^{4}$,

$+A 2 * A 4 * E^{2} L^{11}{ }^{*} * S I^{2} * 145 / 4 / 1 N G^{3}+60035 / 16 / 1 N G^{3} * S I^{2}-30035 / 16 / 1 N G^{3} * S I^{4}+13235 / 4 / 1 N G^{3} * S I^{6}-10467225 / 4096 / 1 N G^{3} * S I^{8}+6026125 / 8192 / I N G^{3} * S I$

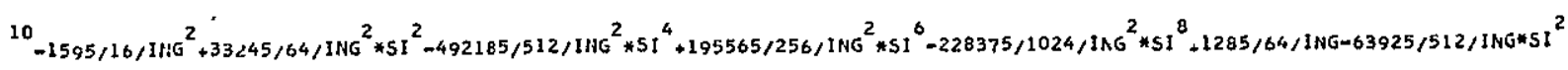
$+867503 / 4096 / 1 N_{G} * 5 I^{4}-875665 / 8192 / 1 N G * S I^{6}$,

$+\mathrm{AL}^{3} * \mathrm{E}^{2} / \mathrm{L}^{11} * 5 \mathrm{I}^{2} * 11053 / 4 / 1 \mathrm{NG}^{3}-24633 / 16 / \mathrm{ING}^{3} * 5 \mathrm{I}^{2}+172861 / 48 / \mathrm{NG}^{3} * \mathrm{SI}^{4}-2156315 / 512 / \mathrm{ING}^{3} * \mathrm{SI}{ }^{6}+10085725 / 4096 / 1 \mathrm{NG}^{3} * \mathrm{SI}^{8}-2358375 / 4096$

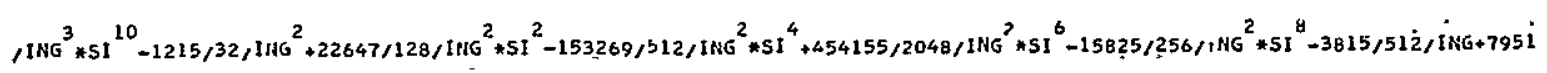

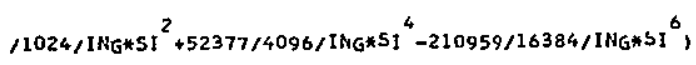

COEFFICIENT OF COS(SG)

$1 / A 2^{3} * A 3 * A 4^{2} * E / L^{2} * 5 I * 1-50 / I N G^{2}+3325 / 8 / I \mathrm{NG}^{2} * S I^{2}-616575 / 512 / I N G^{2} * 5 I^{4}+723275 / 512 / \mathrm{ING}^{2} * 5 I^{6}-3543925 / 6144 / 1 \mathrm{NG}^{2} * \mathrm{SI}^{8}+1125 / 32 * E^{2} / \mathrm{ING}^{3}$

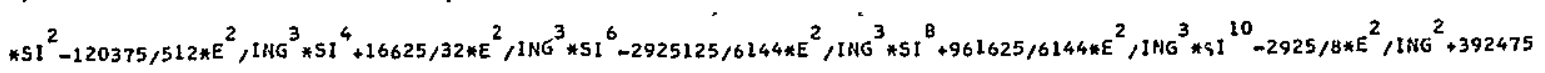
$1128 * E^{2} / I N G^{2} * S I^{2}-71025 / 8 * E^{2} / I N G^{2} * S I^{4}+7975975 / 768 * E^{2} / I N G^{2} * S I^{6}-12986225 / 3072 * E^{2} / I N G^{2} * S I^{8}$,

$+1 / A 2^{3} * A 3^{3} * E / L^{5} * S I *\left(35 / 32 / I N G * 5 I^{2}-35 / 32 / I N G * S I^{4}+1 / 6 * 35 / 48 * S I^{2}-25 / 64 * E^{2} / I N G^{2} * S I^{2}+25 / 32 * E^{2} / I N G^{2} * S I^{4}-25 / 64 * E^{2} / I N G^{2} * S I^{6}-35 / 32 * E^{2}\right.$ $/ I N G+1055 / 128 * E^{2} / 11, G * 5 I^{2}-915 / 128 * E^{2} / I N G * 5 I^{4} * 47 / 48 * E^{2}-575 / 192 * E^{2} * 5 I^{2}$, 
$+1 / A 2 * A 3 * A 4 * E / L^{9} * S I * 1150 / I N G{ }^{2}-15825 / 16 / I N G{ }^{2} * S I^{2}+574175 / 256 / I N G{ }^{2} * 5 I^{4}-1640825 / 768 / 1 N G{ }^{2} * S I^{6}+750575 / 1024 / I N G{ }^{2} * 5 I^{8}+285 / 4 / I N G$ $-21705 / 64 / 1 N G * S I^{2}+125555 / 256 / I N G * S I^{4}-115605 / 512 / I N G * S I^{6}+75 / 32 * E^{2} / I N G^{3} * S I^{2}-13225 / 256 * E^{2} / I N G^{3} * S I^{4}+240175 / 1536 * E^{2} / 1 N G^{3} * S I^{6}$ $-258925 / 1536 * E^{2} / I N G^{3} * S^{8} I^{8}+7875 / 128 * E^{2} / I N G^{3} * 5 I^{10}+13825 / 16 * E^{2} / I N G^{2}-707445 / 128 * E^{2} / I N G^{2} * S I^{2}+12423585 / 1024 * E^{2} / I N G^{2} * S I^{4}-68666125$ $16144 * E^{2} / I_{G}{ }^{2} * S I^{6}+15171975 / 4096 * E^{2} / 1 N_{G}^{2} * S^{8}+25725 / 64 * E^{2} / I N G-934995 / 512 * E^{2} / I N G * S I^{2}+2583775 / 1024 * E^{2} / I N G * S I^{4}-569695 / 512 * E^{2} / I N G$ *SI ${ }^{6}$,

$+A 2 * A 3 * E \cdot L^{9} * S I *\left\{-108 / I N G{ }^{2}+8619 / 16 / I \mathrm{NG}^{2} * 5 I^{2}-1519933 / 1536 / I N G{ }^{2} * S I^{4}+407615 / 512 / I N G{ }^{2} * 5 I^{6}-30375 / 128 / T N G{ }^{2} * S I^{8}-99 / 4 / 1 H G * 4329 / 64 / 1 N G\right.$ $* S I^{2}-7535 / 128 / I N G * S I^{4}+7975 / 512 / I N G * 5 I^{6}-195 / 8 * E^{2} / 1 N G^{3} * S I^{2}+201475 / 1536 * E^{2} / I N G{ }^{3} * S I^{4}-386335 / 1536 * E^{2} / I N G{ }^{3} * S I^{6}+2 U 5975 / 512 * E^{2} / 1 N G{ }^{3}$ $* 5 I^{8}-31875 / 512 * E^{2} / I N G^{3} * S I^{10}-7323 / 16 * E^{2} / I N G^{2}+51769 / 24 * E^{2} / I N G^{2} * 5 I^{2}-11443601 / 3072 * E^{2} / I N G^{2} * 5 I^{4}+b 73 A 015 / 2048 * E^{2} / I N G^{2} * S I I^{0}-3178525$ $14096 * E^{2}, I N G^{2} * 5 I^{8}-6593 / 64 * E^{2}, I N G+129189 / 512 * E^{2}, I N G * 5 I^{2}-366959 / 2048 * E^{2} / I N G * 5 I^{4}+108245 / 4096 * E^{2} / I H G * 5 I^{6}$,

A 4 Expressions of $\mathrm{F}^{\star *}$

$F^{\star *}=F_{1}^{\star \star}+F_{2}^{\star \star}+F_{3}^{\star \star}+F_{4}^{\star \star}$

$F_{1}^{\star \star}=F_{1}^{*}$

$F_{2}^{* *}=F_{2 s}^{*}$

$F_{3}^{\star \star}$ $1 / A 2 * A 4^{2} L^{14} * 1675 / 256 * E^{2} / I N G * S I^{4}-1575 / 256 * E^{2} /$ ING*SI ${ }^{6}+3675 / 1024 * E^{2} /$ ING*SI ${ }^{2}+3375 / 512 * E^{4} / I N G^{2} * S I^{4}-5623 / 256 * E^{4} / I N G^{2} * S I^{6}+49 B 75$ $12048 * E^{4} / I N G^{2} * S I^{8}-18375 / 2048 * E^{4} / I H G^{2} * S I^{10}-675 / 256 * E^{4} / I H G * S I^{2}+15525 / 512 * E^{4} / I N G * S I^{4}-15225 / 256 * E^{4}, I N G * S I^{6}+33075 / 1024 * E^{4}, 1 N G * S I^{8}$

$+1 / A 2 * A 3^{2} L^{10} *\left\{3 / 32 * I N G * S I^{2}-15 / 32 * E^{2} * S I^{2}+15 / 32 * E^{2} * S I^{4}-3 / 32 * E^{2} * I N G+39 / 64 * E^{2} * I N G * S I^{2}-105 / 64 * E^{4} * 5 I^{2}+105 / 64 * E^{4} * S I^{4}-21 / 64 * E^{4} * I N G\right.$ $+441 / 256 * E^{4} * I N G * S^{2}$

$+A 2 * A 4 / L^{14} *\left(-45 / 16+495 / 32 * S I^{2}-3105 / 128 * S I^{4}+3045 / 256 * S I^{6}+585 / 128 * E^{2}, 1 N G * S 1^{4}-2715 / 256 * E^{2}, I N G * 5 I^{6}+1575 / 256 * E^{2}, I N G * 5 I^{6}-675 / 32 * E^{2}\right.$ $+7155 / 64 * E^{2} * S I^{2}-5355 / 32 * E^{2} * S^{4} I^{4}+20055 / 256 * E^{2} * S I^{6}+2925 / 256 * E^{4} / I N G^{2} * S I^{4}-19425 / 512 * E^{4} / I N G^{2} * S I^{6}+10725 / 256 * E^{4} / I N G{ }^{2} * S I^{8} * 7875 / 512 * E^{4}$ ING ${ }^{2} * S I^{10}-585 / 128 * E^{4} / I N G * S I^{2}+27135 / 512 * E^{4} / I N G * 5 I^{4}-106215 / 1024 * E^{4} / I N G * S I^{6}+114975 / 2048 * E^{4} / I N G * S I^{8}-5175 / 64 * E^{4}+107325 / 256 * E^{4}$ $* S I^{2}-311805 / 512 * E^{4} * 5 I^{4}+565215 / 2048 * E^{4} * 5 I^{6}$,

$+A 2^{3} L^{14} *\left(27 / 8-405 / 32 * 5 I^{2}+2091 / 128 * 5 I^{4}-111 / 16 * 5 I^{6}+507 / 256 * E^{2} / I N G * S I^{4}-585 / 128 * E^{2}, I N G * 5 I^{6}+675 / 256 * E^{2} / I N G * 5 I^{8}+621 / 32 * E^{2}-4647 / 64\right.$ $* E^{2} * S I^{2}+1527 / 16 * E^{2} * 5 I^{4}-42243 / 1024 * E^{2} * 5 I^{6}+2535 / 512 * E^{4} / I N G^{2} * S I^{4}-8385 / 512 * E^{4} / I M G^{2} * 5 I^{6}+9225 / 512 * E^{4} / I N G G^{2} * S I I^{8}-3375 / 512 * E^{4} / I N G^{2} * S I$ ${ }^{10}-507 / 256 * E^{4} / I N G * S I^{2}+741 / 32 * E^{4} / I N G * S I^{4}-46305 / 1024 * E^{4} / I N G * S I^{6}+24975 / 1024 * E^{4} / I N G * S I^{8}+8271 / 128 * E^{4}-61959 / 256 * E^{4} * S I^{2}+662529$ $/ 2048 * E^{4} * S^{4}-145707 / 1024 * E^{4} * S I^{6}$, 


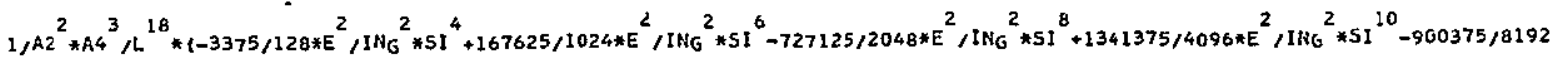
$* E^{2} / I N G^{2} * 5 I^{12}$

$+1 / A 2^{2} * A 3^{2} * A 4 / L^{14} *\left(-15 / 16 * 5 I^{2}+255 / 64 * 5 I^{4}-105 / 32 * 5 I^{6}-225 / 12 B * E^{2} / 1 N G * S I^{4}+975 / 256 * E^{2} / I N G * S I^{6}-525 / 256 * E^{2} / I N G * S 1^{5}+15 / 16 * E^{2}-165 / 8\right.$ $* E^{2} * S I^{2}+1065 / 16 * E^{2} * S 1^{4}-12705 / 256 * E^{2} * S^{6} I^{6}$

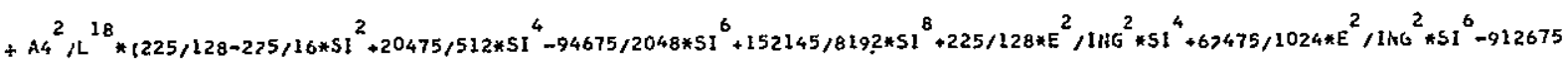
$14096 * E^{2} / I N G^{2} * S^{8}+531825 / 2048 * E^{2} / I N G^{2} * 5 I^{10}-1635375 / 16384 * E^{2} / I N G^{2} * S I^{12}-12825 / 256 * E^{2} / 1 N G * S I^{4}+199125 / 1024 * E^{2} / I^{2}{ }^{2} * 5 I^{6}-126525$ $1512 * E^{2}, I N G * 5 I^{8}+422625 / 4096 * E^{2} ; I N G * S I^{10}+675 / 32 * E^{2}-19575 / 128 * E^{2} * 5 I^{2}+795825 / 2048 * E^{2} * S^{4} I^{4}-1668765 / 4096 * E^{2} * 5 I^{6}+153015 / 1024 * E^{2} * S I^{8}$

,

$+A 3^{2} L^{14} *\left\{9 / 8-135 / 16 * 5 I^{2}+939 / 64 * S I^{4}-975 / 128 * S I^{6}-195 / 128 * E^{2}, I N G * S I^{4}+105 / 32 * E^{2}, I N G * S I^{6}-225 / 128 * E^{2}, I N G * S I^{8}+153 / 16 * E^{2}-9 d^{2} / 16 * E^{2}\right.$

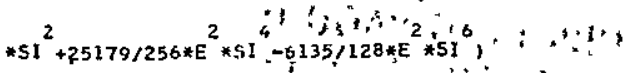

$+A 2^{2} * A 4 / L^{18} *\left(-135 / 8+7155 / 64 * S I^{2}-66525 / 256 * 5 I^{4}+65325 / 256 * 5 I^{6}-23205 / 256 * 5 I^{8}+7995 / 128 * E^{2} / 1 N E^{2} * S I^{4}-767825 / 1024 * E^{2} / 1126^{2} * S I^{6}\right.$

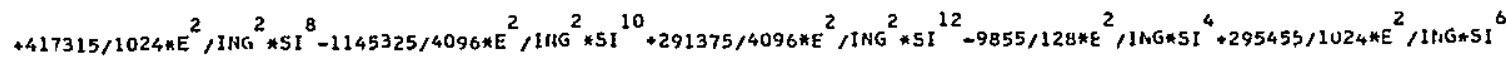
$-731235 / 2048 * E^{2} / I N G * S I^{8}+149625 / 1024 * E^{2} / I N G * S I^{10}-10935 / 64 * E^{2}+143565 / 128 * E^{2} * S I^{2}-675705 / 256 * E^{2} * 5 I^{4}+10808025 / 4096 * E^{2} * 5 I^{6}$ $-7816305 / 8192 * E^{2} * S^{8}$,

$+A 2^{4} \Lambda^{18} *\left(1755 / 128-16389 / 256 * S I^{2}+24189 / 256 * S I^{4}-94863 / 2048 * S I^{6}+9201 / 9192 * 5 I^{8}+4563 / 128 * E^{2} / I N G^{2} * 5 I^{4}-169923 / 1024 * E^{2} / I N 0^{2} * 5 I^{6}\right.$

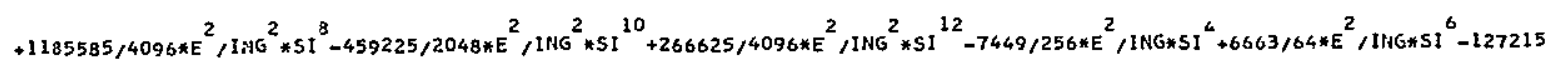
$11024 * E^{2} / I N G * \mathrm{SI}^{8}+50625 / 1024 * E^{2}, I N G * S I^{10}+13959 / 128 * E^{2}-16161 / 32 * E^{2} * \mathrm{SI}^{2}+1506957 / 2048 * E^{2} * S I^{4}-2871795 / 8192 * E^{2} * S I^{6}+1761 / 1024 * E^{2}$ *SI 
APPENDIX B

EXPRESSIONS OF SHORT-PERIOD, LONG-PERIOD, ANO SECULAR PERTURBATIONS

The notation used in the following printout is the same as that in Appendix A; refer to page A-1 for definitions.

B.1 Second-Order Short-Period Perturbations due to $\mathrm{J}_{2}^{2}$

\section{ORIGINAL PAGE IS OF POOR QUALITY}

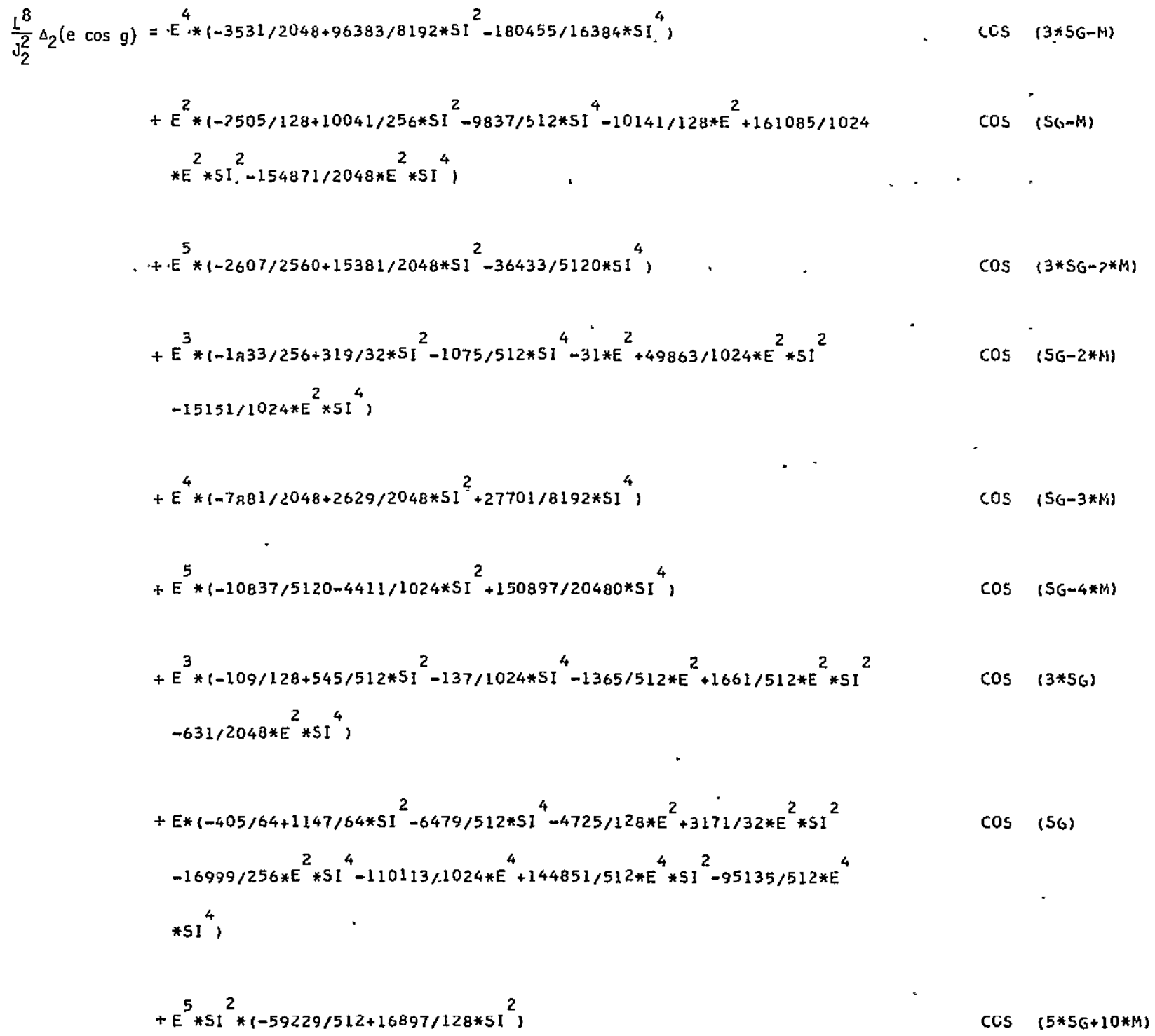




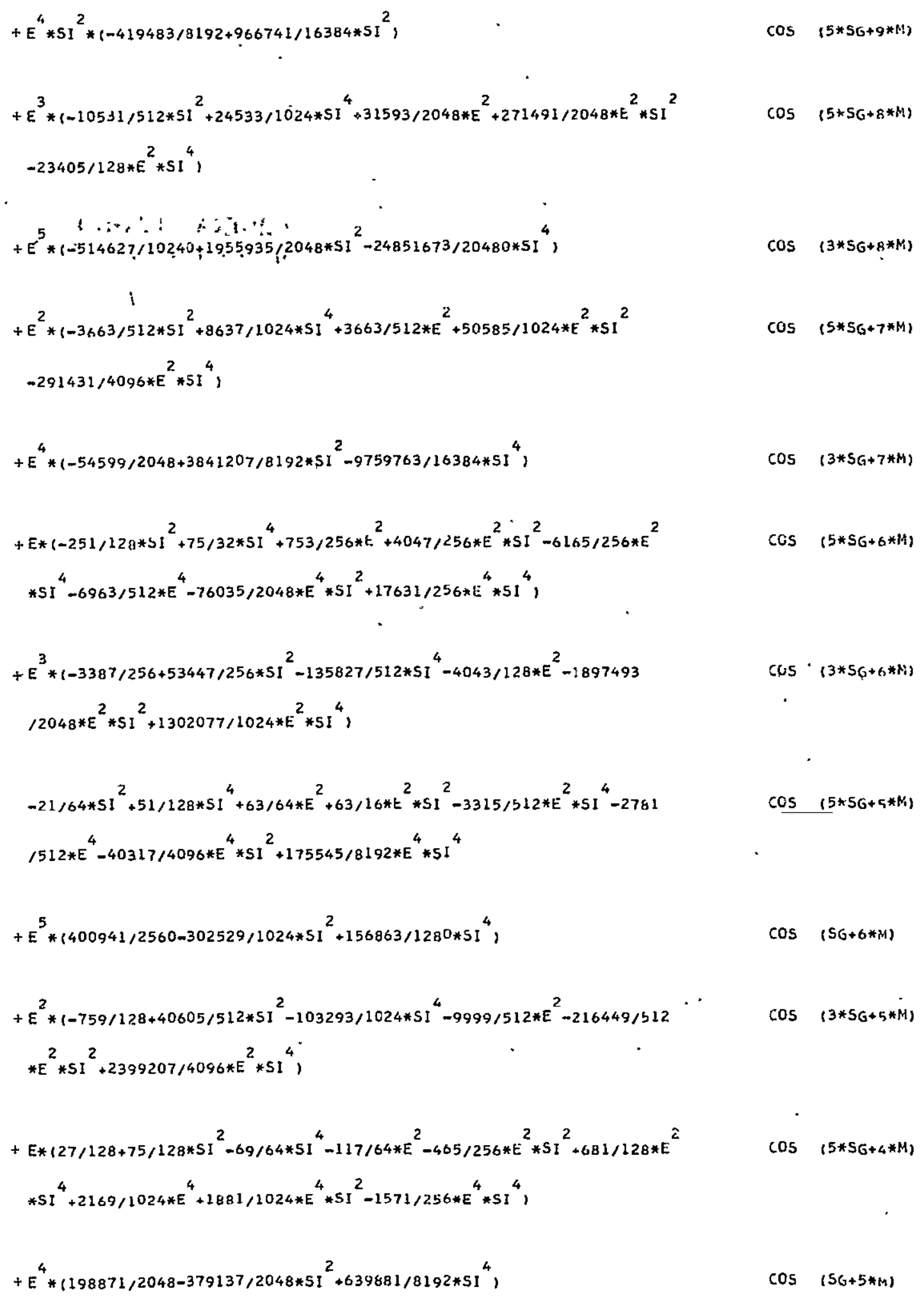




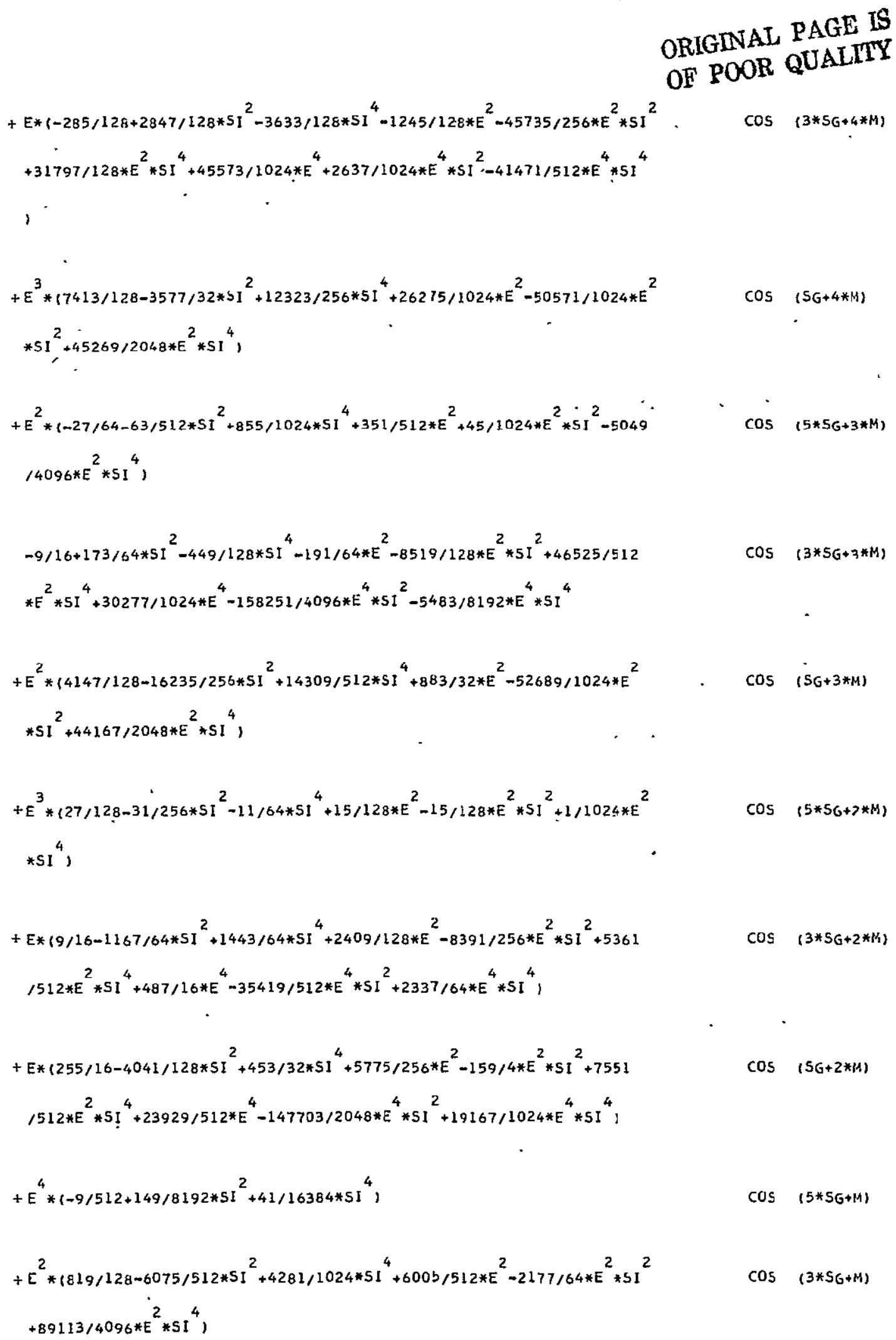


$+81 / 16-75 / 8 * S I^{2}+231 / 64 * S I^{4}+87 / 8 * E^{2}-1269 / 128 * E^{2} * S 1$
$+22953 / 1024 * E^{4}-63 / 512 * E^{4} * 5 I^{2}-133863 / 4096 * E^{4} * 5 I^{4}$

$$
\begin{aligned}
& \frac{L^{8}}{J_{2}^{2}} \Delta_{2}(e \sin g)=\varepsilon^{4} *\left(-3531 / 2048+96377 / 8192 * S I^{2}-180445 / 16384 * 5 I^{4}\right) \\
& +E^{2} * 1-2607 / 128+13485 / 256 * 51^{2}-17163 / 512 * S I^{4}-5401 / 64 * E^{2}+220327 / 1024 \text {. } \\
& * F^{2} * 5 I^{2}-275921,2048 * E^{2} * 5 I^{4} \text {, } \\
& +E^{5} *\left(-2607 / 2560+76881 / 10240 * 5 I^{2}-7285 / 1024 * 51^{4}\right) \\
& +E^{3} * 1-2109 / 256+2.971 / 128 * 5 I^{2}-8023 / 512 * 5 I^{4}-9055 / 256 * E^{2}+24121 / 256 * E^{2} \\
& * S I^{2}-62191 / 1024 * E^{2} * S I^{4} \\
& +\varepsilon^{4} *\left(-10887 / 2048+34583 / 2048 * 51^{2}-100213 / 8192 * 51^{4}\right) \\
& +t^{5} *\left(-21113 / 5120+77861 / 5120 * 51^{2}-48363 / 4096 * 51^{4}\right) . \\
& +E^{3} *\left(-109 / 128+545 / 512 * S I^{2}-137 / 1024 * S I^{4}-1365 / 512 * E^{2}+1661 / 512 * E^{2} * S I^{2}\right. \\
& -631 / 2048 * E^{2} * S I^{4} \text {, } \\
& \begin{array}{l}
+E *\left(-441 / 64+1307 / 64 * S I^{2}-7535 / 512 * 51^{4}-4911 / 128 * E^{2}+26305 / 256 * E^{2} * 5 I^{2}\right. \\
-35075 / 512 * E^{2} * S I^{4}-112777 / 1024 * E^{4}+146279 / 512 * E^{4} * S I^{2}-94841 / 512 * E^{4}
\end{array} \\
& \text { *51 }{ }^{4} \text {, } \\
& +\varepsilon^{5} * 51^{2} *\left(-59229 / 512+16897 / 128 * 51^{2}\right) \\
& +E^{4} * S I^{2} *\left(-419483 / 8192+966741 / 16384 * S I^{2}\right) \\
& +E^{3} * 1-10531 / 512 * S I^{2}+24533 / 1024 * S I^{4}+31593 / 2048 * E^{2}+271491 / 2048 * E^{2} * 5 I^{2} \\
& -23405 / 128 * E^{2} * S^{4} \text {, }
\end{aligned}
$$

$\cos (S G+M)$ 
$+E^{5} *\left(-409317 / 10240+9634329 / 10240 * 5 I^{2}-4869333 / 4096 * 5 I^{4}\right)$

$+E^{2} *\left(-3663 / 512 * S I^{2}+8637 / 1024 * 5 I^{4}+3663 / 512 * E^{2}+50585 / 1024 * E^{2} * S I^{2}\right.$ $-291431 / 4096 * E^{2} * S I^{4}$,

$+E^{4} *\left(-44831 / 2048+3772465 / 8192 * 5 I^{2}-9531233 / 16384 * S I^{4}\right)$

$+E *\left(-251 / 128 * S I^{2}+75 / 32 * 5 I^{4}+753 / 256 * E^{2}+4047 / 256 * E^{2} * 5 I^{2}-6165 / 256 * E^{2}\right.$ $* S I^{4}-6963 / 512 * E^{4}-76035 / 2048 * E^{4} * S I^{2}+17631 / 256 * \varepsilon^{4} * S I^{4}$,

$+E^{3} *\left(-2 B 85 / 256+52257 / 256 * S I^{2}-132003 / 512 * S I^{4}-10407 / 256 * E^{2}-1879953\right.$ $/ 2048 * E^{2} * S I^{2}+1289037 / 1024 * E^{2} * S I^{4}$,

$-21 / 64 * S I^{2}+51 / 128 * S I^{4}+63 / 64 * E^{2}+63 / 16 * E^{2} * S I^{2}-3315 / 512 * E^{2} * 5 I^{4}-2781$ $1512 * E^{4}-40317 / 4096 * E^{4} * S I^{2}+175545 / 8192 * E^{4} * S I^{4}$

$+\varepsilon^{5} *\left(228839 / 2560-356579 / 1280 * S_{I}^{2}+108625 / 512 * S^{4}\right)$

$+E^{2} *\left(-675 / 128+39411 / 512 * 5 I^{2}-99567 / 1024 * 5 I^{4}-11853 / 512 * E^{2}-107119 / 256\right.$ $* E^{2} * S^{2} I^{2}+2374501 / 4096 * E^{2} * S^{4} I^{4}$

$+E * 127 / 128+75 / 128 * 5 I^{2}-69 / 64 * 5 I^{4}-117 / 64 * E^{2}-465 / 256 * E^{2} * S I^{2}+681 / 128 * E^{2}$ $* S^{4}+2169 / 1024 * E^{4}+1881 / 1024 * E^{4} * S I^{2}-1571 / 256 * E^{4} * S I^{4}$,

$+E^{4} *\left(122057 / 2048-374603 / 2048 * S I^{2}+1126583 / 8192 * 5 I^{4}\right)$

$+E *\left(-267 / 128+2721 / 128 * S I^{2}-3441 / 128 * S I^{4}-1401 / 128 * E^{2}-45219 / 256 * E^{2} * S I^{2}\right.$ $+31455 / 128 * E^{2} * S I^{4}+47019 / 1024 * E^{4}+2343 / 1024 * E^{4} * 5 I^{2}-41389 / 512 * E^{4} * 51^{4}$
SIN $(3 * S G+B * M)$

SIN $(5 * 56+7 * M)$

$\sin (3 * 5 G+7 * M)$

$\operatorname{SIN}(5 * \operatorname{Sg}+6 * \%)$

$\operatorname{SIN}(3 * S 6+6 * M)$

$\sin (5 * 56+5 *(M)$

$\sin (S G+6 * M)$

$\operatorname{SIN}(3 * 56+5 * M)$

SIN (5*SG $+4 * M)$

SIN $(56+5 * M)$

$\sin \left(3 * \mathrm{~S}_{6}+4 * M\right)$ 


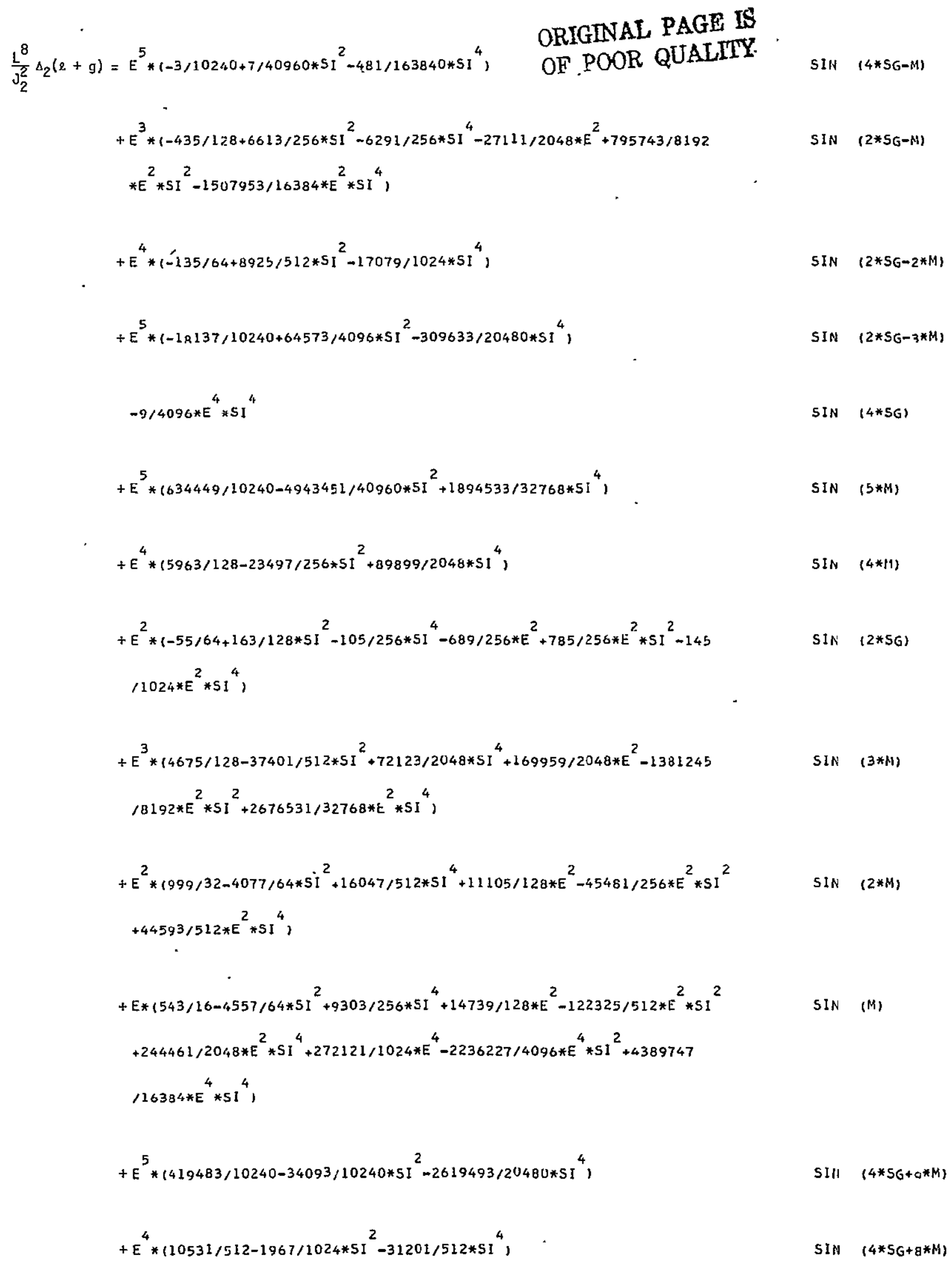




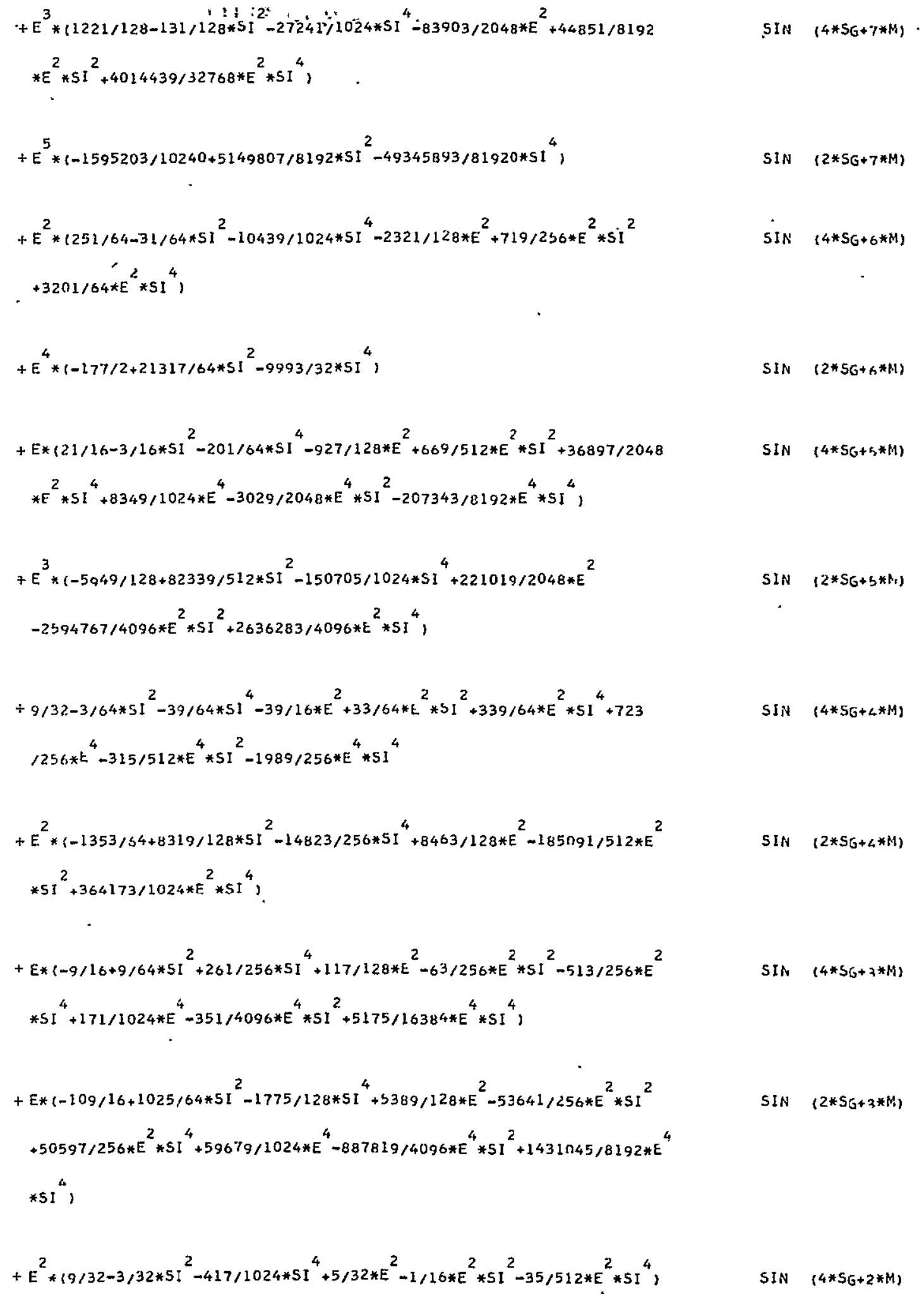


ORIGINAL PAGE IS

OF POOR QUALITI

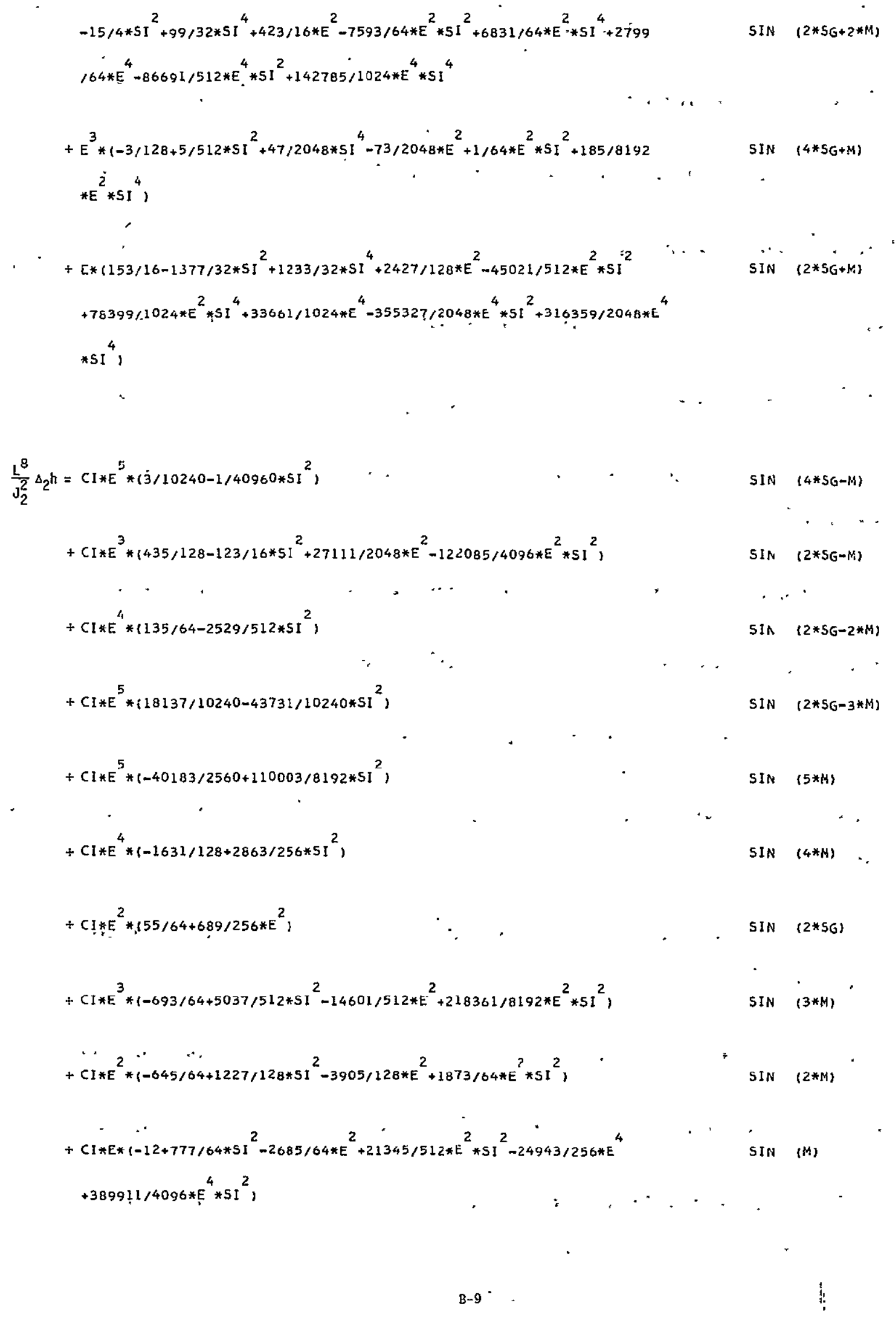


ORIGINAL PAGE IS

OF POOR QUALITY

$+C I * E^{5} *\left(-419483 / 10240-351297 / 20480 * S^{2}{ }^{2}\right)$

$\operatorname{SIN}(4 * S G+Q * M)$

$+C I * E^{4} *\left(-10531 / 512-2141 / 256 * S^{2} I^{2}\right.$

$\operatorname{SIN}(4 * 56+8 * M)$

$+C I * E^{3} *\left(-1221 / 128-959 / 256 * 5 I^{2}+83903 / 2048 * E^{2}+122955 / 8192 * E^{2} * S I^{2}\right) \quad$ SIN $(4 * 5 G+7 * M)$

$* C I * E^{5} *\left(1595203 / 10240-3440013 / 20480 * 51^{2}\right) \quad$ SIN $(2 * 5 G+7 * M)$

$+C I * E^{2} *\left\{-251 / 64-189 / 128 * 5 I^{2}+2321 / 128 * E^{2}+801 / 128 * E^{2} * 5 I^{2}\right) \quad 5 I N \quad(4 * 5 G+6 * M)$

$+C I * E^{4} *\left(177 / 2-48047 / 512 * S^{2}\right) \quad 5 I N \quad\left(2 * S G+6 * N_{i}\right)$

$+C I * E *\left(-21 / 16-15 / 32 * S 1^{2}+927 / 128 * E^{2}+1185 / 512 * E^{2} * S I^{2}-8349 / 1024 * E^{4} \quad\right.$ SIM $(4 * S G+5 * M)$ $-665 / 256 * E^{4} * S^{2}$,

$+C I * E^{3} * 15949 / 128-12279 / 256 * 5 I^{2}-221019 / 2048 * E^{2}+291285 / 2048 * E^{2} * S 1^{2}$,

$\sin (2 * S G+5 * M)$

$+C I *\left(-9 / 32-3 / 32 * S I^{2}+39 / 16 * E^{2}+45 / 64 * E^{2} * S I^{2}-723 / 256 * E^{4}-51 / 64 * E^{4} * S I^{2}, \quad S I N \quad\right.$ (44*SG+4*M)

$+C I * F^{2} *\left(1353 / 64-1323 / 64 * S I^{2}-8463 / 128 * E^{2}+11439 / 128 * E^{2} * 5 I^{2}\right.$,

$\sin (2 * 5 G+4 * M)$

.

$+C I * E *\left(9 / 16+9 / 64 * S I^{2}-117 / 128 * E^{2}-27 / 128 * E^{2} * S I^{2}-171 / 1024 * E^{4}+9 / 4096 * E^{4} \quad\right.$ SIN $(4 * S G+3 * M)$ $* 51^{2}$,

$+C I * E *\left(109 / 16-179 / 32 * 5 I^{2}-5389 / 128 * E^{2}+3707 / 64 * E^{2} * S I^{2}-59679 / 1024 * E^{4} \quad \operatorname{SIN}(2 * S G+3 * M)\right.$ $+172891 / 2048 * E^{4} * 5 I^{2}$,

$+C I * F^{2} *\left(-9 / 32-3 / 64 * 5 I^{2}-5 / 32 * E^{2}-1 / 64 * E^{2} * S I^{2}, \quad 5 \quad S I N \quad(4 * S G+2 * M)\right.$

$+C I * 19 / 8 * S I^{2}-423 / 16 * E^{2}+2349 / 64 * E^{2} * S I^{2}-2799 / 64 * E^{4}+4113 / 64 * E^{4} * 5 I^{2}, \quad$ SIN $(2 * S 6+2 * M)$

$+C I * E^{3} *\left(3 / 128+1 / 512 * S I^{2}+73 / 2048 * E^{2}+9 / 4096 * E^{2} * S I^{2}\right) \quad$ SIN (4*SG+IH) 
ORIGINAL PAGE IS

OF POOR QUALITY

$+C I * E *\left(-153 / 16 * 225 / 16 * S I^{2}-2427 / 128 * E^{2}+8307 / 256 * \dot{E}^{2} * 5 I^{2}-33661 / 1024 * E{ }^{4}\right.$ SIN (2*5G+M) $+65289 / 1024 * E^{4} * 51^{2}$,

$\frac{\mathrm{L}^{7}}{\mathrm{~J}_{2}^{2}} \Delta_{2} \mathrm{~L}=587 / 16384 * \mathrm{E}^{5} * \mathrm{SI}^{4}$

$\cos (4 * 5 G-M)$

$+E^{3} * 5 I^{2} *\left(-121 / 128+15 / 64 * 5 I^{2}-3381 / 4096 * E^{2}-22011 / 8192 * E^{2} * 5 I^{2} 1\right.$

$\cos (2 * S G-M)$

$+E^{4} * 5 I^{2} *\left(-99 / 128-201 / 512 * 5 I^{2}\right)$

$\cos (2 * S G=2 * M)$

$+E^{5} * S I^{2} *\left(-2259 / 5120-28539 / 20480 * 51^{2}\right.$,

$\cos (2 * 5 G=3 * *)$

$+27 / 1024 * E^{4}{ }^{*} \mathrm{SI}^{-4}$

$\cos (4 * S G)$

$+E^{5} *\left(74285 / 1024-11243 / 64 * 5 I^{2}+2341411 / 16384 * 3 I^{4}\right)$

$\cos (5 * 14)$

$+E^{4} *\left(1413 / 32-851 / 8 * 5 I^{2}+21683 / 256 * 5 I^{4}\right)$

$\cos (4 * M)$

$+E^{2} * 5 I^{2} *\left(15 / 32-9 / 8 \pi S I^{2}+225 / 64 * E^{2}-867 / 128 * E^{2} * S I^{2}\right.$,

$\cos (2 * 5 G)$

$+E^{3} *\left(1671 / 6 / 4-4005 / 64 * 5 I^{2}+49779 / 1024 * 5 I^{4}+65349 / 1024 * E^{2}-20475 / 128 * E^{2}\right.$

$\cos (3 * M)$

$* 5 I^{2}+2205063 / 16384 * E^{2} * 5 I^{4}$,

$+E^{2} *\left(237 / 16-1131 / 32 * 5 I^{2}+1707 / 64 * 5 I^{4}+1389 / 32 * E^{2}-431 / 4 * E^{2} * 5 I^{2}+22627\right.$

$\cos (2 * M)$ $1256 * E^{2} * 51^{4}$,

$\div E *\left(63 / 8-75 / 4 * \$ I^{2}+1749 / 128 * S I^{4}+1791 / 64 * E^{2}-4407 / 64 * E^{2} * S I^{2}+56145\right.$

$\cos (M)$ $11024 * E^{2} * S I^{4}+34655 / 512 * E^{4}-21839 / 128 * E^{4} * S I^{2}+1179919 / 8192 * E^{4} * S I^{4}$,

$+809433 / 4096 * E^{5} * S I^{4}$

$\cos (4 * 5 G+q * N)$ 


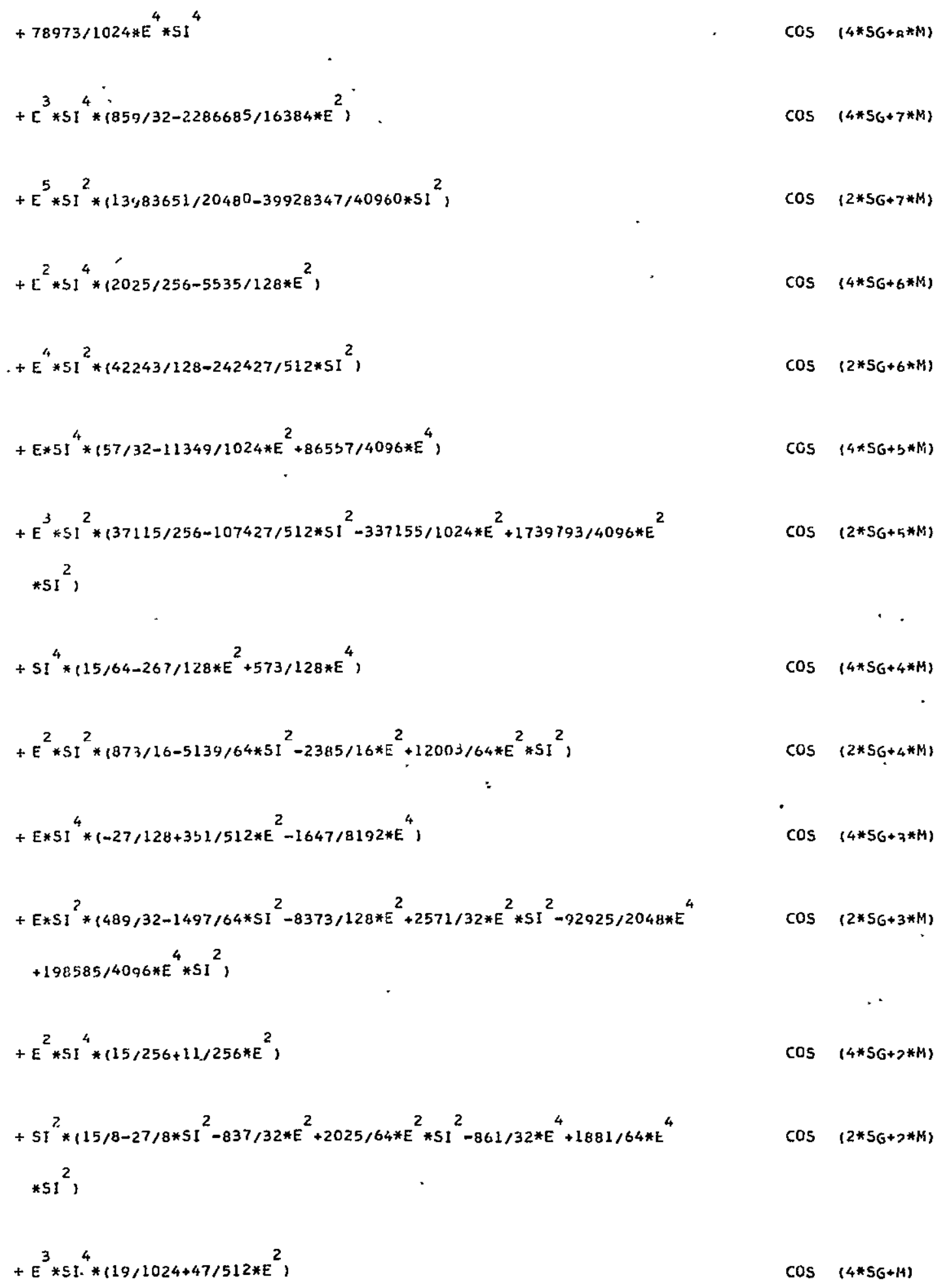




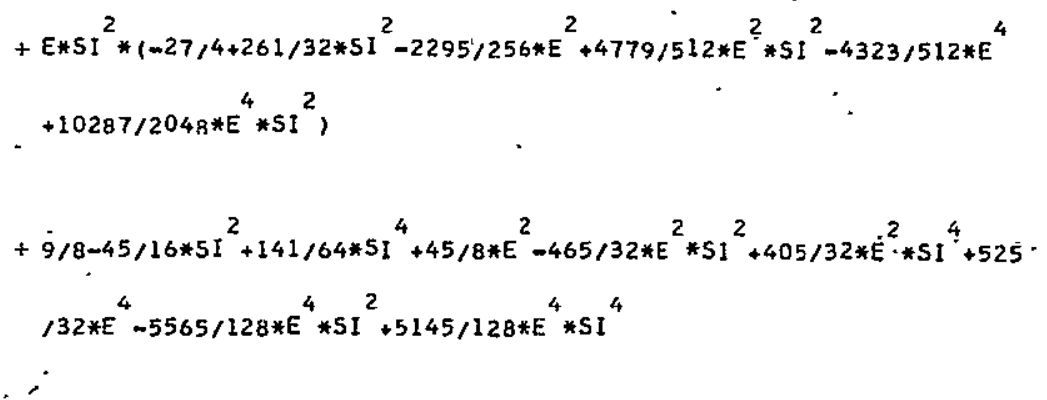

B.2 Third-Order Short-Period Perturbations due to $\mathrm{J}_{2}^{3}$

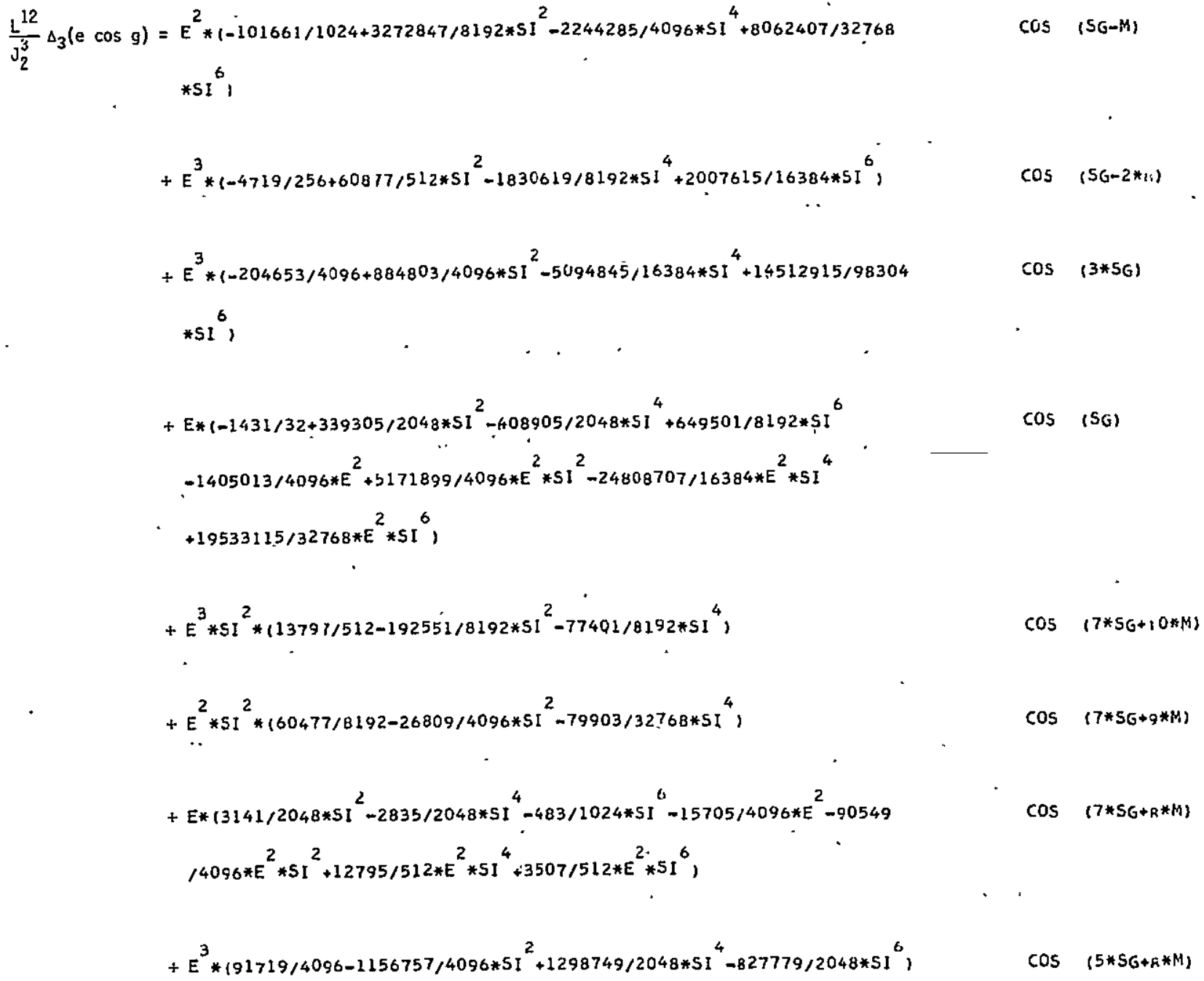




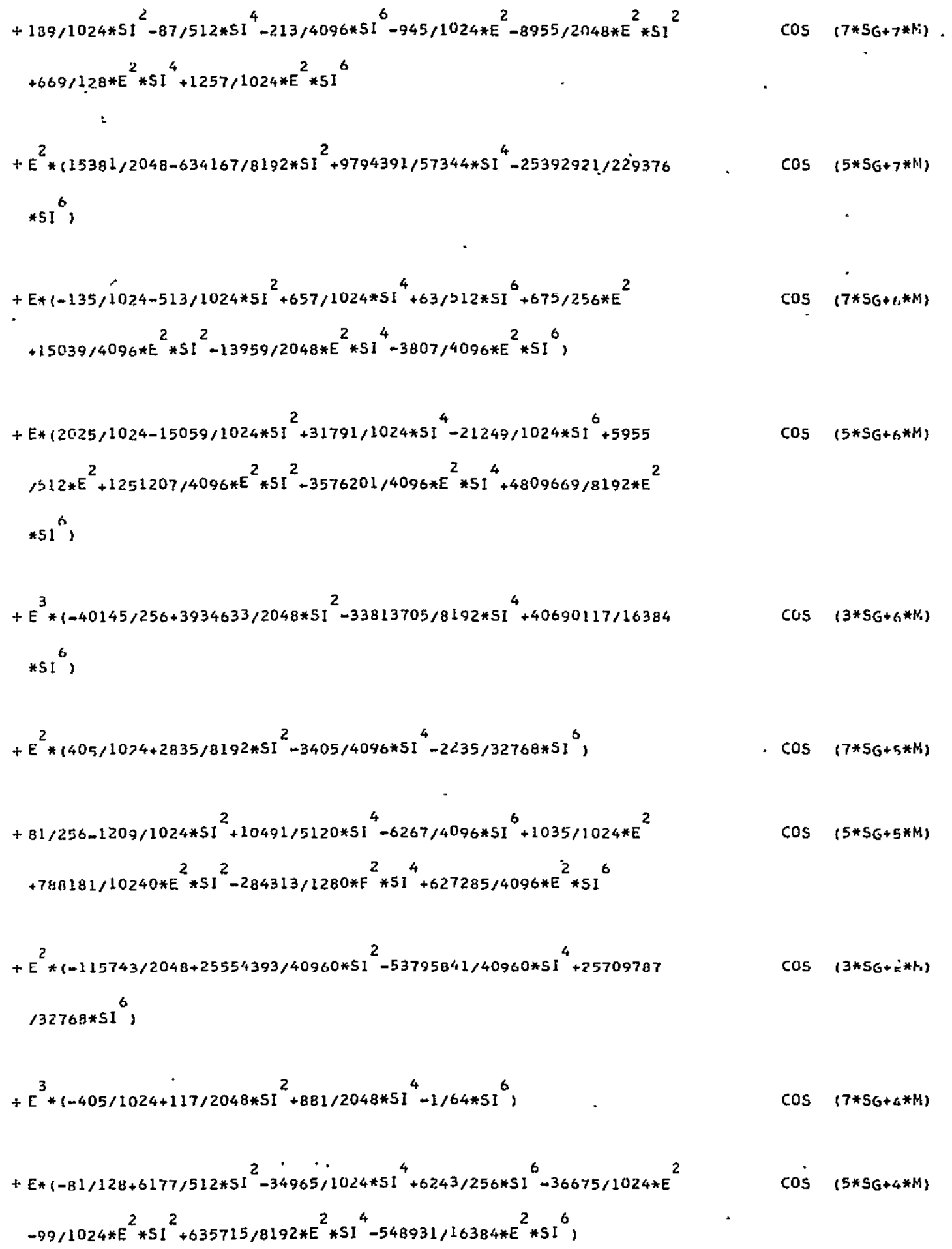




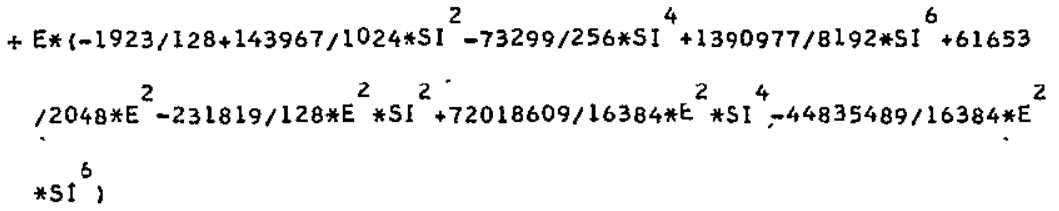

$+2349 / 128-67929 / 1024 * 5 I^{2}+21309 / 256 * 5 I^{4}-137727 / 4096 * 5 I^{6}+40929 / 1024$

$\cos (5 G+M)$ $*^{2}-525045 / 2048 * E^{2} * S I^{2}+494577 / 1024 * E^{2} * S^{4} I^{4}-132759 / 512 * E^{2} * S I^{6}$ 
-

$$
\begin{aligned}
& \frac{L^{12}}{J_{2}^{3}} A_{3}(e \sin g)=E^{2} *\left(-91389 / 1024+2164905 / 8192 * S I^{2}-1035531 / 4096 * S I^{4}+2303577 / 32768 \quad \text { SIN } \quad\right. \text { (SGMM) } \\
& * \mathrm{SI}^{6}, \\
& +E^{3} *\left(-2235 / 256+7003 / 204 B * S I^{2}+239989 / 8192 * S I^{4}-496561 / 16384 * S I^{6}, \quad \text { SIN } \quad\right. \text { (5G-2*M) } \\
& +E^{3} *\left(-204473 / 4096+880537 / 4096 * S I^{2}-15090143 / 49152 * S I^{4}+1419 B 011 \quad\right. \text { SIN (3*SG) } \\
& 198304 * 51^{6} \text {, } \\
& +E *\left(-1215 / 32+250687 / 2048 * 5 I^{2}-250215 / 2048 * 5 I^{4}+308811 / 8192 * S I^{6} \quad\right. \text { SIN (SG) } \\
& -1299347 / 4096 * E^{2}+3986665 / 4096 * E^{2} * S 1^{2}-15143357 / 16384 * E^{2} * 51^{4} \\
& +8638517 / 32768 * E^{2} * 5 I^{6} \text {, } \\
& +E^{3} * 51^{2} *\left(13797 / 512-192551 / 8192 * 51^{2}-77401 / 8192 * 51^{4}\right) \\
& +E^{2} * S I^{2} *\left(60477 / 8192-26809 / 4096 * 5 I^{2}-79903 / 32768 * S I^{4}\right) \quad \text { SIN }(7 * 5 G+9 * M) \\
& +E *\left(3141 / 2048 * S I^{2}-2835 / 2048 * S I^{4}-483 / 1024 * 5 I^{6}-15705 / 4096 * E^{2}-90549 \quad \text { SIN }(7 * 5 G+8 * M)\right. \\
& 14096 * E^{2} * S^{2}+12795 / 512 * E^{2} * S^{4}+3507 / 512 * E^{2} * S^{6}, \\
& +E^{3} *\left(85437 / 4096-1143413 / 4096 * S I^{2}+82369 / 128 * S I^{4}-857985 / 2048 * S I^{6}\right) \quad \text { SIN }(5 * S G+8 * N) \\
& +189 / 1024 * 51^{2}-87 / 512 * S I^{4}-213 / 4096 * 5 I^{6}-945 / 1024 * E^{2}-8955 / 2048 * E^{2} * 5 I^{2} \quad \text { SIN }(7 * S G+7 * M) \\
& +669 / 128 * E^{2} * 5 I^{4}+1257 / 1024 * E^{2} * 5 I^{6} \\
& +E^{2} *\left(14625 / 2048-624765 / 8192 * 5 I^{2}+9978099 / 57344 * S I^{4}-26563587 / 229376 \quad \text { SIN } \quad(5 * 5 G+7 * M)\right. \\
& \text { *SI }{ }^{6} \text {, } \\
& +E *\left(-135 / 1024-513 / 1024 * S I^{2}+657 / 1024 * S I^{4}+63 / 512 * S I^{6}+675 / 256 * E^{2} \quad \text { SIN }\left(7 * S G+6 * N_{1}\right)\right. \\
& +15039 / 4096 * E^{2} * S I^{2}-13959 / 2048 * E^{2} * S I^{4}-3807 / 4096 * E^{2} * S I^{6} \text {, }
\end{aligned}
$$




\section{ORIGINAL PAQR IS \\ OF POOR QUAJIT}

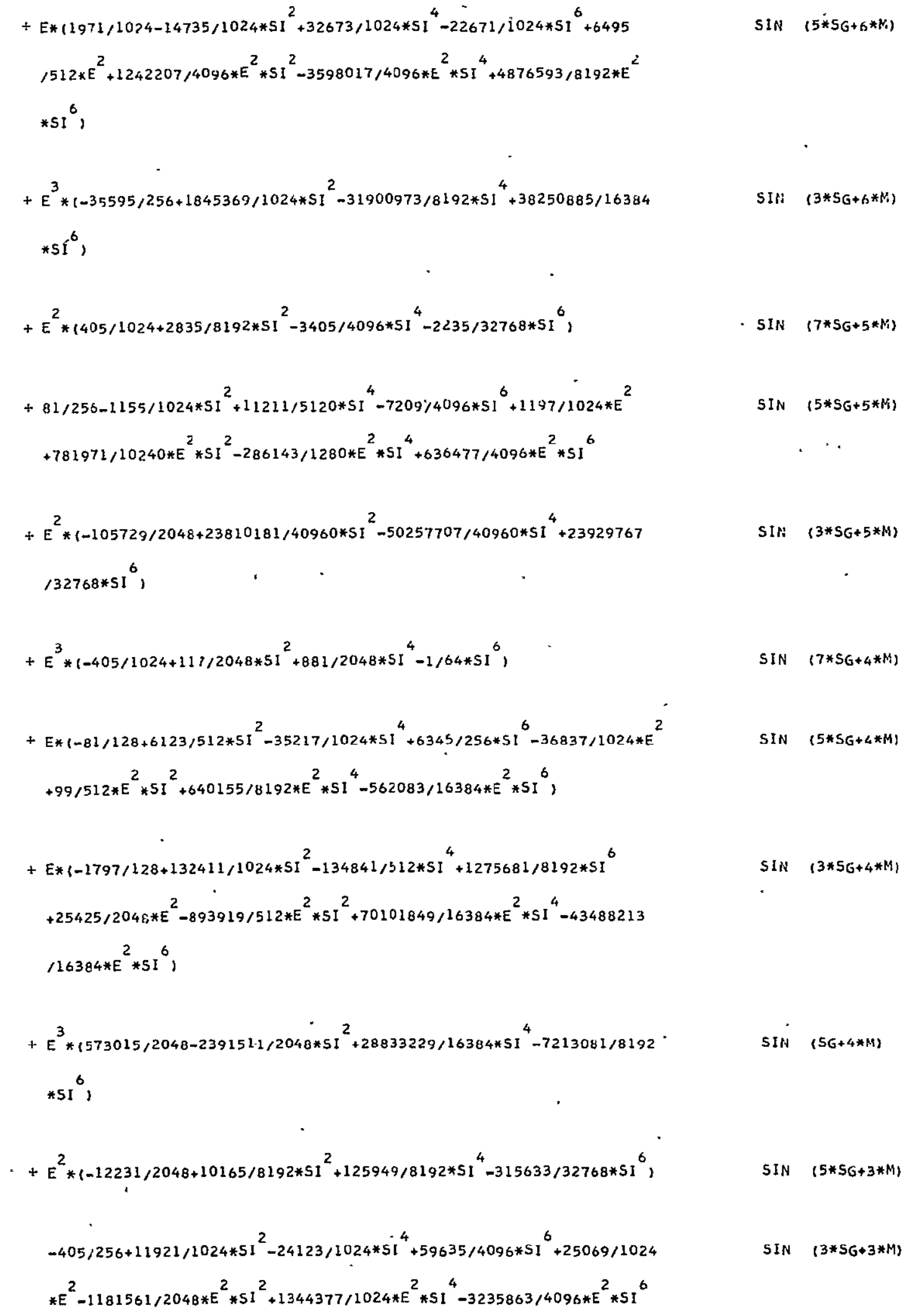




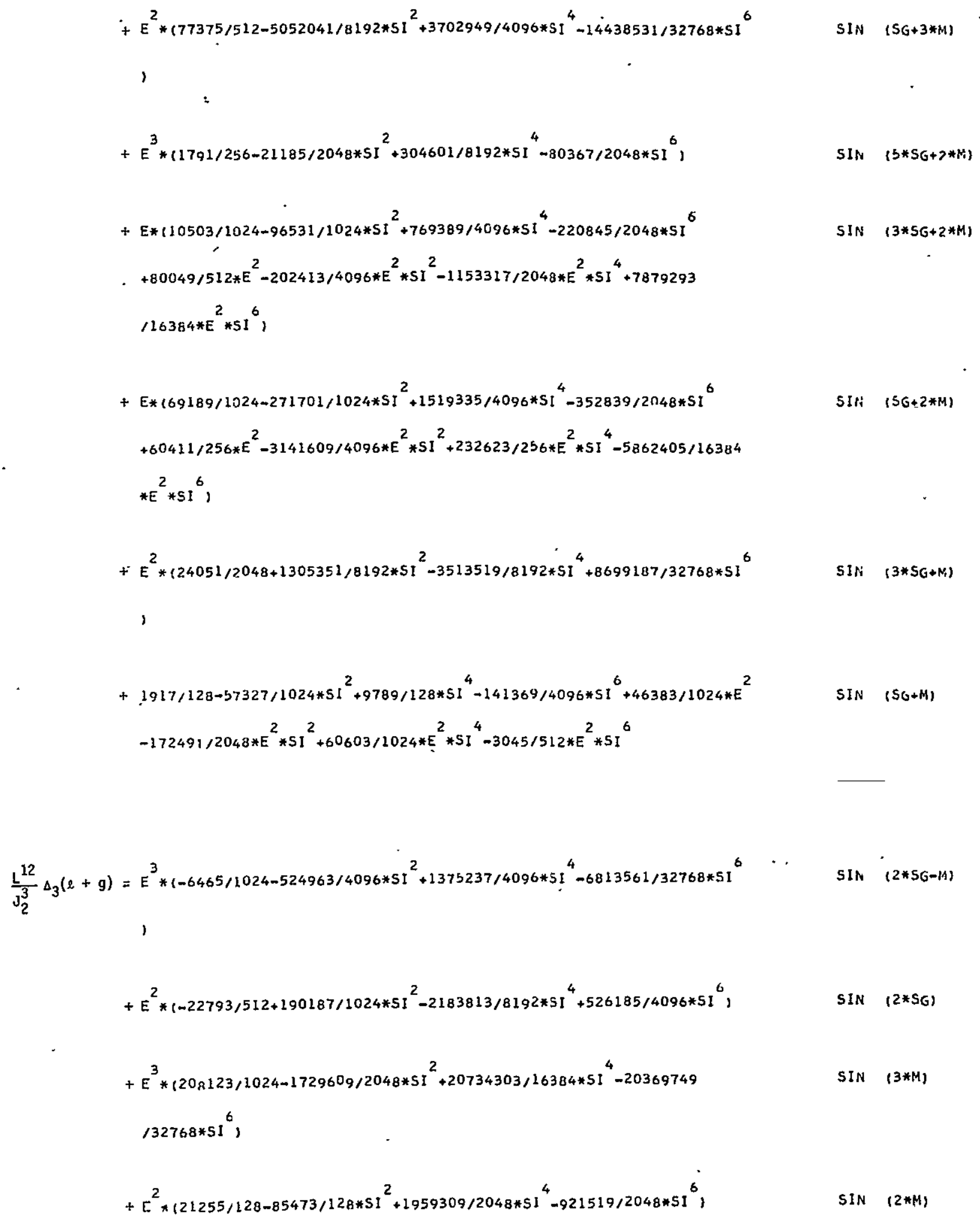




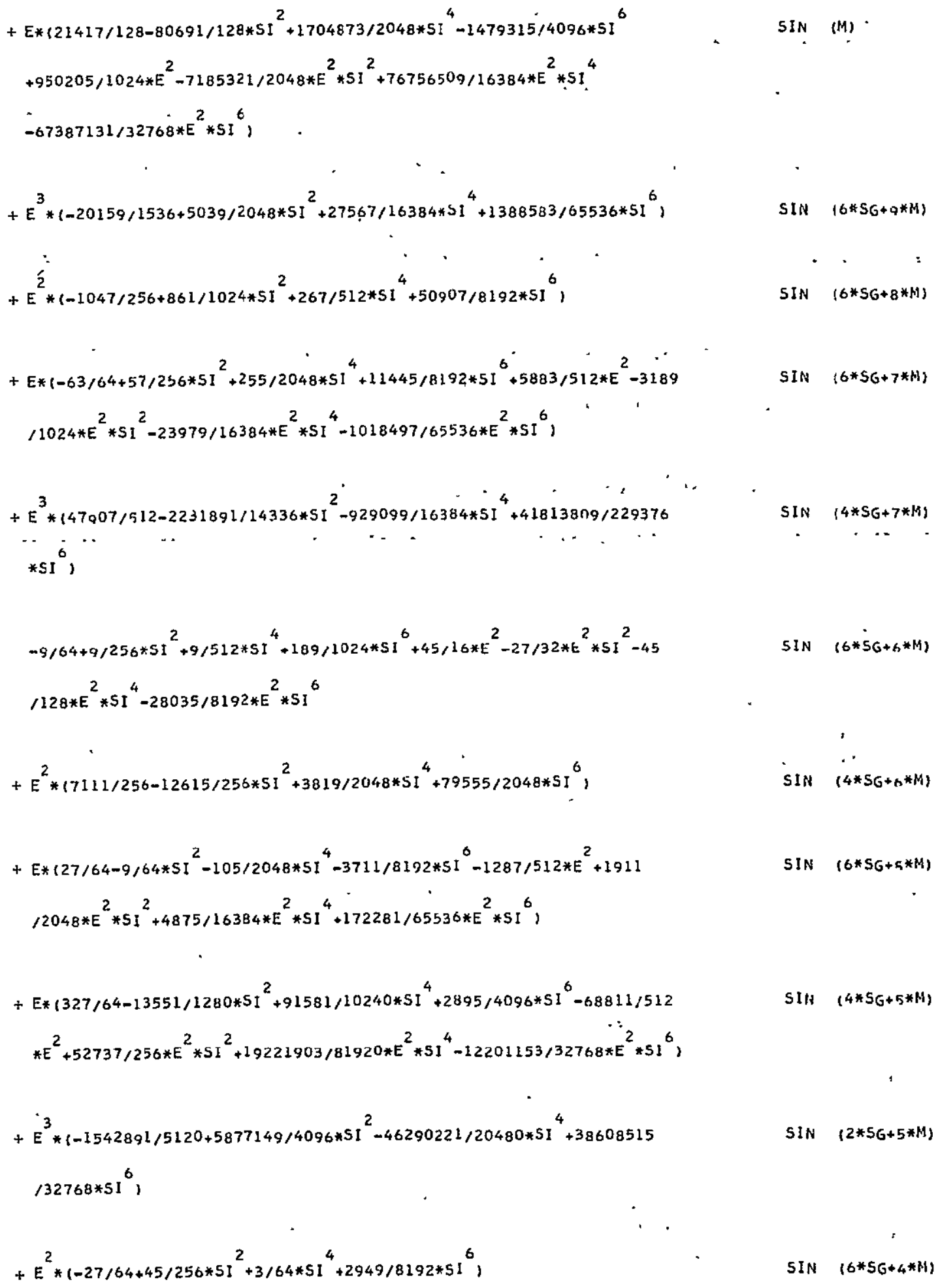




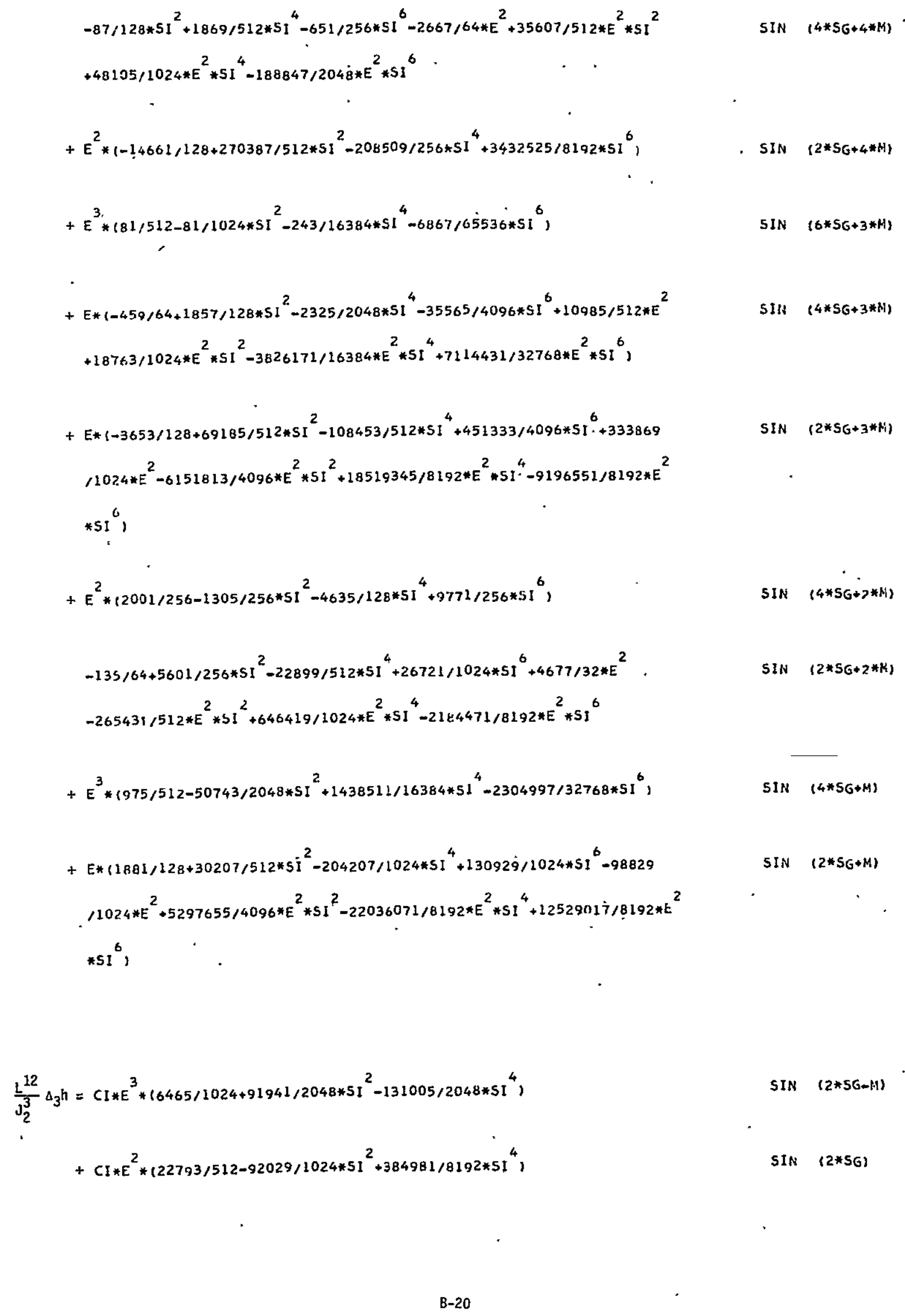


$+C I * E^{3} *\left(-36641 / 512+250197 / 1024 * S I^{2}-1548013 / 8192 * 5 I^{4}\right.$

$\sin (3 * M)$

$+\dot{C}_{\left.I * E^{2} * 1-7849 / 128+50595 / 256 * 5 I^{2}-300093 / 2048 * S I^{4}\right)}$

$\sin (2 * M)$

$+C I * E *\left(-4107 / 64+46197 / 256 * S I^{2}-125169 / 1024 * S I^{4}-186237 / 512 * E^{2}\right.$

SIN (M) $+1054935 / 1024 * E^{2} * S I^{2}-5798205 / 8192 * t^{2} * S I^{4}$,

$+C I * E^{3} *\left(20159 / 1536+25201 / 6144 * S I^{2}+32913 / 16384 * 5 I^{4}\right)$

$\sin (6 * S G+9 * M)$

$+C I * E^{2} *\left(1047 / 256+1233 / 1024 * 5 I^{2}+303 / 512 * S I^{4}\right)$

SIN $(6 * S G+8 * M)$

$+C I * E *\left(63 / 64+69 / 256 * S I^{2}+273 / 2048 * 5 I^{4}-5883 / 512 * E^{2}-1347 / 512 * E^{2} * 5 I^{2}\right.$

SIN $(6 * 5 G+7 * M)$ $-21105 / 16384 * E^{2} * 51^{4}$,

$+C I * E^{3} *\left(-47907 / 512+1501193 / 14336 * S I^{2}+361763 / 57344 * S I^{4}\right)$

$\operatorname{SIN}(4 * S G+7 * M)$

$+C I *\left(9 / 64+9 / 256 * S I^{2}+9 / 512 * 5 I^{4}-45 / 16 * E^{2}-9 / 16 * E^{2} * 5 I^{2}-9 / 32 * E^{2} * S I^{4}\right.$,

SIN $(6 * S G+6 * N)$

$+C I * E^{2} *\left(-7111 / 256+18119 / 512 * S I^{2}-4737 / 2048 * S 1^{4}\right)$

$\operatorname{SIN}(4 * S G+6 * M)$

$+C I * E *\left(-27 / 64-9 / 128 * S I^{2}-75 / 2048 * 5 I^{4}+1287 / 512 * E^{2}+663 / 2048 * E^{2} * S I^{2}\right.$

$\operatorname{SIN}(6 * S G+5 * M)$ $+2925 / 16384 * E^{2} * S I^{4}$,

$+C I * E *\left(-327 / 64+10281 / 1280 * S I^{2}-2577 / 1024 * S I^{4}+68811 / 512 * E^{2}-142137\right.$

$5 I N(4 * S G+5 * M)$ $11024 * E^{2} * S 1^{2}-366837 / 8192 * E^{2} * S I^{4}$,

$+C I * E^{3} *\left(1542891 / 5120-3481719 / 5120 * 5 I^{2}+774991 / 2048 * 51^{4}\right)$

$\sin (2 * S G+5 * M)$

$+C I * E^{2} *\left(27 / 64+9 / 256 * S I^{2}+3 / 128 * S I^{4}\right)$

SIN $(6 * 5 G+4 * *)$

$\div C I *\left(87 / 128 * S I^{2}-15 / 16 * S I^{4}+2667 / 64 * E^{2}-24939 / 512 * E^{2} * S I^{2}-1005 / 128 * E^{2}\right.$

$\operatorname{SIN}(4 * S G+4 * N)$ *SI ${ }^{4}$, 


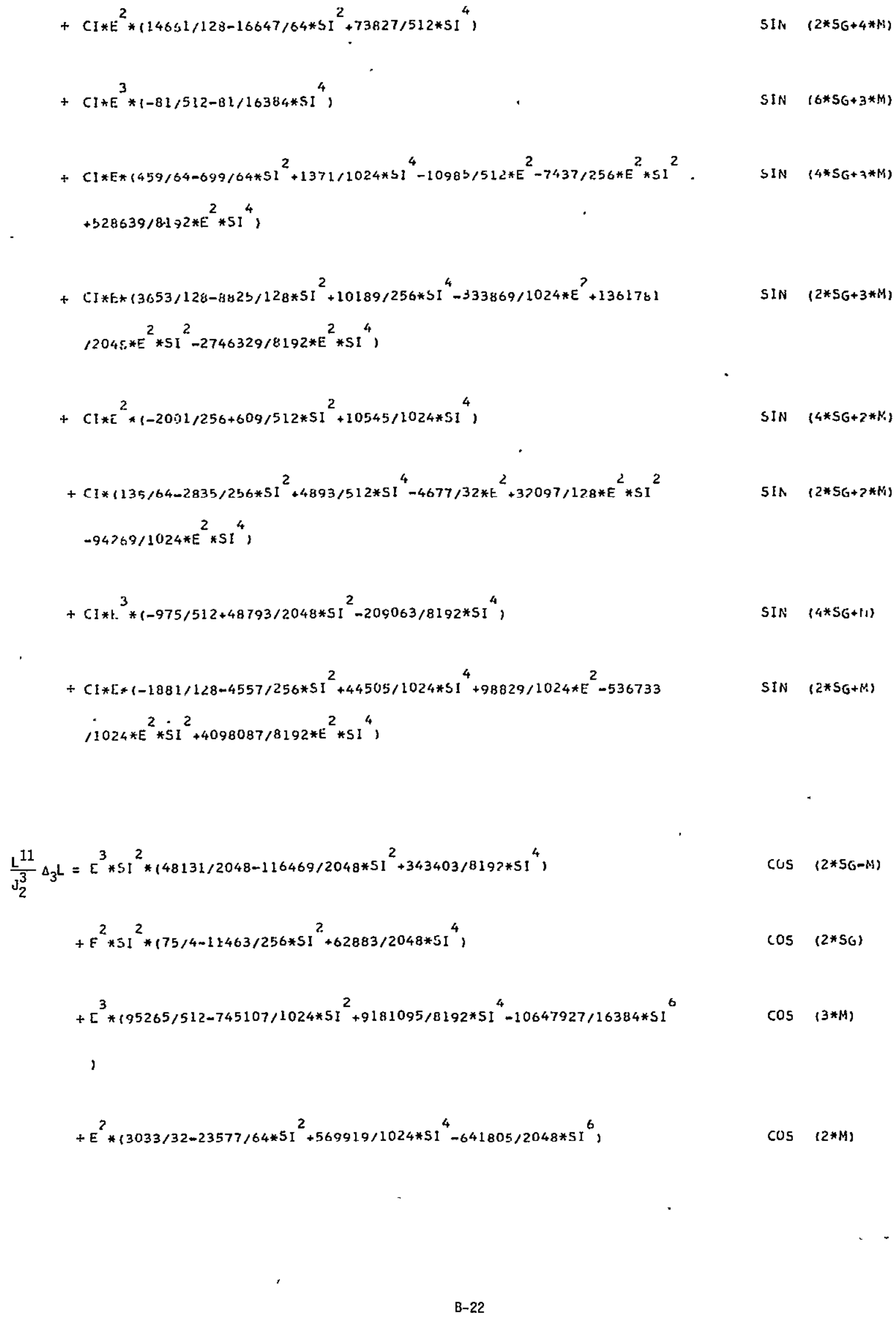




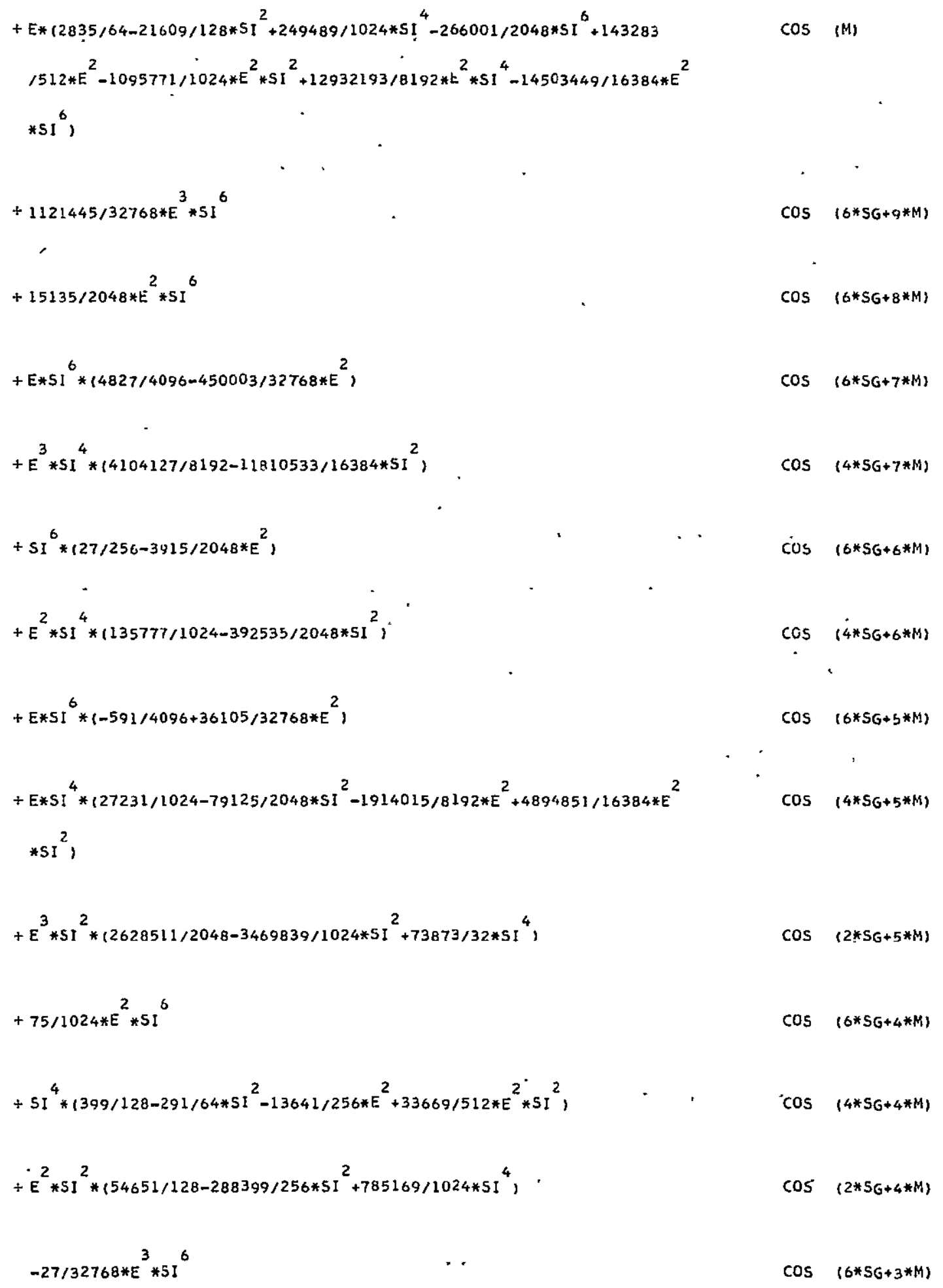




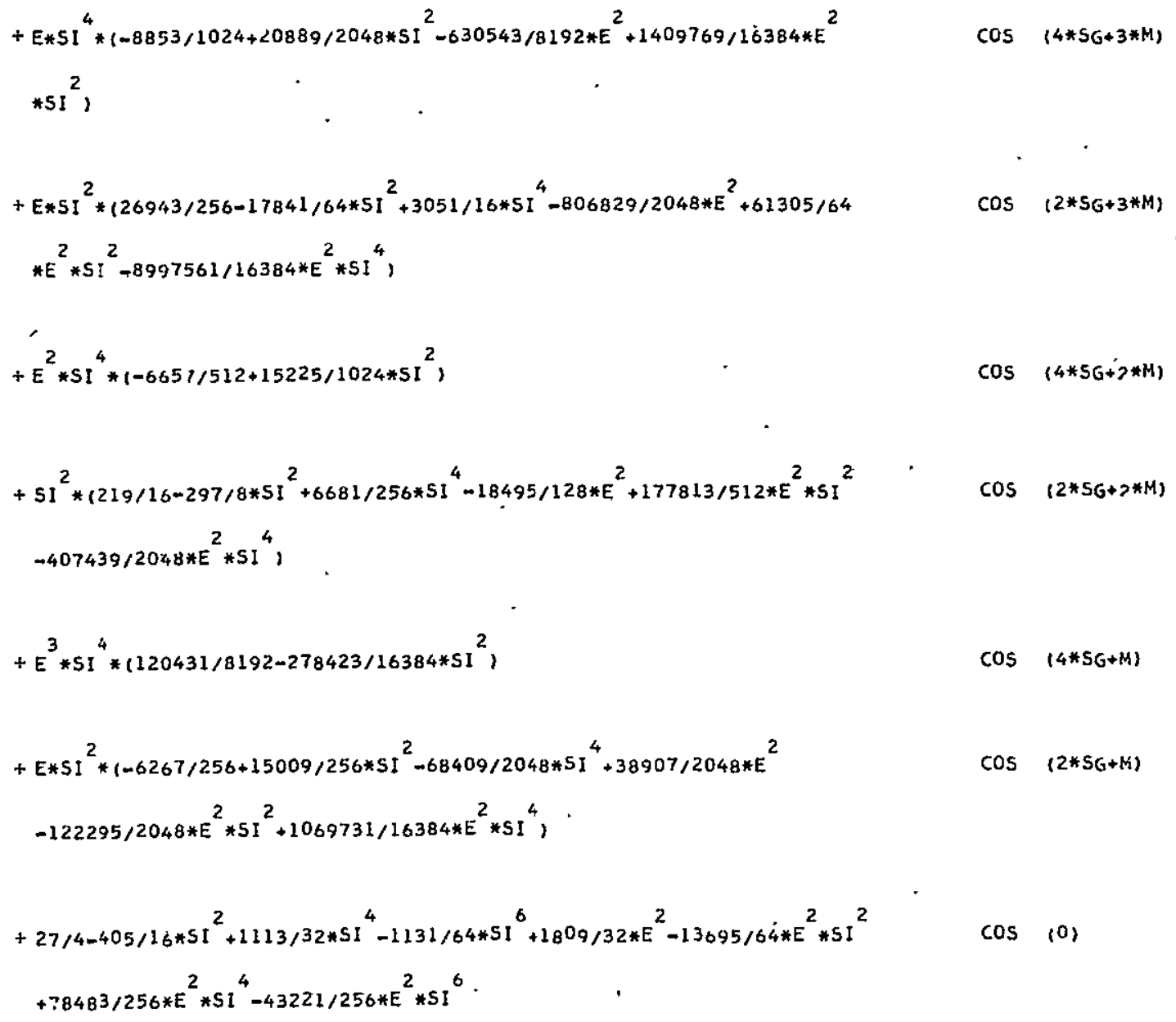

B. 3 Third-Order Short-Period Perturbations due to $\mathrm{J}_{2} \mathrm{~J}_{3}$

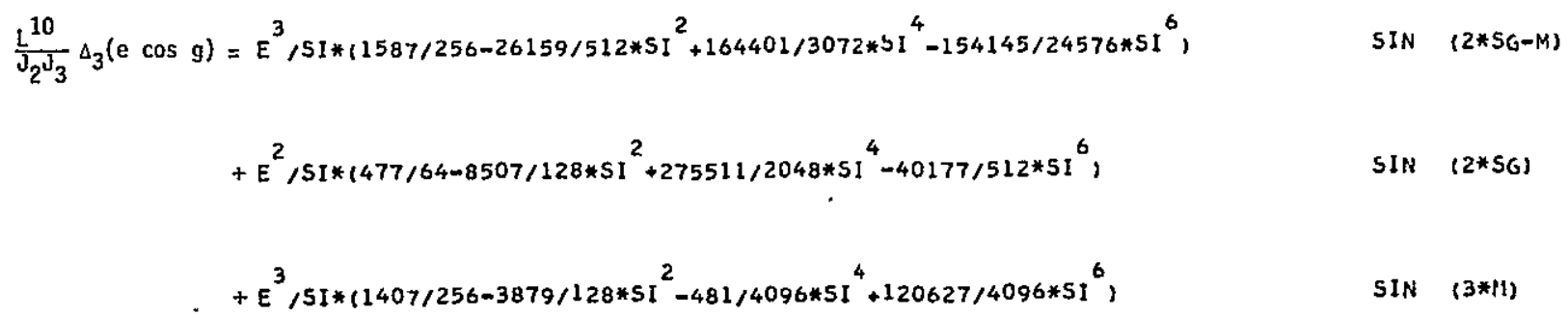




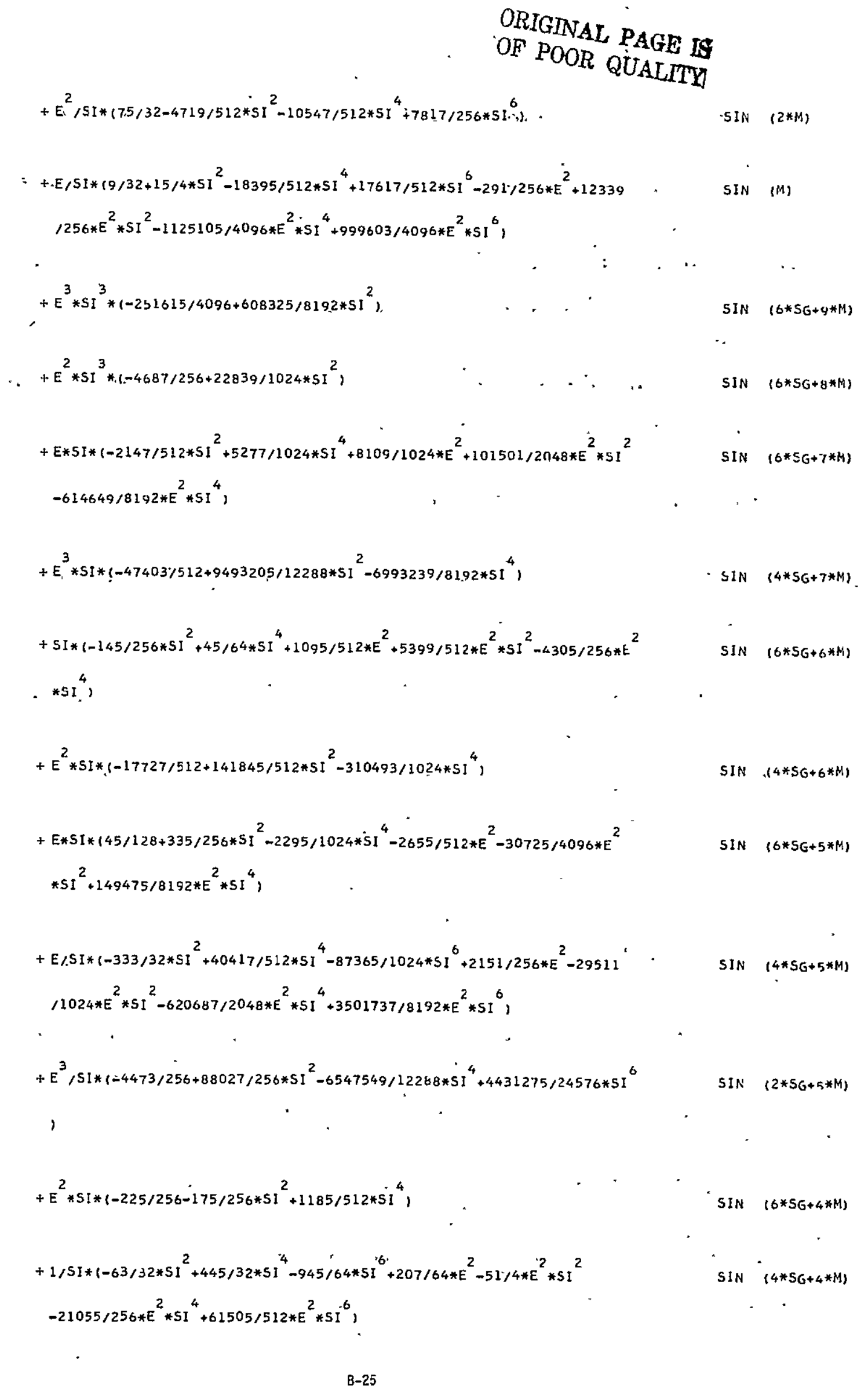




$$
\begin{aligned}
& \text { in } \\
& +E^{2} / S I *\left(-547 / 64+37941 / 256 * S I^{2}-51289 / 256 * S I^{4}+23401 / 512 * 5 I^{6}\right), . \quad \text { SIN }(2 * 56+4 * M) \\
& +E^{3} * S I *\left(675 / 1024-315 / 2048 * 5 I^{2}-6975 / 8192 * 5 I^{4}\right) \quad \text {. . . } \quad \sin (6 * S G+3 * M) \\
& +E / S I *\left(27 / 32-405 / 128 * S I^{2}-4473 / 256 * 5 I^{4}+25685 / 1024 * S I^{6}-27 / 32 * E^{2}, \quad S I N \quad(4 * 56+3 * M)^{-}\right. \\
& +13311 / 512 * E^{2} * S I^{2}-509395 / 4096 * E^{2} * S I^{4}+85653 b / 8192 * E^{2} * 5 I^{6} \text {, } \\
& +E / S I *\left(-117 / 32+1671 / 32 * S I^{2}-28863 / 512 * 51^{4}+793 / 1024 * S I^{6}-5385 / 256 * E^{2} \quad \text { SIN }(2 * S G+3 * M)\right. \\
& +11481 / 256 * E^{2} * 51^{2}+61003 / 512 * E^{2} * 5 I^{4}-1311789 / 8192 * E^{2} * 5 I^{6} \text {, } \\
& +E^{2} / 5 i *\left(-27 / 32+1365 / 128 * S I^{2}-4833 / 128 * S I^{4}+30555 / 1024 * S I^{6}\right) \quad \text { SiN }(4 * S G+2 * H) \\
& +-1 / 51 * 1-9 / 8+93 / 8 * 5 I^{2}-2273 / 256 * S 1^{4}-455 / 128 * 51^{6}-69 / 8 * E^{2}+5715 / 512 * E^{2} \quad \text { SIN }(2 * 5 G+2 * M) \\
& * 5 I^{2}+28935 / 512 * E^{2} * 5 I^{4}-8237 / 128 * E^{2} * S I^{6} \text {, } \\
& +E^{3} / S I *\left\{-297 / 256+10779 / 1024 * 5 I^{2}-86413 / 6144 * S I^{4}+28375 / 8192 * 5 I^{6}\right) \quad \text { SIN }(4 * S G+M) \\
& +E / S I *\left(9 / 32-759 / 32 * 5 I^{2}+4383 / 64 * S I^{4}-47755 / 1024 * S I^{6}+3735 / 256 * E^{2} \quad \text { SIN }(2 * S G+H)\right.
\end{aligned}
$$

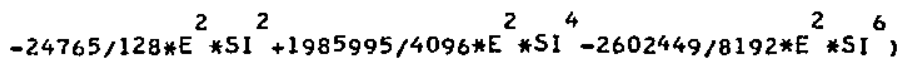

$$
\begin{aligned}
& \text { - } \quad \frac{\mathrm{L}^{10}}{\mathrm{~J}_{2} \mathrm{~J}_{3}} \Delta_{3}(\mathrm{e} \sin . g)=E^{3} / S I *\left(-1587 / 256+26553 / 512 * 5 I^{2}-239357 / 3072 * 5 I^{4}+797765 / 24576 * 5 I^{6},\right. \\
& +E^{2} / S I *\left(-477 / 64+8475 / 128 * S I^{2}-275211 / 2048 * S I^{4}+80399 / 1024 * S I^{6}\right) \\
& \cos (2 * 56) \\
& +E^{3} / 51 *\left(1515 / 256+9189 / 256 * 5 I^{2}-409085 / 4096 * 5 I^{4}+120481 / 2048 * 5 I^{6}\right), \quad \cos (3 * M) \\
& +E^{2} / S I *\left(135 / 32+5865 / 512 * 5 I^{2}-18021 / 512 * S I^{4}+9 B 49 / 512 * S I^{6}, . \quad \cos (2 * M)\right. \\
& +E / S I *\left(117 / 32-687 / 32 * S I^{2}+23601 / 512 * 5 I^{4}-7689 / 256 * S I^{6}+3567 / 256 * E^{2} \quad\right. \text { Cos (il) } \\
& -39621 / 256 * E^{2} * S I^{2}+1480389 / 4096 * E^{2} * 5 I^{4}-475689 / 2048 * E^{2} * 51^{6} \text {, }
\end{aligned}
$$




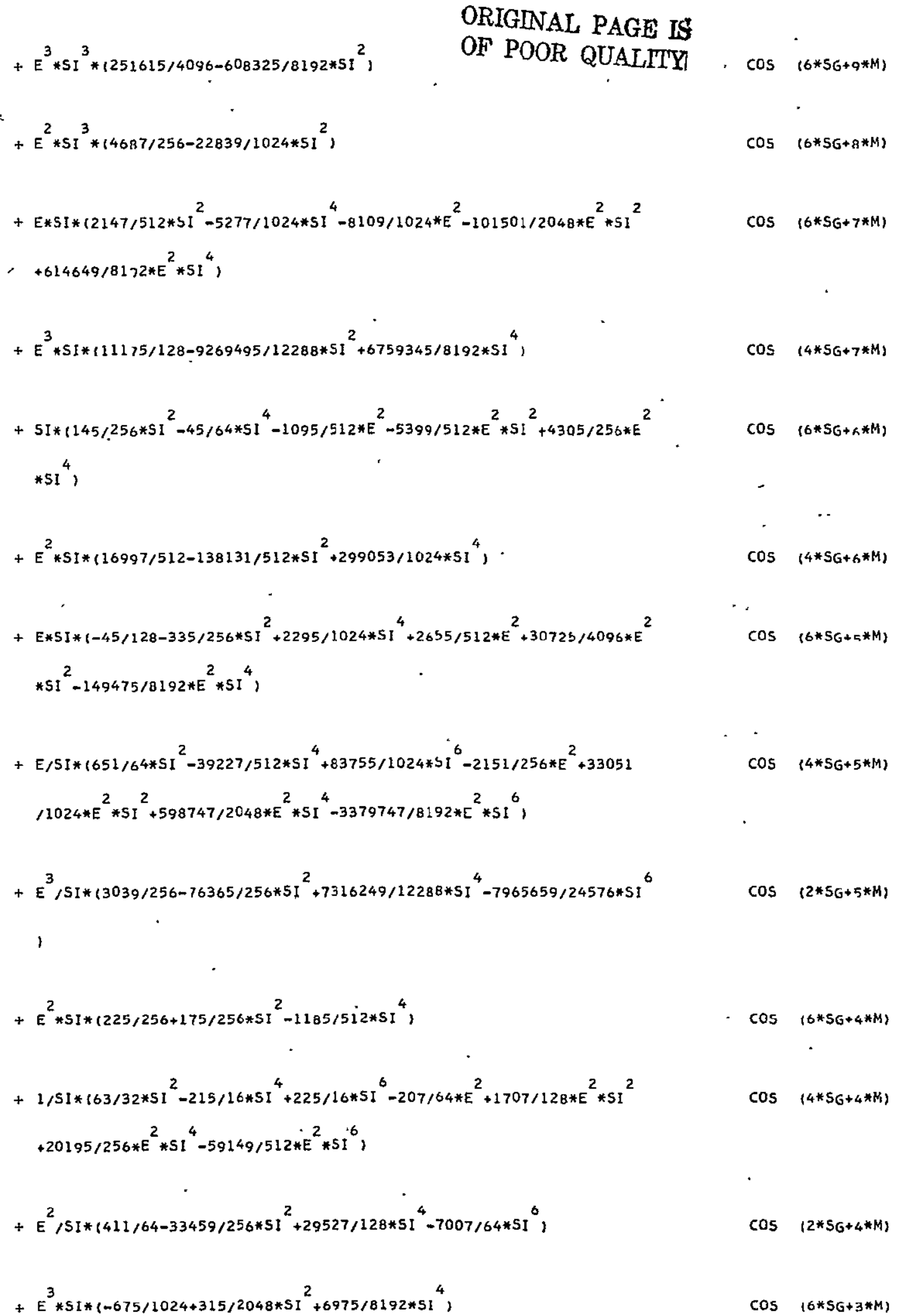


$+E / S I *\left(-27 / 32+405 / 128 * S I^{2}+4293 / 256 * S I^{4}-24695 / 1024 * S I^{6}+27 / 32 * E^{2}-423\right.$

$\cos (4 * 5 G+3 * *)$ $116 * E^{2} * S I^{2}+512905 / 4096 * E^{2} * S I^{4}-864113 / 8192 * E^{2} * S I^{6}$,

$+\varepsilon / S I *\left(99 / 32-1491 / 32 * S I^{2}+34515 / 512 * S I^{4}-24065 / 1024 * 5 I^{6}+5529 / 256 * \varepsilon^{2}\right.$

$\cos (2 * 5 G+3 * M)$ $-5241 / 128 * E^{2} * S^{2}-55819 / 512 * E^{2} * S I^{4}+1196465 / 3192 * E^{2} * S I^{6}$,

$+E^{2} / 5 I *\left(27 / 32-1365 / 128 * S I^{2}+4863 / 128 * S I^{4}-30855 / 1024 * S I^{6}\right.$,

$\cos \left(4 * 56+2 *\left(N_{1}\right)\right.$

$+1 / S I * 19 / 8-81 / 8 * S I^{2}+2641 / 256 * S I^{4}-65 / 64 * 5 I^{6}+147 / 16 * E^{2}-3231 / 512 * E^{2}$

$\cos (2 * S G+2 * M)$ $* S I^{2}-28389 / 512 * E^{2} * S I^{4}+14551 / 256 * E^{2} * S I^{6}$,

$+E^{3} / 51 *\left(297 / 256-10779 / 1024 * 5 I^{2}+86353 / 6144 * 5 I^{4}-28285 / 8192 * S I^{6}\right)$

$\cos (4 * 5 G+N)$

$+E / S I *\left(-9 / 32+429 / 16 * S I^{2}-4455 / 64 * S I^{4}+45175 / 1024 * 5 I^{6}-3537 / 256 * E^{2}\right.$

$\cos (2 * S G+M)$ $+48357 / 256 * E^{2} * S I^{2}-1824383 / 4096 * E^{2} * S I^{4}+2281713 / 8192 * E^{2} * S I^{6}$,

$+1 / S I *\left(9 / 8-405 / 32 * S I^{2}+461 / 16 * 5 I^{4}-18405 / 1024 * 5 I^{6}+405 / 64 * E^{2}-12671\right.$ $\cos (0)$ $128 * E^{2} * S I^{2}+463689 / 2048 * E^{2} * 5 I^{4}-70999 / 512 * E^{2} * 5 I^{6}$,

$\frac{L^{10}}{J_{2} J_{3}} \Delta_{3}(l+g)=E^{2} / S I *\left(-165 / 32+1593 / 64 * 5 I^{2}+43187 / 1024 * 5 I^{4}-277925 / 4096 * 5 I^{6}\right.$, $\cos (S G-M)$ $+E^{3} / S I *\left(-15 / 16+763 / 64 * S I^{2}+146683 / 3072 * S I^{4}-16405 / 256 * S I^{6}\right)$ $\cos (5 G-2 * * i)$ $+E^{3} / S I *\left(-27 / 64+1575 / 1024 * S I^{2}+315 / 256 * 5 I^{4}-10875 / 4096 * S I^{6}\right)$ $\cos (3 * 56)$ $+E / 5 I *\left(-81 / 16+737 / 32 * 5 I^{2}-33877 / 1024 * 5 I^{4}+32505 / 2048 * 5 I^{6}-453 / 16 * E^{2}\right.$ $\cos (56)$ $+133835 / 1024 * E^{2} * 5 I^{2}-3123 / 16 * E^{2} * S 1^{4}+793645 / 8192 * E^{2} * S I^{6}$, $+E^{3} * S I *\left(-15739 / 512+11221 / 3072 * 5 I^{2}+1037179 / 12288 * S I^{4}\right)$ 


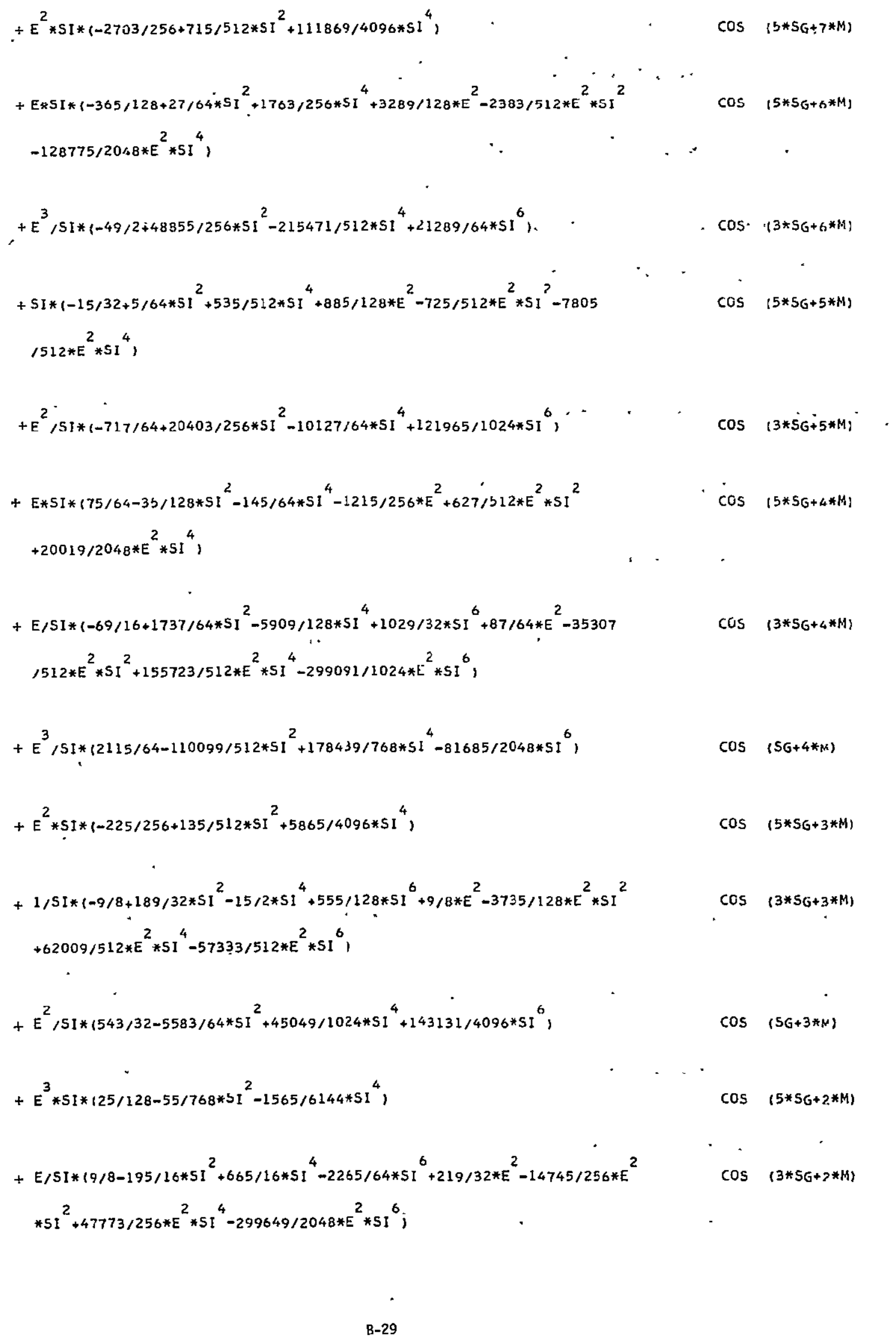




$$
\begin{aligned}
& +E / S I *\left(27 / 4-1959 / 128 * 5 I^{2}-6047 / 128 * S I^{4}+15827 / 256 * S I^{6}+69 / 32 * E^{2}+19965 \quad \cos \quad(5 G+2 *(4)\right. \\
& 1128 * E^{2} * S_{I^{2}}^{2}-616881 / 1024 * E^{2} * S I^{4}+969275 / 2048 * E^{2} * S I^{6}, \\
& +E^{2} / S I *\left(99 / 64-4059 / 256 * 5 I^{2}+29911 / 512 * 5 I^{4}-1515 / 32 * 5 I^{6}\right) \quad \cos (3 * 5 G+(1) \\
& +1 / S I *\left(87 / 4 * S I^{2}-9785 / 128 * S I^{4}+29445 / 512 * S I^{6}-189 / 16 * E^{2}+11697 / 64 * t^{2} \quad \cos \cdot(5 G+M)\right. \\
& * S I^{2}-17159 / 32 * E^{2} * S^{4}+98051 / 256 * E^{2} * S I^{6}, \\
& \frac{\mathrm{L}^{10}}{\mathrm{~J}_{2} \mathrm{~J}_{3}} \Delta_{3} \mathrm{~h}=\mathrm{CI} * \mathrm{E}^{2} / S I *\left(165 / 32+27 / 64 * \mathrm{SI}^{2}-22355 / 1024 * 5 I^{4}\right) \\
& +C I * F^{3} / S I *\left(15 / 16+781 / 128 * S^{2}-64675 / 3072 * S I^{4}\right) \\
& +C I * E^{3} / 5 I *\left(77 / 64-1359 / 1024 * S I^{2}-1695 / 2048 * 5 I^{4}\right) \\
& \div C I * F / S I *\left(81 / 16-193 / 16 * S I^{2}+7685 / 1024 * S I^{4}+453 / 16 * E^{2}-70059 / 1024 * E^{2} \quad \operatorname{COS} \quad(S G)\right. \\
& * 51^{2}+90773 / 2048 * E^{2} * S 1^{4} \\
& +C I * E^{3} * 5 I *\left(15739 / 512+8999 / 768 * S I^{2}\right) \\
& +C I * L^{2} * S I *\left(2703 / 256+497 / 128 * S I^{2}\right) \\
& +C I * E * 5 I *\left(365 / 128+257 / 256 * 5 I^{2}-3289 / 128 * E^{2}-4195 / 512 * E^{2} * S I^{2}, \quad \text { cos }(5 * S G+6 * M)\right. \\
& +C I * E^{3} / S I *\left(49 / 2-45719 / 256 * S I^{2}+141469 / 1024 * 51^{4}\right) \quad \cos (3 * 5 G+6 * M) \\
& +C I * S I *\left(15 / 32+5 / 32 * S I^{2}-885 / 128 * E^{2}-1045 / 512 * E^{2} * S I^{2}, \quad \text { COS }(5 * S G * 5 * M)\right. \\
& +C I * E^{2} / S 1 * 1717 / 64-18969 / 256 * 5 I^{2}+55395 / 1024 * S^{4}, \quad \text { COS }(3 * S G+5 * M) \\
& +C I * E * S I *\left(-75 / 64-5 / 16 * S I^{2}+1215 / 256 * E^{2}+147 / 128 * E^{2} * S I^{2}, \quad \cos (5 * 5 G+4 * A)\right. \\
& \text { B-30. }
\end{aligned}
$$




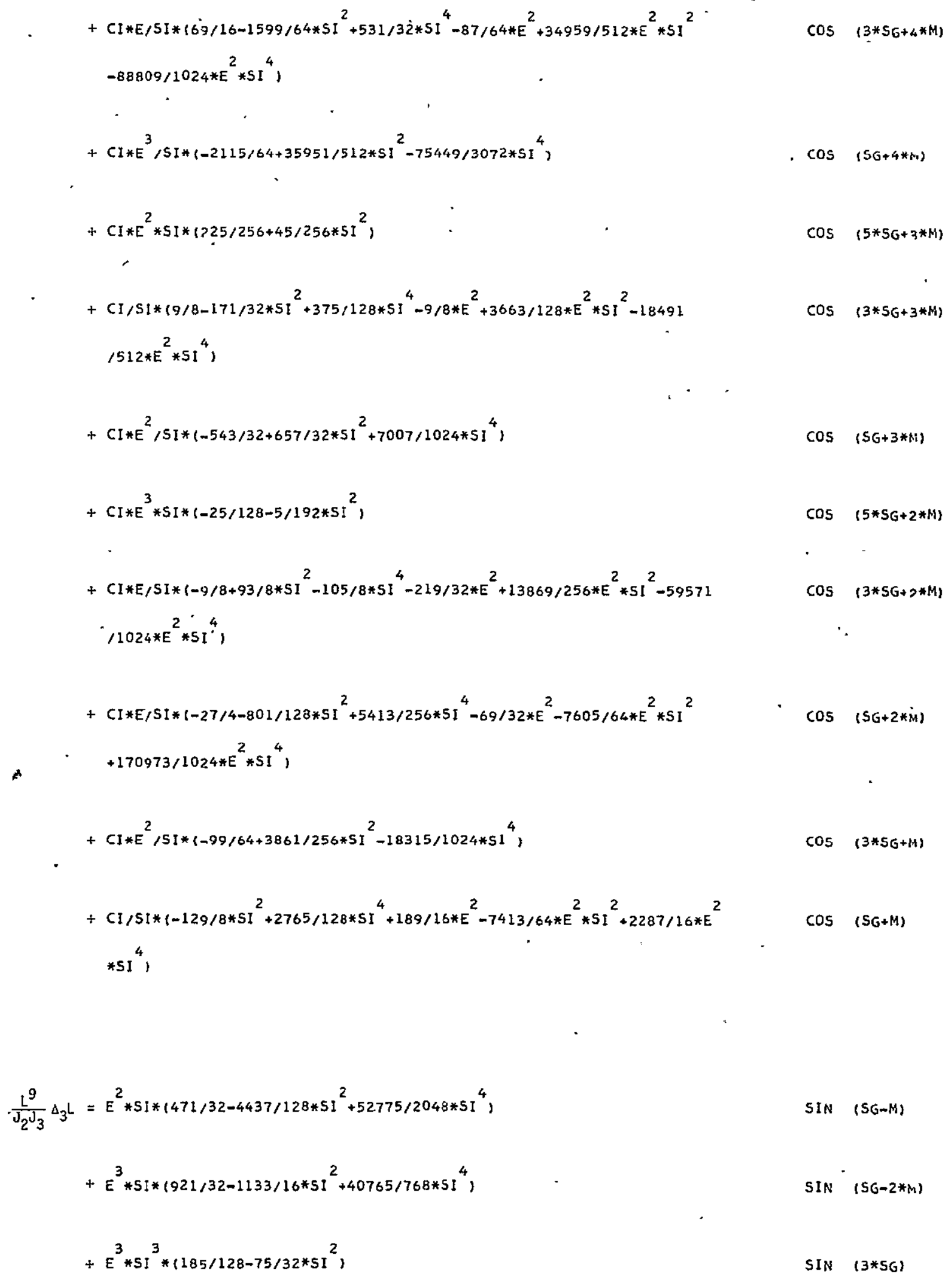




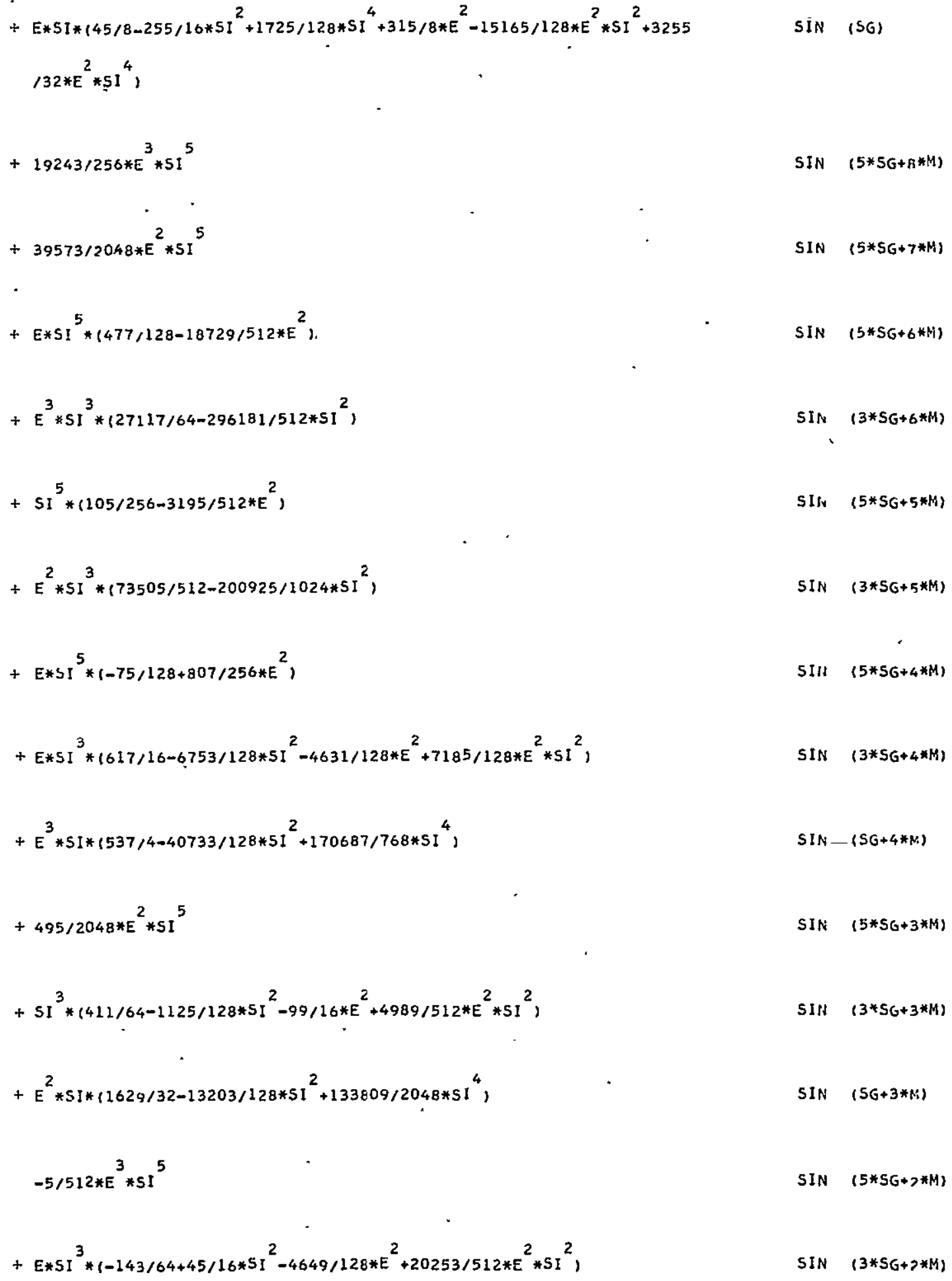


$+E * S I *(117 / 8)-1293 / 64 * 51^{2}+1121 / 128 * 51^{4}+1245 / 32 * E^{2}-5589 / 128 * E^{2} * S I^{2}$

$+E^{2} * S I^{3} *\left\{-5041 / 512+11115 / 1024 * S I^{2}\right\}$

$\div S I *\left(9 / 4-3 / 16 * 5 I^{2}-415 / 256 * S I^{4}+117 / 8 * E^{2}-1203 / 64 * E^{2} * S I^{2}+199 / 16 * E^{2} * S I^{4}\right)$
SIN $(S G+2 * M)$

$\operatorname{SIN}(3 * S G+M)$

$\operatorname{SIN}(S G+M)$

B. 4 Third-Order Short-Period Perturbations due to $\mathrm{J}_{2} \mathrm{~J}_{4}$

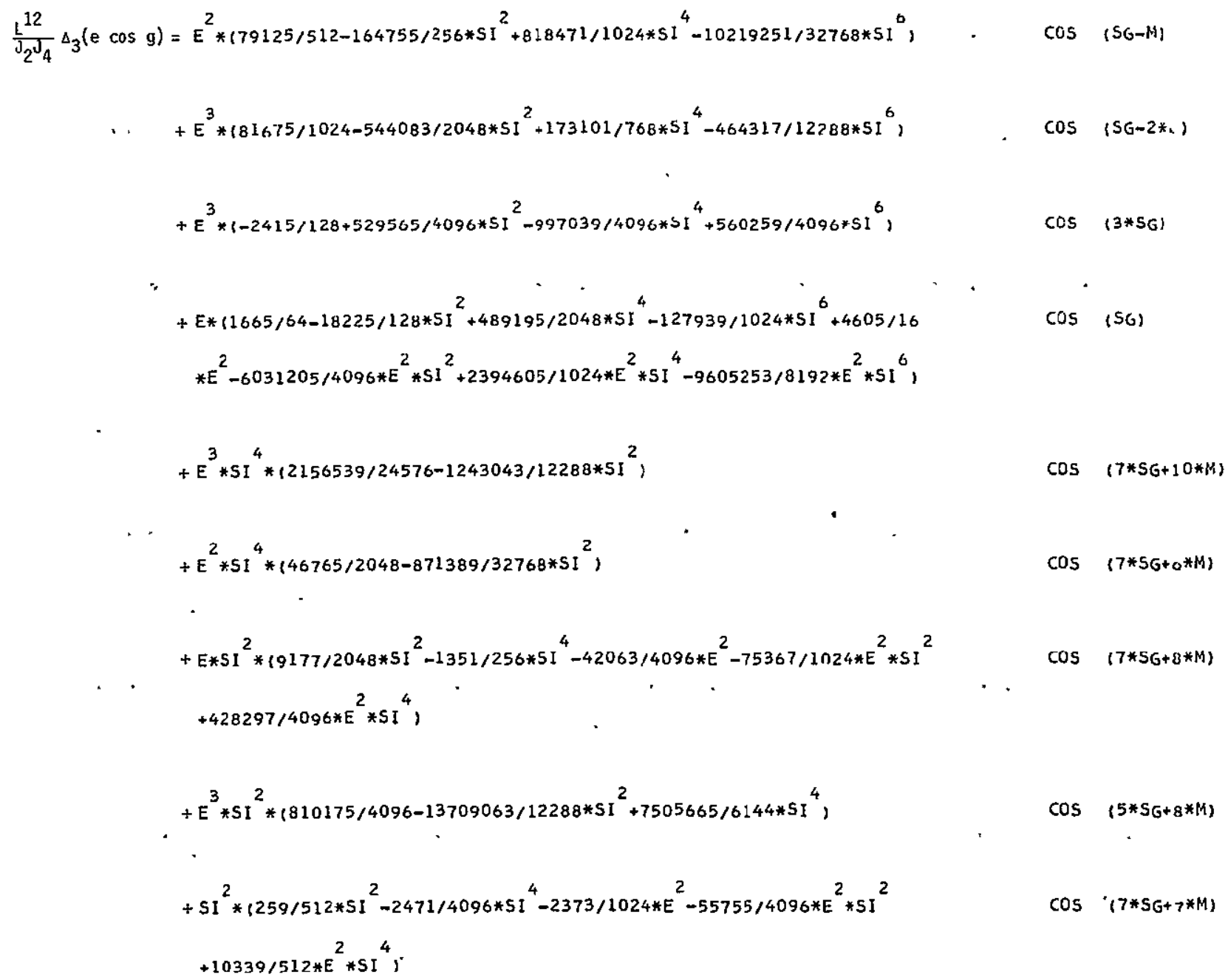




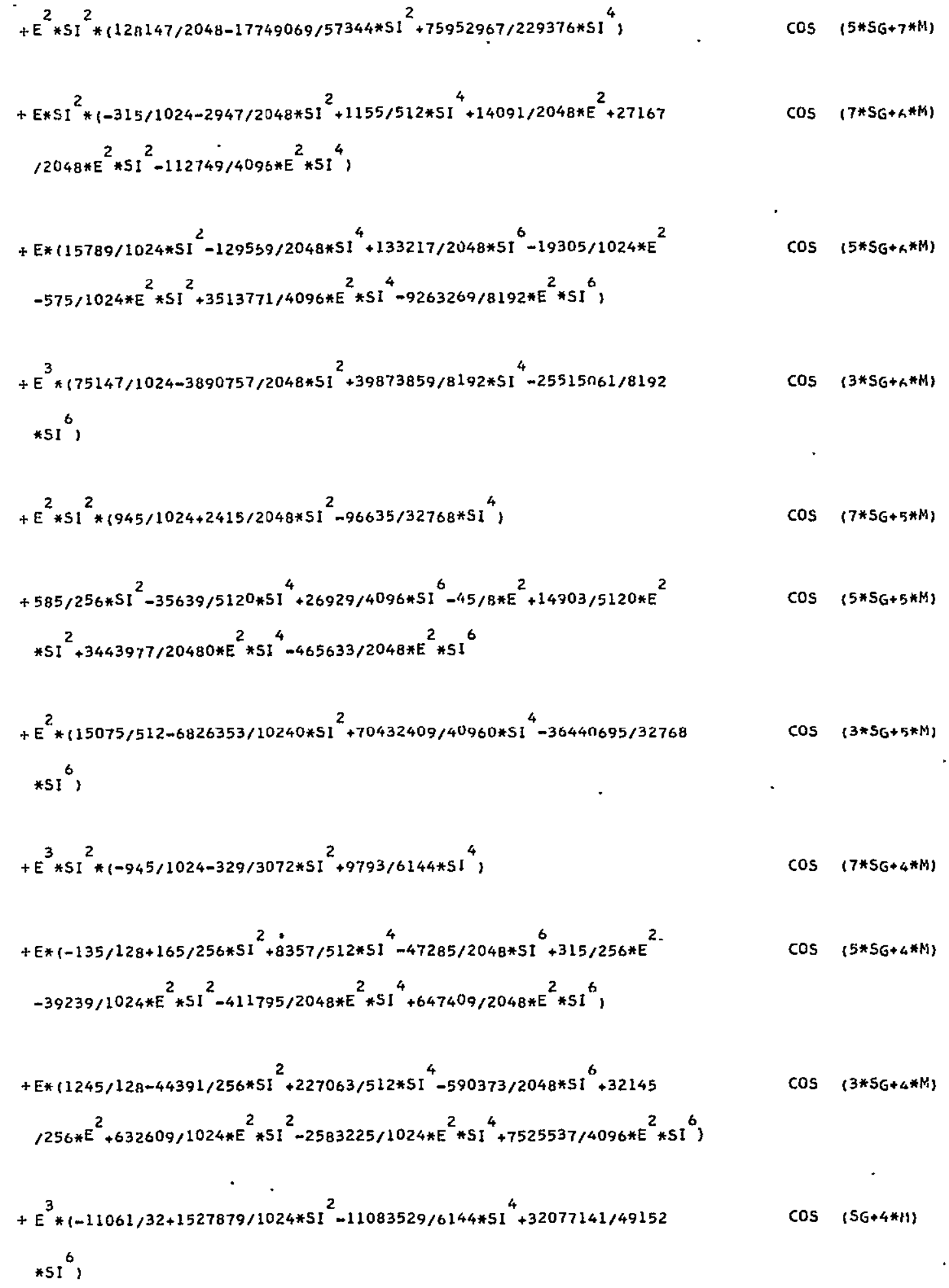




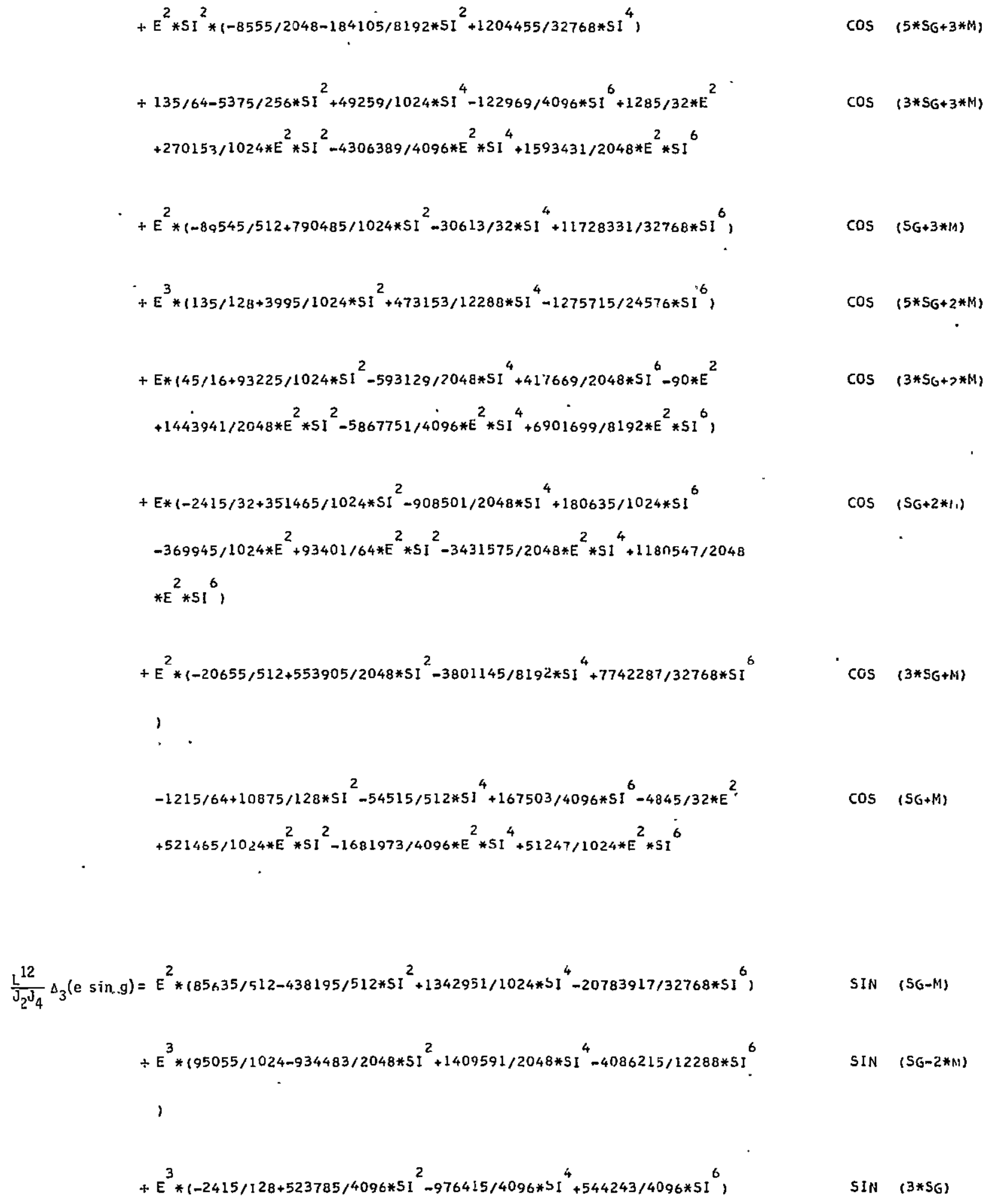




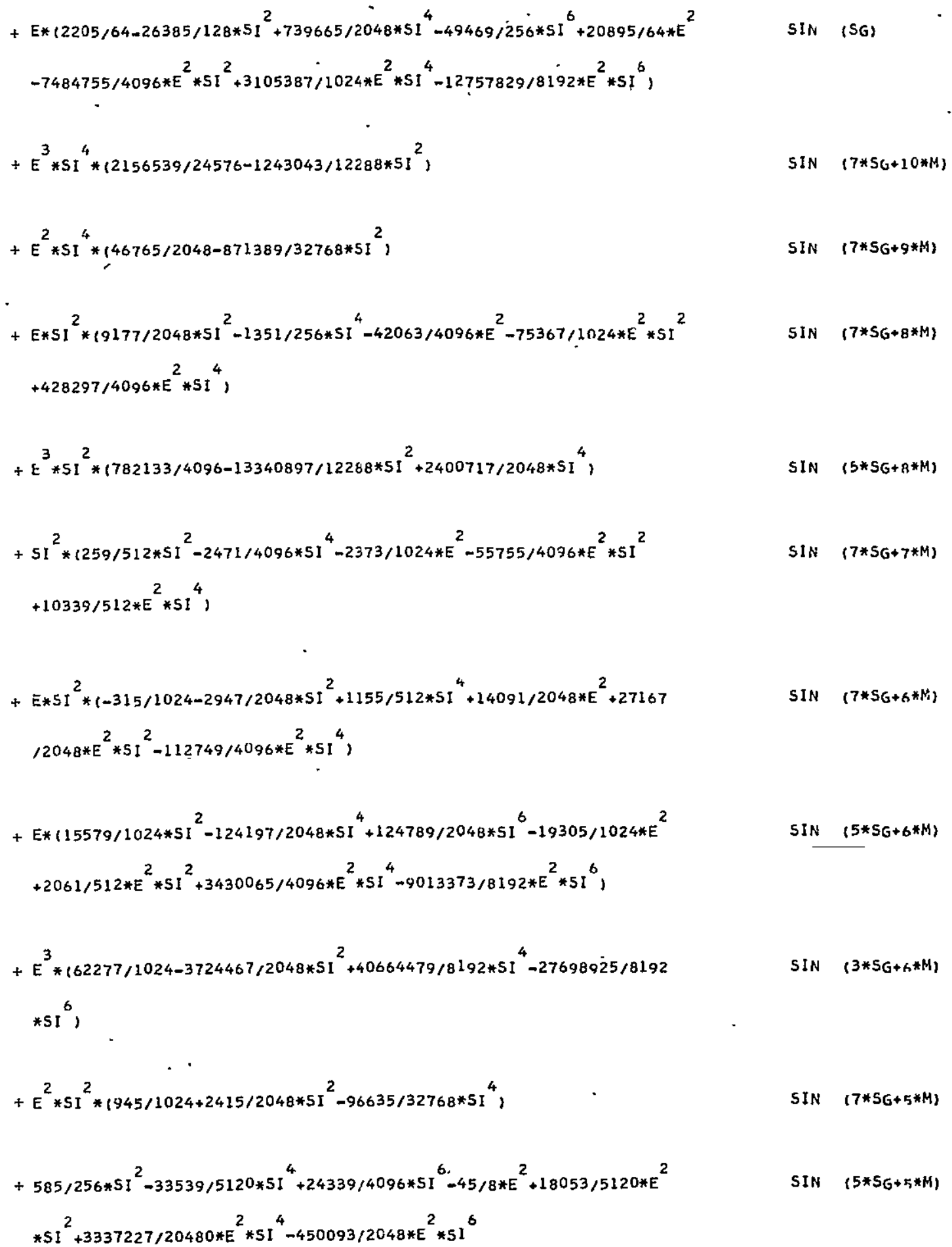




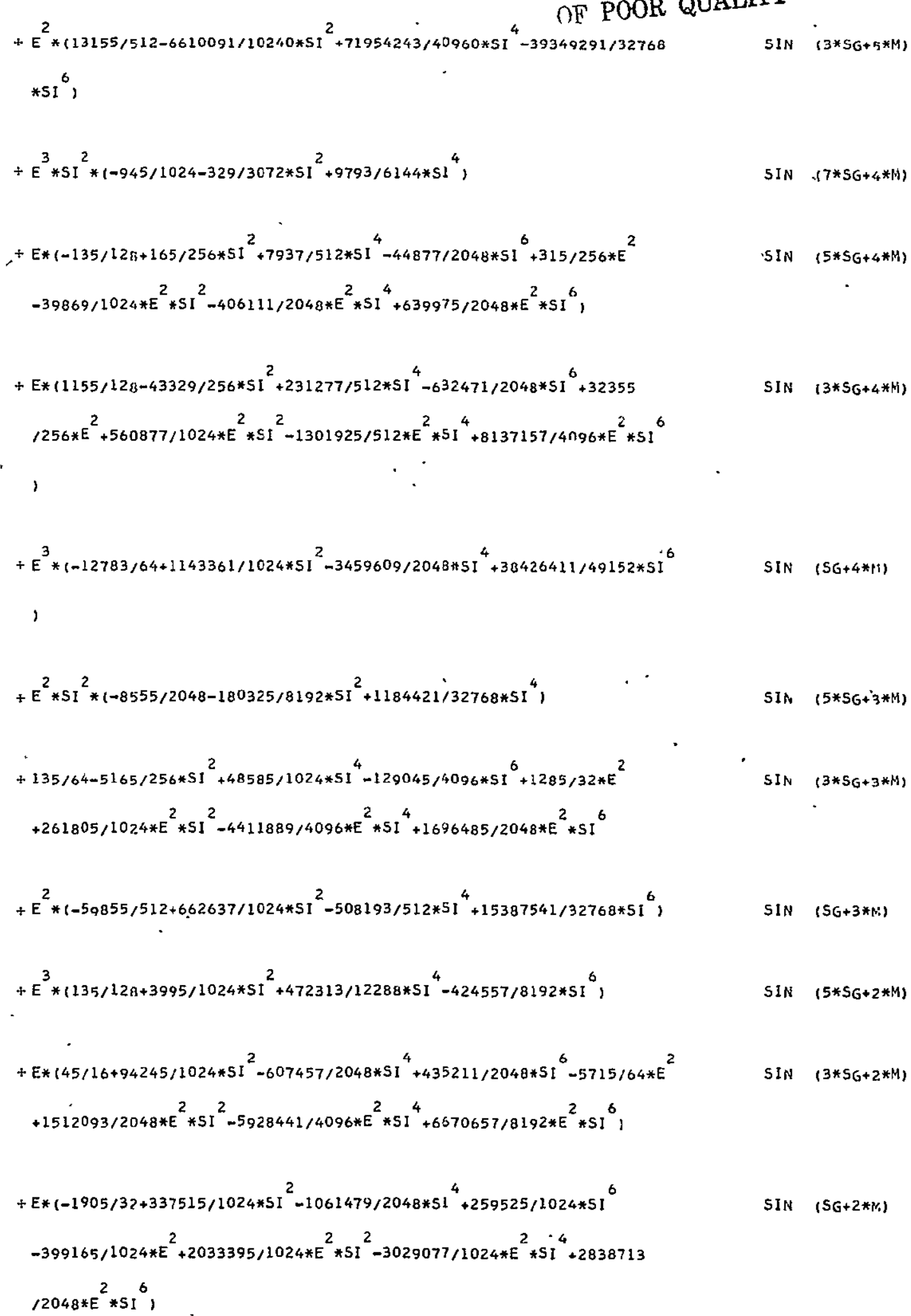




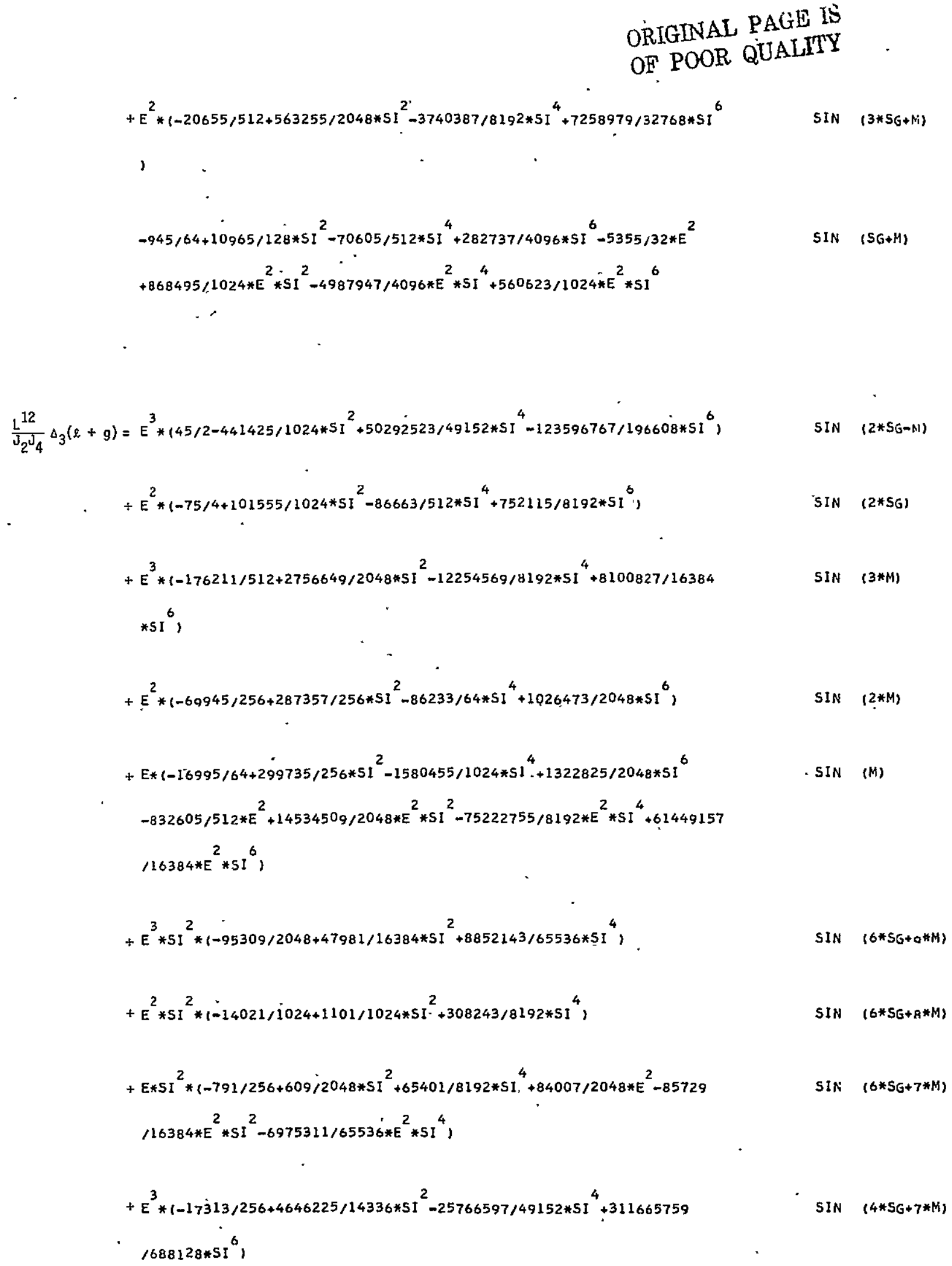


$+S I^{2} *,-105 / 256+49 / 1024 * 5 I^{2}+2023 / 2048 * S I^{4 *}+4697 / 512 * E^{2}-357 / 256 * E^{2} * 5 I^{2}$ $-5579 / 256 * E^{2} * 5 I^{4}$,

$+E^{2} *\left(-6435 / 256+12639 / 128 * 5 I^{2}-106895 / 1024 * 5 I^{4}+348487 / 4096 * 5 I\right.$

$+E^{\prime} * S I^{2} *\left(315 / 256-455 / 2048 * S I^{2}-21525 / 8192 * S I^{4}-18235 / 2048 * E^{2}+29995\right.$ $116384 * E^{2} * 5 I^{2}+1281035 / 65536 * E^{2} * S I^{4}$,

$+E *\left(-15 / 2+27489 / 1280 * S I^{2}+1681 / 10240 * 5 I^{4}-10325 / 4096 * 5 I^{6}+2685 / 256 * E^{2}\right.$ $-156971 / 512 * E^{2} * S^{2}+96626583 / 81920 * E^{2} * S^{4}-36019327 / 32768 * E^{2} * S I^{6}$, ,

$+\dot{E}^{3} *\left(126939,320-2631909 / 1024 * S^{2}+1144303303 / 245760 * S I^{4}-499521881\right.$ $/ 196608 * \mathrm{SI}^{6}$,

$+E^{2} * S I^{2} *\left(-315 / 256+77 / 256 * S I^{2}+18697 / 8192 * S I^{4}\right)$ $-45 / 32+135 / 64 * 5 I^{2}+3659 / 512 * 5 I^{4}-6769 / 1024 * 5 I^{6}+105 / 64 * E^{2}-18885 / 256$ $* E^{2} * S I^{2}+9119 / 32 * E^{2} * S I^{4}-136479 / 512 * E^{2} * S I^{6}$

$+E^{2} *\left(2715 / 16-530281 / 512 * 51^{2}+1887375 / 1024 * 5 I^{4}-8206471 / 8192 * 51^{6}\right)$

$+E^{3} * S I^{2} *\left(945 / 2048-2331 / 16384 * 5 I^{2}-47271 / 65536 * 5 I^{4}\right)$

$+E *\left(-235 / 32 * S I^{2}+62207 / 2048 * S I^{4}-125363 / 4096 * 5 I^{6}-135 / 128 * E^{2}+222505\right.$ $/ 2048 * E^{2} * S^{2}-7359939 / 16384 * E^{2} * S I^{4}+13020707 / 32768 * E^{2} * 5 I^{6}$,

$+E *\left(1715 / 32-9377 / 32 * S I^{2}+1025337 / 2048 * S I^{4}-2201661 / 8192 * 5 I^{6}-35715\right.$ $1256 * E^{2}+868577 / 512 * E^{2} * S I^{2}-61072565 / 16384 * E^{2} * S I^{4}+145906003 / 65536 * E^{2}$. $* S I^{6}$,

$+E^{2} *\left(45 / 32+3535 / 256 * 5 I^{2}-67245 / 1024 * 5 I^{4}+245329 / 4096 * S I^{6}\right)$
$\operatorname{SIN}(6 * S G+6 * M)$

SIN $(4 * S G+G * M)$

SIN $(6 * 5 G+5 * M)$

$\operatorname{SIN}(4 * S G+5 * M)$

SIN $(2 * 56 * 5 * M)$

SIN,$(6 * S G+4 * M)$

SIN. $(4 * S G+4 * M)$

SIN $(2 * 56+4 * M)$

$\operatorname{SIN},(6 * S G+3 * M)$

SIA $(4 * S G * 3 * M)$

$\sin (2 * S G+3 * M)$

$\operatorname{SIN}(4 * S G+2 * M)$ 


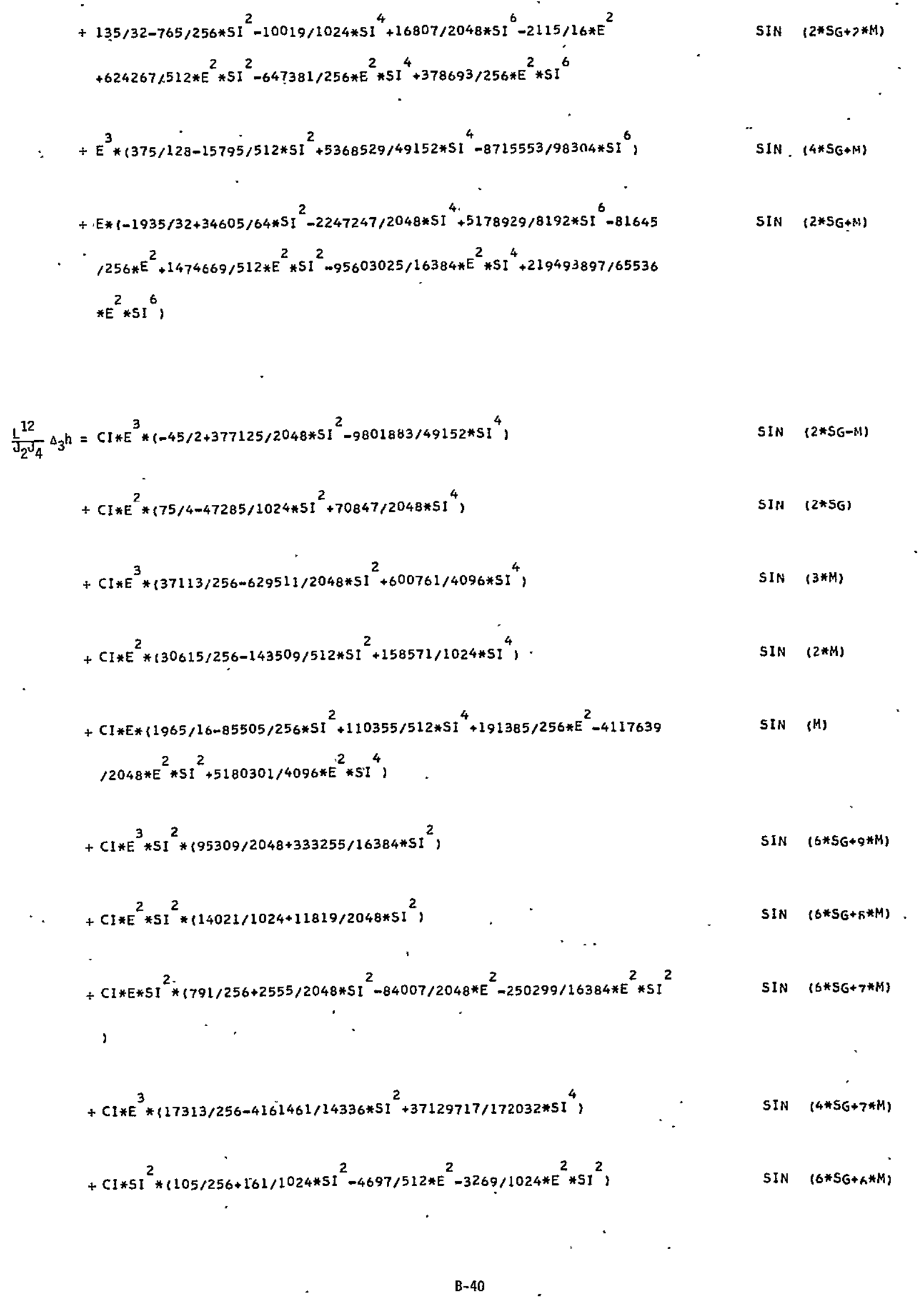


$+C I * E^{2} *\left(6435 / 256-44121 / 512 * S I^{2}+109441 / 2048 * S I^{4}\right)$

$+C I * E * S I^{2} *\left(-315 / 256-805 / 2048 * S I^{2}+18235 / 2048 * E^{2}+42945 / 16384 * E^{2} * S I^{2}\right)$

$+C I * E *\left(15 / 2-22689 / 1280 * S I^{2}+6251 / 1024 * 5 I^{4}-2685 / 256 * E^{2}+77143 / 256 * E^{2}\right.$ $* S I^{2}-2978867 / 8192 * E^{2} * S I^{4}$,

$+C I * E^{3} *\left(-126939 / 320+12321417 / 10240 * 5 I^{2}-41966339 / 49152 * 5 I^{4},\right.$.

$+C I * E^{2} * S I^{2} *\left\{315 / 256+161 / 512 * 5 I^{2}\right.$,

$+C I *\left(45 / 32-45 / 32 * 5 I^{2}-623 / 512 * 5 I^{4}-105 / 64 * E^{2}+18675 / 256 * E^{2} * S I^{2}-1463\right.$ $116 * E^{2} * S^{4}$,

$+C I * E^{2} *\left(-2715 / 16+129707 / 256 * S^{2}-363979 / 1024 * S I^{4}\right)$

$+C I * E^{3} * S I^{2} *\left(-945 / 2048-1449 / 16384 * 5 I^{2}\right)$

$+C I * E *\left(235 / 32 * S I^{2}-10479 / 1024 * S I^{4}+135 / 12 B * E^{2}-221425 / 2048 * E^{2} * S I^{2}\right.$ $+1049895 / 8192 * E^{2} * 5 I^{4}$,

$+C I * E * 1-1715 / 32+38611 / 256 * S I^{2}-207641 / 2048 * S I^{4}+35715 / 256 * E^{2}-1413941$ $12048 * E^{2} * S^{2}+10366189 / 16384 * E^{2} * S^{4} I^{4}$,

$+C I * E^{2} *\left(-45 / 32-3715 / 256 * 5 I^{2}+40831 / 2048 * 5 I^{4}\right)$

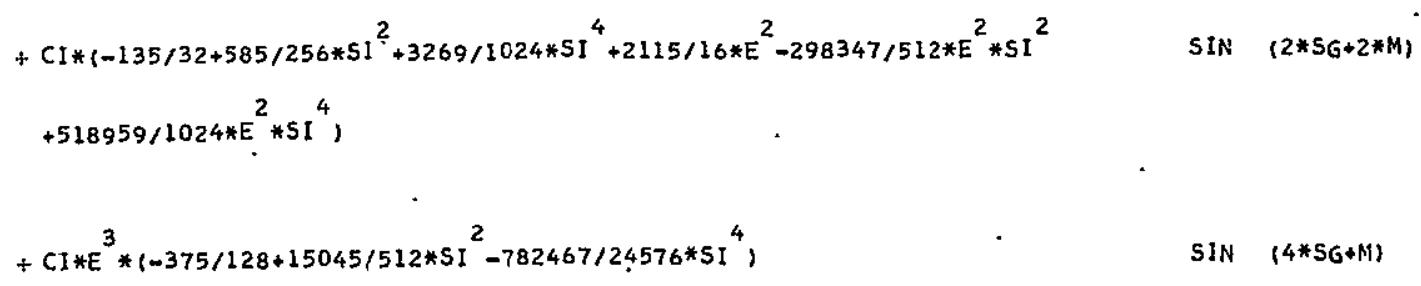

$\sin (4 * 5 G+11)$

$\operatorname{SIN}\{2 * 5 G+5 * M)$

$\sin (6 * 5 G+4 * H)$

$\sin (4 * S G+4 * M)$

$\operatorname{SIN}(2 * S G+4 * M)$

SIN $(6 * S G+3 * M)$

$\sin (4 * 50+3 * M)$

SIN $(2 * S G+3 * M)$

SIN $(4 * S G+2 * M)$

B-41 


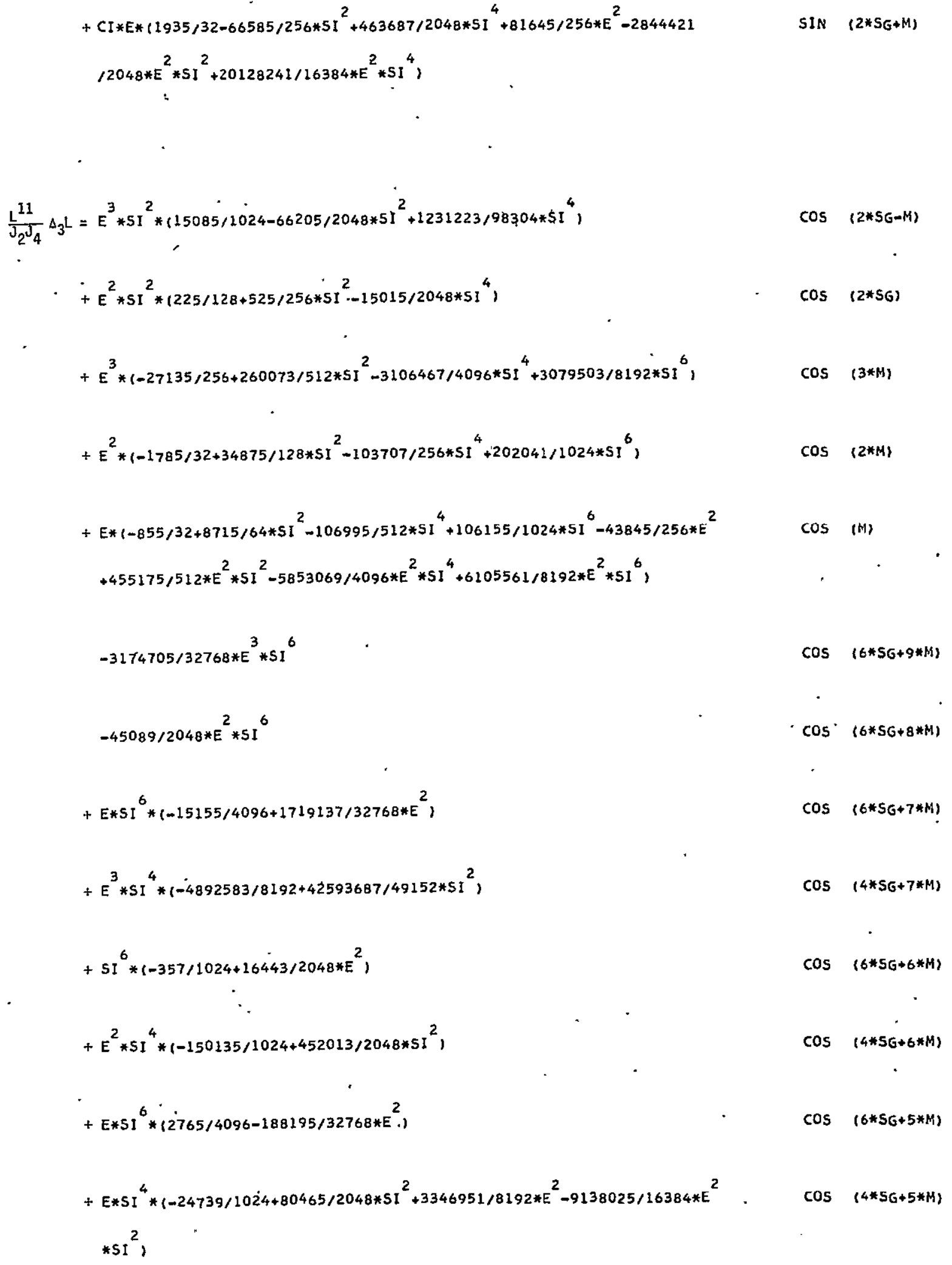




\section{ORIGINAL PAGE IS \\ OF POOR QUALITY}

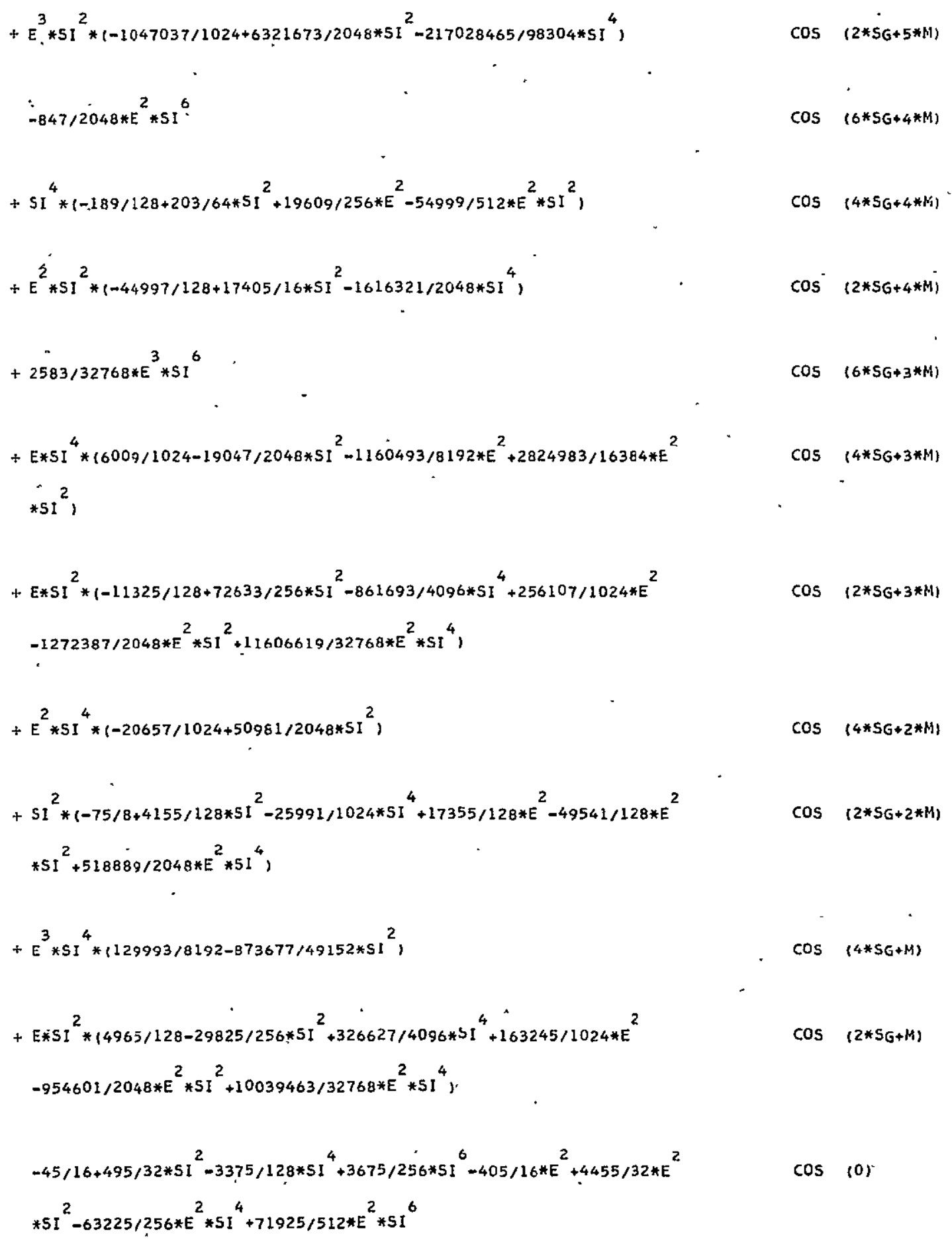




\section{URIGINAL PAGE IS \\ OF POOR QUALITY}

B.5 Second-Order Long-Period Perturbations

$\stackrel{*}{*}(\mathrm{e} \cos g)$

COEFFICIENT OF COS(3*SG)

$1 / A^{2} * A 4^{2} * E^{3} / L^{8} *\left(-375 / 4 / I N G^{3} * 5 I^{2}+18125 / 32 / I N G^{3} * 5 I^{4}-37875 / 32 / I N G^{3} * 5 I^{6}+133875 / 128 / I N G^{3} * S I^{8}-42875 / 128 / 1 N G^{3} * S I^{10}+13 / 8 / I^{2} G^{2}\right.$

$-19075 / 128 / 1 \mathrm{NG}^{2} * S I^{2}+137125 / 256 / 1 \mathrm{NG}^{2} * 5 I^{4}-353325 / 512 / I \mathrm{NG}^{2} * S I^{6}+302575 / 1024 / 1 N \mathrm{G}^{2} * 51^{8}$,

$+1 / A Z^{2} * A 3{ }^{2} * E^{3} \Lambda^{4} *\left(-25 / 16 / I N G{ }^{2} * S I^{2}+25 / 8 / I N G{ }^{2} * S I I^{4}-25 / 16 / I N G{ }^{2} * S I^{6}+5 / 16 / I N G-45 / 32 / I N G * S I^{2}+3 b / 32 / 1 N G * S I^{4}-3 / 32 / 5 I^{2}+1 / 32+1 / 4 * 51^{2}\right.$,

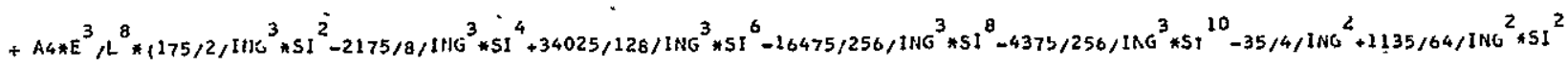
$+14885 / 128 / \mathrm{IGG}^{2} * 5 I^{4}-35065 / 128 / 1 \mathrm{NG}^{2} * 51^{6}+153475 / 1024 / 1 H_{G}{ }^{2} * 5 I^{8}+285 / 32 / 1 N_{G}-725 / 8 / 11 * G * 5 I^{2}+96935 / 512 / I N G * 5 I^{4}-14035 / 128 / I N G * 5 I^{6}$, $+A 2^{2} * E^{3} \mu^{8} * 1585 / 4 / I^{3} v G^{3} * S I^{2}-21065 / 32 / 1 N G^{3} * S I^{4}+141915 / 128 / I N G^{3} * 5 I^{6}-6625 / 8 / I N G^{3} * S I^{8}+29625 / 12 B / I N G^{3} * S I^{10}-117 / B / I N G^{2}+17557 / 12 B$ $/ I N_{G}{ }^{2} * S I^{2}-88465 / 25 S / I N_{G}{ }^{2} * S I^{4}+173625 / 512 / I N N^{2} * S I^{6}-118825 / 1024 / 1 N_{G}{ }^{2} * 5 I^{8}+191 / 32 / I N G-6877 / 128 / I N_{G} * G I^{2}+13073 / 128 / I N_{G} * S I^{4}-56795$ $11024 /$ ING*SI $^{6}$,

COEFFICIENT OF $\operatorname{SIN}(2 * \operatorname{SG})$.

$1 / A^{2}{ }^{2} * A 3 * A 4 * E^{2} / L^{6} * 1-25 / 1 N G^{2} * S I+525 / 4 / I N G^{2} * S I^{3}-775 / 4 / 1 N G^{2} * S I^{5}+175 / 2 / 1 N G^{2} * S I^{7}+5 / 2 / I N G / 51-435 / 8 / I N G * 5 i+1275 / 8 / 1 N G * S 1^{3}-717 b / 64$ IINC*SI ${ }^{5}$

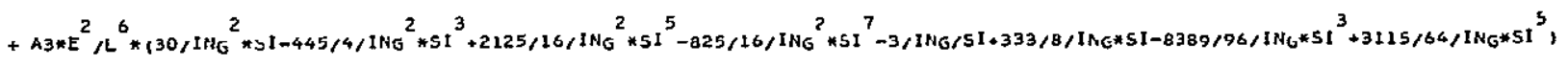

COÉFFICIENT OF COS(SG)

$1 / A 2^{2} * A 4^{2} * E / L^{8} *\left(75 / 4 / I N G^{2} * S I^{2}-11875 / 128 / I N G^{2} * S I^{4}+17675 / 128 / I N G^{2} * S I^{6}-33075 / 512 / 1 N G^{2} * S I^{8}+375 / 4 * E^{2} / 1 N G^{3} * S I^{2}-71375 / 128 * E^{2} / 1 N G^{3}\right.$ ${ }^{*} * S^{4}+73875 / 64 * E^{2} / 1 N G^{3} * S I^{6}-518875 / 512 * E^{2} / I N G^{3} * S I^{8}+165375 / 512 * E^{2} / I N G^{3} * S I^{10}-75 / 8 * E^{2} / I N G^{2}+28525 / 128 * E^{2} / I N G^{2} * S I^{2}-228175 / 256 * E^{2}$ $/ I N G^{2} * S I^{4}+620375 / 512 * E^{2} / I N G^{2} * 5 I^{6}-550025 / 1024 * E^{2} / I N G^{2} * S I^{8}$,

$+1 / A 2^{2} * A 3^{2} * E / L^{4} * 15 / 7 / I N G * S I^{2}-5 / 8 / I N G * S I^{4}+1 / 8-1 / 2 * 5 I^{2}+25 / 16 * E^{2} / I N_{G}{ }^{2} * S I^{2}-25 / A * E^{2} / I N G{ }^{2} * S I^{4}+25 / 1 G * E^{2} / I N G^{2} * S I^{6}-5 / 16 * E^{2} / I N G+65 / 32$ $* E^{2}, I N G * S I^{2}-55 / 32 * E^{2}, I H G * S I^{4}-1 / 32 * E^{2} / 5 I^{2}+19 / 32 * E^{2}-5 / 4 * E^{2} * S I^{2}$,

$+A 4 * E I^{8} * 1-35 / 2 / I N G^{2} * S I^{2}+2555 / 64 / 1 N^{2}{ }^{2} * S I^{4}-1495 / 64 / I N_{G}{ }^{2} * S I^{6}+175 / 256 / I N G{ }^{2} * S I I^{8}+285 / 16 / I N G * S I^{2}-3095 / 64 / I N G * S I^{4}+4025 / 128 / I N G * 5 I^{6}$ $-175 / 2 * E^{2} / I N G^{3} * S I^{2}+18375 / 64 * E^{2} / I N G^{3} * 5 I^{4}-10125 / 32 * E^{2} / I N G^{3} * S I^{6}+30775 / 256 * E^{2} / I N G^{3} * S I^{8}-875 / 256 * E^{2} / N N G^{3} * 5 I^{10} * 35 / 4 * E^{2} / I N G^{2}-34 \theta 5$ $164 * E^{2} / I N G^{2} * S I^{2}-1075 / 16 * E^{2} / 1 N G^{2} * 51^{4}+2435 / 8 * E^{2} / 1 \mathrm{NG}^{2} * S I^{6}-198625 / 1024 * E^{2} / 1 N^{2} * S I^{8}-285 / 32 * E^{2} / I N G+2375 / 16 * E^{2} / I N G * S I^{2}-695 / 2 * E^{2}$ IING*SI ${ }^{4}+54565 / 256 * E^{2}$,ING*SI $I^{6}$ 


\section{ORIGINAL PAGE IS \\ OF POOR QUALITY}

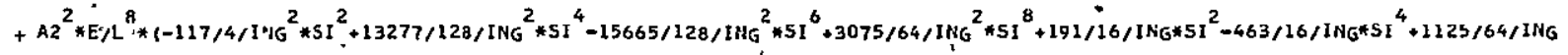

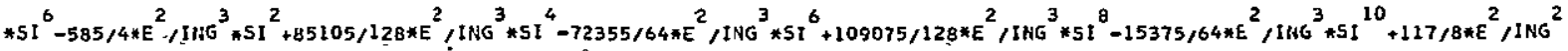

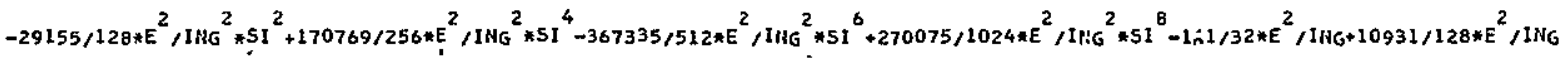
$* S I^{2}-916 B 1 / 512 * E^{2} / I N G * S I^{4}+104865 / 1024 * E^{2} / I H G * S I^{6}$,

$\Delta_{2}^{*}(e \sin g)$ COEFFICIEUT OF SIN(3*5G)

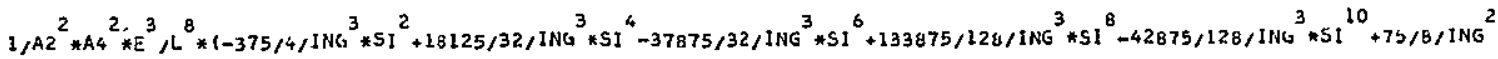

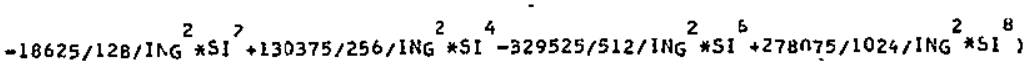

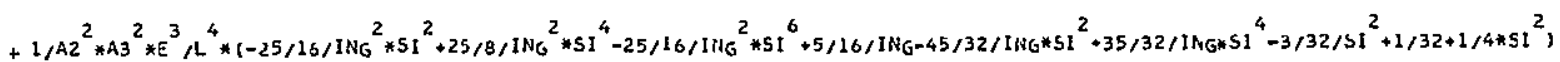

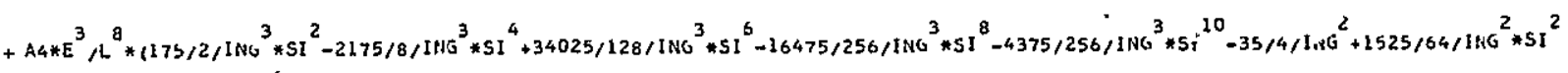

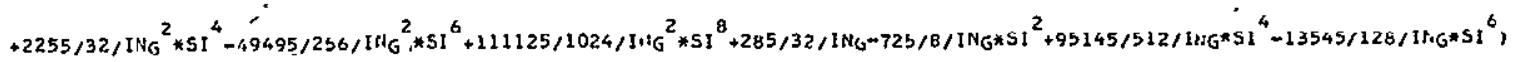

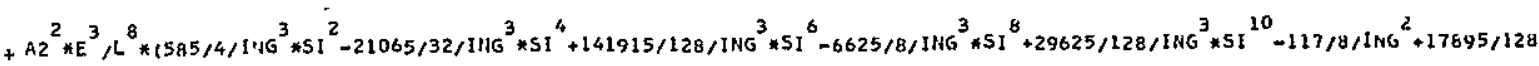

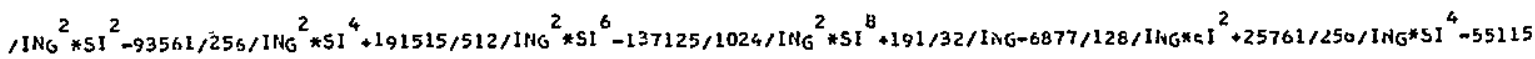
$11024 /$ ING*SI ${ }^{6}$,

COEFFICIENT OF $\cos (2 * 5 G)$

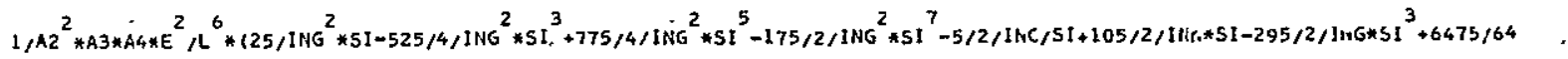
IING*SI ${ }^{5}$,

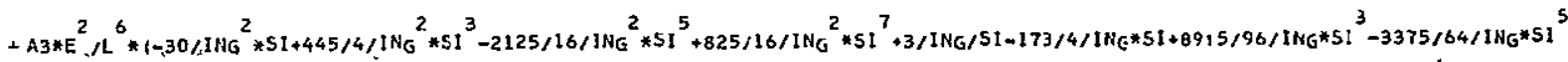
,

COEFF ICIENT OF SIN(SG)

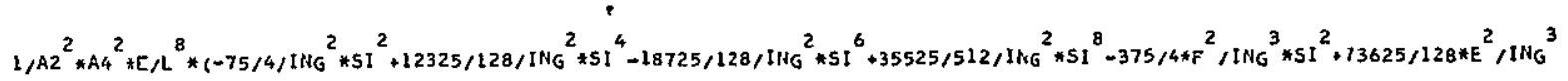

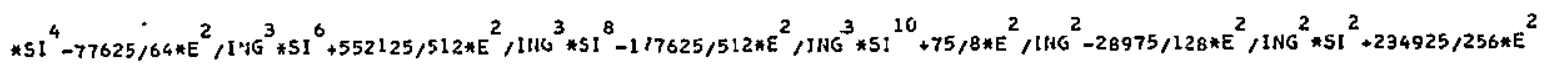

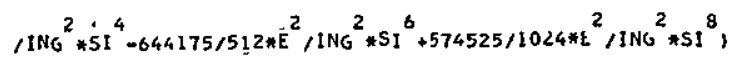

$+1 / A 2^{2} * A 3^{2} * F / L^{4} * 1-5 / 8 / I N G * S I^{2}+5 / 8 / 1 H_{G} * S I^{4}-1 / 8+3 / B * S 1^{2}-25 / 16 * E^{2} / I N_{G}{ }^{2} * S I^{2}+25 / 8 * E^{2} / 1 N_{G}{ }^{2} * S I^{4}-25 / 16 *_{F}{ }^{2} / 1 H_{G}{ }^{2} * S I^{6}+5 / 16 * E^{2} / 1 N G-65 / 32$ $* E^{2} / I N G * S I^{2}+55 / 32 * E^{2} / I N G * 51^{4}-3 / 32 * E^{2} / 5 i^{2}-3 / 32 * E^{2}+5 / 8 * E^{2} * 51^{2}$, 


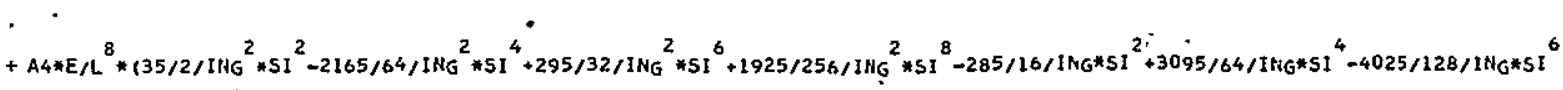
$+175 / 2 * E^{2} / I N G^{3} * S I^{2}-16425 / 64 * E^{2} / I N G^{3} * S I^{4}+13775 / 64 * E^{2} / I N G^{3} * S I^{6}-2175 / 256 * E^{2} / I N G^{3} * S I^{8}-9625 / 256 * E^{2} / I N G^{3} * S I^{10}-35 / 4 * E^{2} / I N \sigma^{2}+3095$ $164 * E^{2}, I N G{ }^{2} * 5 I^{2}+14525 / 128 * E^{2} / 1 N_{G}{ }^{2} * S I^{4}-98845 / 256 * E^{2} / I N G{ }^{2} * 5 I^{6}+241675 / 1024 * E^{2} / I N_{G}{ }^{2} * S I^{8}+285 / 32 * E^{2} / I N G-2375 / 16 * \varepsilon^{2} / 1 N_{G} * 5 I^{2}+695 / 2$ $* E^{2} / I N G * S 1^{4}-54565 / 256 * E^{2}, I N G * 5 I^{6}$,

$+A 2^{2} * E I^{8} *\left(117 / 4 / I N_{G}{ }^{2} * S I^{2}-12939 / 128 / 1 N_{G}{ }^{2} * S I^{4}+14885 / 128 / N^{2}{ }^{2} * S I I^{6}-1425 / 32 / I N G{ }^{2} * 5 I^{8}-191 / 16 / I N G * S I^{2}+463 / 16 / I N G * i I^{4}-1125 / 64 / I N G\right.$

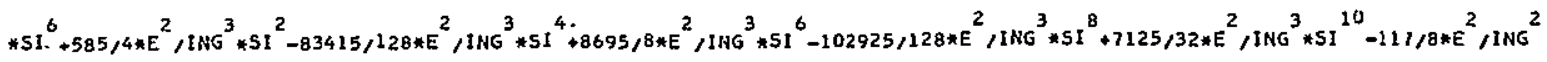
$+28817 / 128 * E^{2} / I N G{ }^{2} * S I^{2}-165569 / 256 * E^{2} / I N G^{2} * S I^{4}+348945 / 512 * E^{2} / I N_{G}{ }^{2} * 5 I^{6}-251175 / 1024 * E^{2} / I N G^{2} * 5 I^{8}+191 / 32 * E^{2} / I N G-10911 / 126 * E^{2} / I N G$ $* \mathrm{SI}^{2}+91681 / 512 * \mathrm{E}^{2} / \mathrm{IHG} \mathrm{HI}^{4}-104865 / 1024 * \mathrm{E}^{2} / I N G * 5 I^{6}$,

COEFFICIENT OF $\cos (0)$.

$1 / A 2^{2} * A 3 * A 4 / L^{6} * 15 / I N G * S I-85 / 4 / I N G * S I^{3}+35 / 2 / I N G * S I^{5}+25 * E^{2} / I N G G^{2} * S 1-525 / 4 * E^{2} / I N G^{2} * S I^{3}+775 / 4 * E^{2} / I N G G^{2} * 5 I^{5}-175 / 2^{2} * E^{2} / I N G^{2} * 51^{7}-5 / 2$ $* E^{2} / I N G / 5 I+1135 / 16 * E^{2} / I N G * 5 I-7125 / 32 * E^{2} / 11 . G * S I^{3}+1295 / 8 * E^{2} / I N G * S 1^{5}$,

$+A 3 / L^{6} *\left(-6 / I H G * S I+65 / 4 / I N G * S I^{3}-165 / 16 / I N G * S I^{5}-30 * E^{2} / I N G{ }^{2} * S I+445 / 4 * E^{2} / I N_{G}{ }^{2} * S I^{3}-2125 / 16 * E^{2} / 1 N G^{2} * S I^{5}+825 / 10 * L^{2} / 1 N_{G}{ }^{2} * 5 I^{7}+3 * E^{2}\right.$

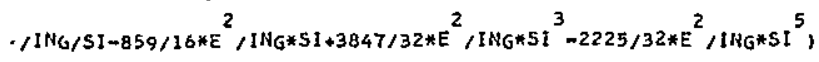

$\Delta_{2}^{*}(\varepsilon+g)$

COEFFICIENT OF COS(3*S(3)

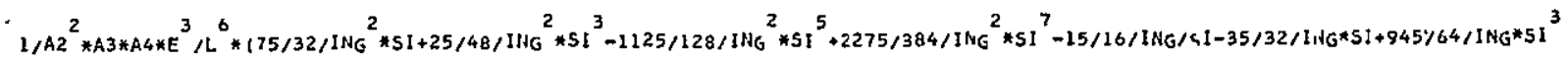
$-5215 / 384 / 1 N G * 5 I^{5}$.

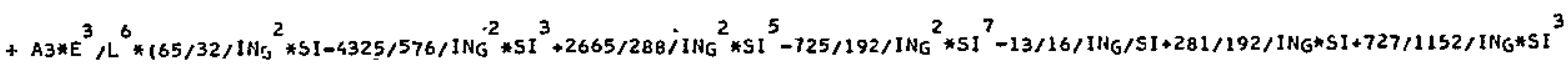
$-1045 / 768 /$ IMG*SI ${ }^{5}$

COEFFICIENT OF SIN $(2 * S G)$

$1 / A 2^{2} * A 4^{2} * E^{2} L^{8} *\left(-375 / 2 / \mathrm{WG}^{3} * 5 I^{2}+18125 / 16 / 1 N G^{3} * S I^{4}-37875 / 16 / I N G^{3} * 51^{6}+133875 / 64 / I N G^{3} * 5 I^{8}-42675 / 64 / 1 M G^{3} * 5 I^{10}+75 / 4 / I N G^{2}-4525\right.$ $116 / 1 N G{ }^{2} * S I^{2}+3975 / 4 / 1 N^{2} * S I^{4}-40425 / 32 / 1 N G G^{2} * 5 I^{6}+8575 / 16 / 1 N G^{2} * S I^{8}, 3$

$+1 / A Z^{2} * A 3^{2} * E^{2} / L^{4} *\left(-25 / 8 / I N G{ }^{2} * S I^{2}+25 / 4 / I N_{G}{ }^{2} * S I^{4}-25 / 8 / I_{G}{ }^{2} * S I I^{6}+5 / 8 / I N_{G}-25 / 8 / I N_{G} * 5 I I^{2}+5 / 2 / I N G * S I^{4}-1 / B / S I^{2}-3 / 16+55 / 64 * 5 I I^{2}\right.$,

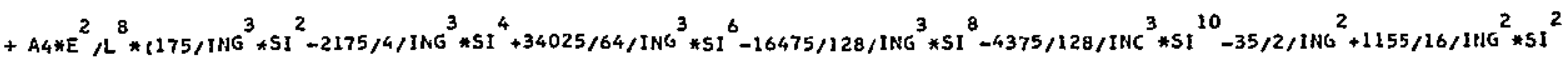
$+7775 / 128 / 1 \mathrm{NG}^{2} * 5 I^{4}-19115 / 64 / I_{G}{ }^{2} * 5 I^{6}+23625 / 128 / I H G_{G}{ }^{2} * 5 I^{8}+285 / 16 / I N G-5945 / 32 / I N_{G} * 5 I^{2}+49215 / 128 / I N G * 5 I^{4}-28175 / 128 / I N G * 5 I^{6}$, $+A Z^{2} * E^{2} L^{8} * 1585 / 2 / 11 G^{3} * S I^{2}-21065 / 16 / 1 N G^{3} * 5 I^{4} ! 141915 / 64 / N^{3}{ }^{3} * 5 I^{6}-6625 / 4 / I N G^{3} * 5 I^{8}+29625 / 64 / 1 N G^{3} * S I^{10}-117 / 4 / 1 N U^{2}+2331 / 8 / 1 N G^{2} * S I^{2}$

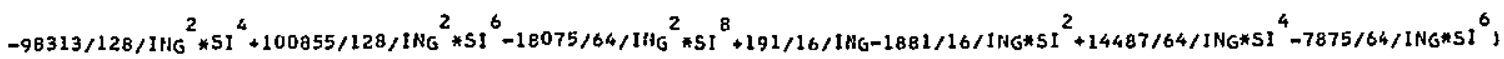




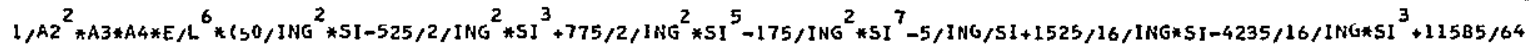

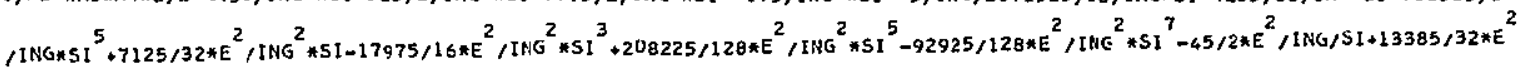

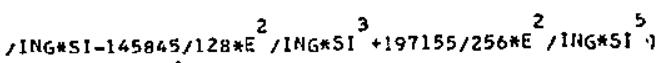

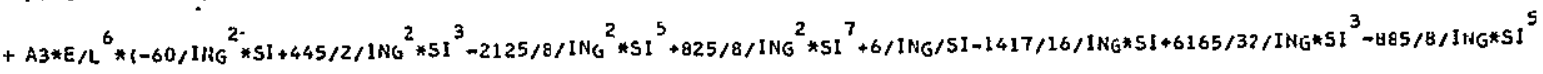

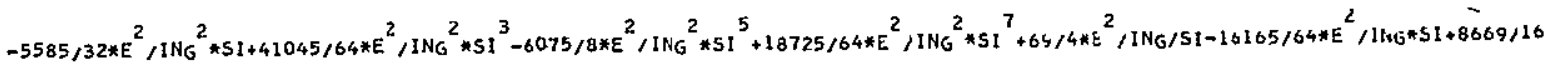
*E ${ }^{2}$ IING*SI ${ }^{3}-78545 / 256 * E^{2}, I N G * S I^{5}$,

$\Delta_{2}^{*}$

COEFFICIENT OF $\operatorname{COS}(3 * 56)$

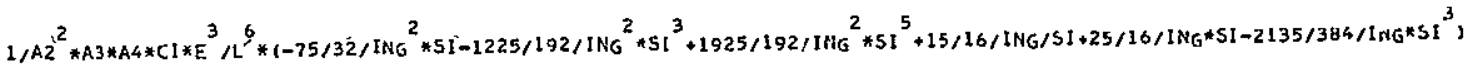

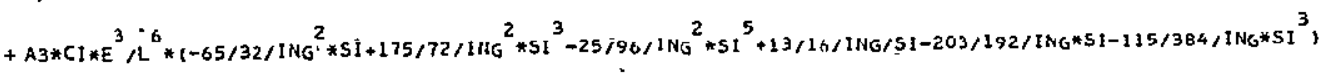

COEFFICIENT OF SIN $2 * 5 G)$

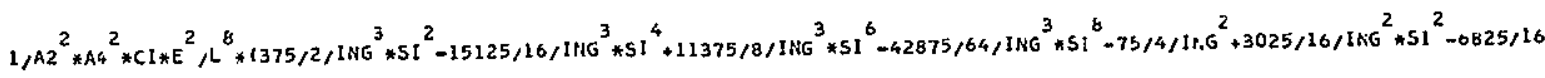
$11 \mathrm{NG}^{2} * \mathrm{SI}^{4}+8575 / 32 / 1 \mathrm{NG}^{2} * \mathrm{SI}^{6}$,

$+1 / A 2^{2} * A 3^{2} * C I * E^{2} / L^{4} *\left(25 / 8 / I \mathrm{HG}^{2} * S I^{2}-25 / 8 / 1 \mathrm{NG}{ }^{2} * S I^{4}-5 / 3 / I N G+5 / 4 / I N G * S I^{2}+1 / 8 / 51^{2}+1 / 4\right)$

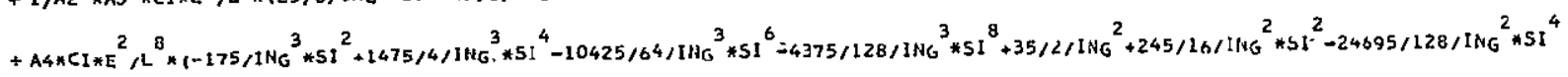
$+21875 / 128 / \mathrm{ING}^{2} * 51^{6}-285 / 16 / 1 \mathrm{NG}+3095 / 32 / 1 \mathrm{NG} * 5 I^{2}-12075 / 128 / \mathrm{IMG}_{\mathrm{G}} * 51^{4}$,

$+A 2^{2} * E\left[* E^{2} / L^{8} *\left(-585 / 2 / \mathrm{NNG}^{3} * 51^{2}+16385 / 16 / 1 \mathrm{NG}^{3} * 51^{4}-76375 / 64 / 1 \mathrm{NG}^{3} * 5 I^{6}+29625 / 64 / 1 \mathrm{NG}^{3} * 5 I^{8} * 117 / 4 / 1 \mathrm{NG}^{2}-1161 / 8 / \mathrm{ING}{ }^{2} * 51^{2} * 27305 / 128\right.\right.$ IING ${ }^{2} * S I^{4}-6225 / 64 / 1 \mathrm{NG}^{2} * S I^{6}-191 / 16 / 1 \mathrm{NG}+463 / 8 / 1 \mathrm{NG} * \mathrm{SI}^{2}{ }^{2}-3375 / 64 / \mathrm{ING}^{2} * 5 I^{4}$,

COEFFICIENT OF $\cos (56)$

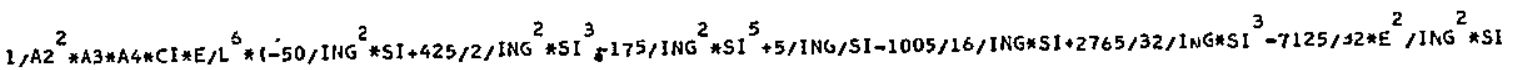

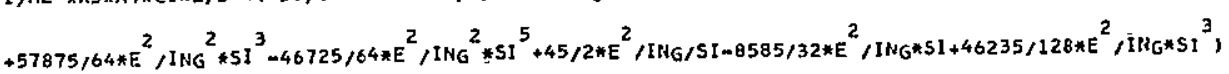

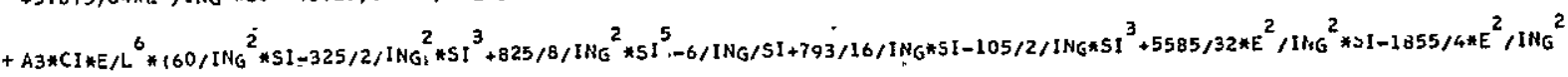
*SI ${ }^{3}+4625 / 16 * E^{2} / I N G^{2} * S 1^{5}-69 / 4 * E^{2} / I N G / 5 I+9077 / 64 * E^{2} / I N G * S I-18935 / 128 * E^{2} / I N G * S I^{3}$, 
$\Delta_{3}^{*}(\mathrm{e} \cos g)$

COEFFICIENT OF SIN $(2 * 5 G)$

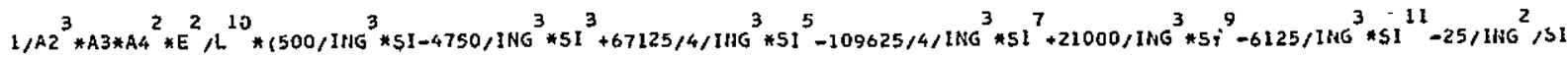
$+2075 / 2 / \mathrm{ING}^{2} * \mathrm{SI}-110925 / 16 / \mathrm{NNG}^{2} * \mathrm{SI}^{3}+281575 / 16 / \mathrm{ING}^{2} * 5 I^{5}-1209075 / 64 / \mathrm{ING}^{2} * \mathrm{SI}^{7}+23152 \mathrm{~b} / 32 / 1 \mathrm{NG}^{2} * 5 I^{9}$,

$+1 / A Z^{3} * A 3^{3} * E^{2} L^{6} *\left(-25 / 4 / I N G^{2} * S I^{3}+25 / 2 / I N G^{2} * S I^{5}-25 / 4 / I N G^{2} * S I^{7}+2 b / 8 / I N G * 5 I-365 / 24 / I N G * S I^{3}+145 / 12 / I N G * S I^{5}+1 / 32 / S I * 37 / 16 * 5 I+113\right.$ $124 * 51^{3}$

$+1 / A 2 * A 3 * A G H F{ }^{2} / L^{10} *\left(-1500 / 1 N G^{3} * S 1+11450 / 1 N G^{3} * S I^{3}-259775 / 8 / 1 N G^{3} * 5 I^{5}+1403725 / 32 / 1 N G^{3} * S I^{7}-915775 / 37 / 1 H G^{3} * S 1^{9}+116375 / 16 / 1 N G^{3} * S I^{11}\right.$ $+75 / 1 H_{G}{ }^{2} / 5 I-11175 / 4 / I N_{G}{ }^{2} * S I+1419955 / 96 / I N_{G}{ }^{2} * S I^{3}-11357495 / 384 / I N_{G}{ }^{2} * S I^{5}+9844145 / 384 / I N G^{2} * 51{ }^{7}-8323175 / 1024 / I H_{G}{ }^{2} * S I^{9}+285 / 8 / 1 N G$ $/ 5 I-29925 / 32 / I_{G} * 5 I+434435 / 128 / I N_{G} * S I^{3}-534305 / 128 / I N_{G} * 5 I^{3}+1729595 / 1024 / I N G * S I^{7}$,

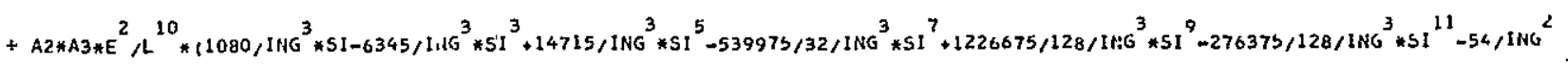

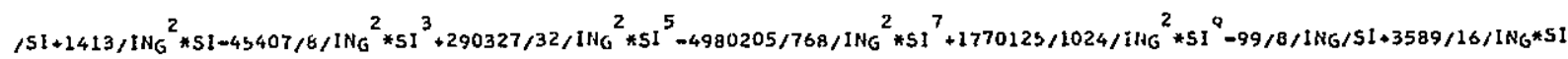
$-141751 / 256 /$ ING*SI $^{3}+482271 / 1024 / I_{H G * S I^{5}}-259525 / 2048 / I H G * 5 I^{7}$,

COEFFICIENT OF COS(SG)

$1 / A^{3} * A 4^{3} * E / L^{12} * 1-375 / 2 / I N G^{3} * S I^{2}+52375 / 32 / 1 H G^{3} * S I^{4}-5581625 / 1024 / 1 N G^{3} * 5 I^{6}+17686575 / 2048 / 1 H G^{3} * 5 I^{6}-26445875 / 4096 / 1 N G^{3} * 5 I^{10}$ $+15735125 / 8192 / \mathrm{ING}^{3} * 5 \mathrm{i}^{12}$,

$+1 / A 2^{3} * A 3^{2} * A 4 * E L^{8} *\left(-25 / 4 / I N_{G}{ }^{2} * 5 I^{2}+2175 / 64 / I N G{ }^{2} * S I^{4}-6525 / 128 / 1 H_{G}{ }^{2} * S I^{6}+2975 / 128 / 1 N_{G}{ }^{2} * S I^{8}-5 / 2 / I N G+1985 / 64 / I N G * S I^{2}-11235 / 128\right.$ /ING*SI ${ }^{4}+15995 / 256 / I N G * 5 I^{6}$,

$+1 / A Z * A 4^{2} * E / L^{12} *\left(10 \angle 5 / 2 / 1 N G^{3} * S I^{2}-113225 / 32 / 1 N G^{3} * S I^{4}+9420375 / 1024 / 1 N G^{3} * S I^{6}-11756325 / 1024 / 1 N G{ }^{3} * S 1^{8}+28463575 / 4096 / 11 N G^{3} * 5 I^{10}\right.$ $-3362625 / 2048 / 1 N G^{3} \cdot * S I^{12}+75 / 16 / I N G^{2} * 5 I^{2}+6175 / 128 / I_{N G}{ }^{2} * S I^{4}-164425 / 512 / 1 M^{2} * S I^{6}+254625 / 512 / I N G^{2} * 5 I^{8}-469175 / 2048 / I N G^{2} * S I^{10}-4725$

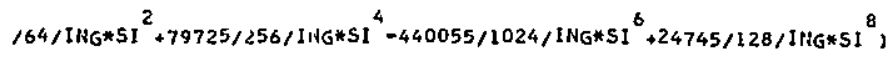

$+1 / A 2 * A 3^{2} * E / L^{8} * 145 / 4 / I N G{ }^{2} * 5 I^{2}-2215 / 64 / 1 N G{ }^{2} * S I^{4}+4515 / 128 / 1 N^{2} * S I^{6}-1525 / 128 / 1 N_{G}{ }^{2} * S I^{8}+3 / I N G-2229 / 64 / I N G * 5 I^{2}+587 / 8 / 1 N G * S I^{4}-10795$ /256/ING*SI ${ }^{6}$,

+A2*A4*E/L ${ }^{12} * 1-45 / 2 / I N \sigma^{3} * S I^{2}-21475 / 32 / I N G^{3} * S I^{4}+34 B 4 B 45 / 1024 / I N G^{3} * S I^{6}-12422185 / 2048 / I N G^{3} * S I^{6}+9683275 / 2048 / 1 N \sigma^{3} * S I^{10}-5621875$

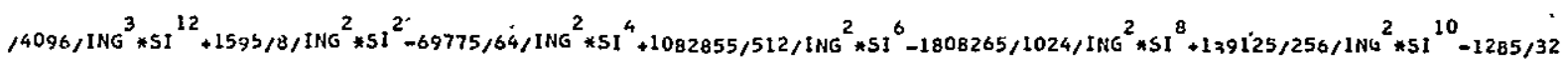
$/ I N_{G} * S I^{2}+63925 / 256 / I N G * 5 I^{4}-867503 / 2048 / I N G * S I^{6}+875665 / 4096 / I N_{G} * S I^{8}$,

$+A 2^{3} * E / L^{12} *\left(-1053 / 2 / 1 N G^{3} * 5 I^{2}+100053 / 32 / 1 \mathrm{NG}^{3} * 5 I^{4}-7602699 / 1024 / 1 \mathrm{NG}^{3} * S I^{6} * 4511495 / 512 / 1 \mathrm{NG}^{3} * S I^{8}-10707875 / 2048 / 1 \mathrm{NG}^{3} * S I^{10}+2538375\right.$ $12048 / \mathrm{ING}^{3} * 5 \mathrm{I}^{12}+1215 / 16 / \mathrm{ING}^{2} * 5 \mathrm{I}^{2}-47777 / 128 / 1 \mathrm{NG}^{2} * 5 \mathrm{I}^{4}+171037 / 256 / \mathrm{ING}^{2} * 5 \mathrm{I}^{6}-538965 / 1024 / \mathrm{ING}^{2} * 5 \mathrm{I}^{8}+80175 / 512 / \mathrm{ING}^{2} * 51^{10}+3815 / 256$ IING*SI ${ }^{2}-7951 / 512 / 1 H G * S I^{4}-52377 / 2048 / 1 N G * 5 I^{6}+210959 / 8192 / I_{N G * S I}{ }^{8}$, 


\section{ORIGINAL PAGE IS OF POOR QUALIIY}

$\Delta_{3}^{*}(e \sin g)$

COEFFICIENT OF $\operatorname{COS(2*SG)}$

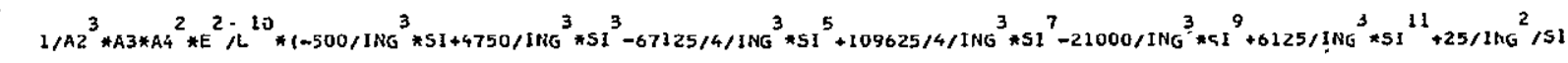
$-1000 / I N G^{2} * S I+837725 / 128 / I N G^{2} * 5 I^{3}-2086325 / 128 / 1 N G^{2} * S I^{5}+8824025 / 512 / I N G^{2} * 5 I^{7}-1609675 / 256 / 1 \mathrm{NG}^{2} * 5 I^{9}$,

$+1 / A 2^{3} * A 3^{3} * E^{2} / L^{6} * 125 / 4 / I N G G^{2} * 5 I^{3}-25 / 2 / 1 N G^{2} * S I^{5}+25 / 4 / I H G^{2} * 5 I^{7}-5 / 2 / 1 N G * 5 I+545 / 48 / 1 / 1 G * S I^{3}-425 / 48 / I N G * 511^{5}-1 / 16 / S I+2 * S I-91 / 24 * S I^{3}$,

$+1 / A 2 * A 3 * A 4 * E^{2} / L^{10} * 11500 / I N G^{3} * S I-11450 / I N G^{3} * 5 I^{3}+259775 / B / 1 H G^{3} * S I^{5}-1403725 / 32 / 1 N G^{3} * S I^{7}+915775 / 32 / 1 N G^{3} * 5 I^{9}-110375 / 16 / I N G^{3} * 511^{11}$

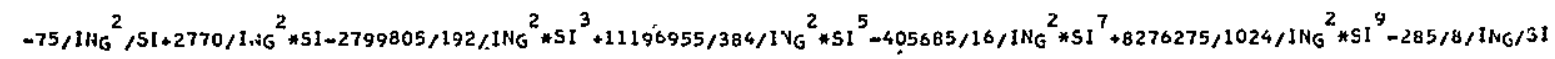

$+30495 / 32 / I N_{G} * 5 I-464585 / 128 / I H_{G} * 5 I^{3}+598473 / 128 / 1 N_{G} * 5 I^{5}-2026325 / 1024 / I H_{G} * 5 I^{7}$ ',

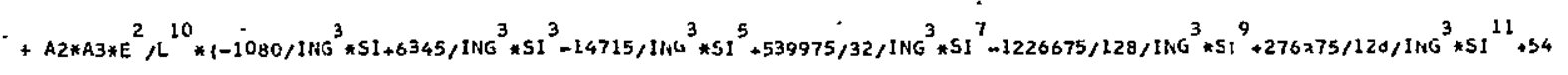
$/ \mathrm{ING}^{2} / 51-5847 / 4 / \mathrm{ING}^{2} * 51+770789 / 128 / \mathrm{ING}^{2} * 51^{3}-3804757 / 384 / \mathrm{NGG}^{2} * 51^{5}+1406045 / 192 / \mathrm{IHG}^{2} * 51^{7}-2077725 / 1024 / 1 \mathrm{NG}^{2} * 51^{3}+99 / 8 / 1 \mathrm{HG} / 5 \mathrm{I}$ $-1699 / 8 /$ ING $5 I+129371 / 256 / 1 N G * 5 I^{3}-420835 / 1024 / 1 N G * S I^{5}+210085 / 2048 /{I N G * S I^{7}}^{7}$,

COEFFICIENT OF Sin(SO)

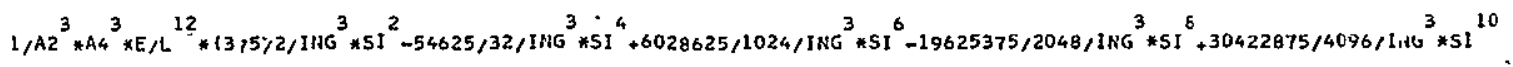
$-18136125 / 8192 /$ ING $^{3} * 51^{12}$

$+1 / A 2^{3} * A 3^{2} * A 4 * E / L^{8} * 125 / 4 / 1 M G^{2} * S I^{2}-2325 / 64 / I N_{G}{ }^{2} * S I^{4}+1175 / 128 / 1 N_{G}{ }^{2} * 5 I^{6}-3325 / 128 / I N G{ }^{2} * S 1^{8}+3 / 2 / I N G-1855 / 64 / I N G * 51^{2}+10155 / 128 / 1 N G$ - $\quad * 51^{4}-14245 / 256 / 1 N G * S I^{6}$,

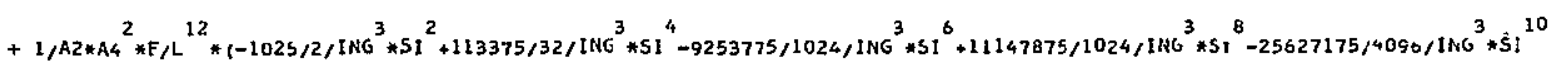

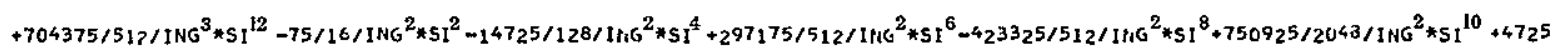

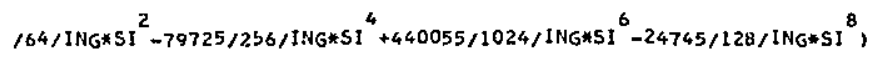

$1+1 / A 2 * A 3^{2} * E / L^{5} * 1-45 / 4 / I N G^{2} * 5 I^{2}+2085 / 64 / I N G{ }^{2} * 5 I^{4}-3955 / 128 / 1 N G{ }^{2} * S I^{6}+1225 / 128 / I N G{ }^{2} * S 1^{8}-3 / 1 N G+2011 / A^{4} / 1 \mathrm{NG} * 5 I^{2}-4055 / 64 / I N G * S I^{4}$ $+9055 / 256 / 1{ }^{\prime} \cdot G * 51^{6}$,

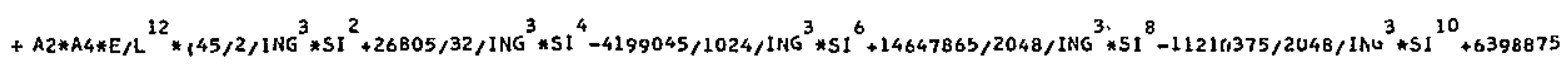

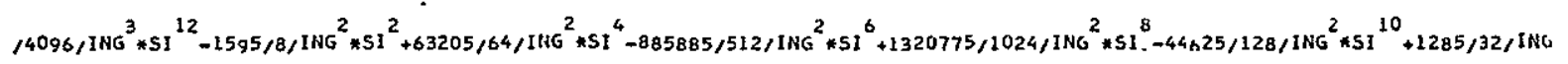
*SI ${ }^{2}-63925 / 256 / I_{\text {NG*SII }}^{4}+867503 / 2048 / I N G * S I^{6}-875665 / 4096 /$ NGG $^{2} I^{8}$,

$+A 2^{3} * E / L^{12} *\left(1053 / 2 / 1 N G^{3} * S I^{2}-97011 / 32 / I H G^{3} * 5 I^{4}+7149571 / 1024 / I N \sigma^{3} * S^{6} I^{6}-1029075 / 128 / I N G^{3} * 5 I^{8} * 9479275 / 2048 / 1 N G^{3} * S I^{10}-2182875 / 2048\right.$ $/ 1 N G^{3} * 5 I^{12}-1215 / 16 / I N G^{2} * 5 I^{2}+42811 / 128 / I N G^{2} * 5 I^{4}-135501 / 256 / N^{2} G^{2} * S I^{6}+369345 / 1024 / 1 N G^{2} * 5 I^{8}-46425 / 512 / 1 N^{2} * 51 I^{1 U}-3815 / 256 / 1 N G * 5 I^{2}$

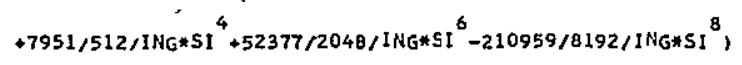


COEFFICIENT OF $\cos (0)$

$1 / A 2^{3} * A 3 * A 4^{2}{ }^{2} L^{10} *\left(-50 / I N G^{2} * S I+425 / I N G^{2} * S I^{3}-10025 / 8 / I N G^{2} * S I^{5}+2975 / 2 / I N G^{2} * S I^{7}-1225 / 2 / I N G^{2} * S I^{9}-500 * E^{2} / I N G^{3} * S I+19375 / 4 * E^{2} / I N G^{3}\right.$

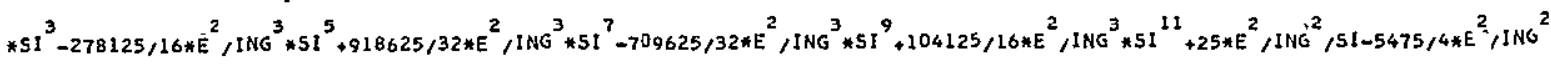

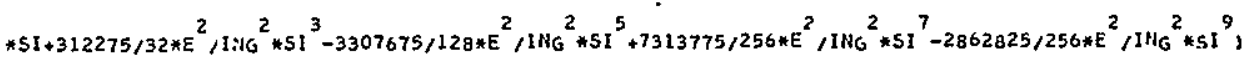

$+1 / A 2^{3} * A 3^{3} / L^{5} * 15 / 4 / I N_{G} * S 1^{3}-5 / 4 / I N G * S I^{5}+3 / 16 * S 1-13 / 16 * S 1^{3}+25 / 4 * E^{2} / I N G{ }^{2} * S 1^{3}-25 / 2 * E^{2} / N_{G}{ }^{2} * S I^{5}+25 / 4 * E E^{2} / I N G{ }^{2} * 51^{7}-65 / 16 * E^{2} / I N G * S I$ $+345 / 16 * E^{2}, I M G * S I^{3}=35 / 2 * E^{2}, I N G * S I^{5}-1 / 16 * E^{2} / S I+51 / 16 * E^{2} * S I-113 / 16 * \dot{E}^{2} * S I^{3}$,

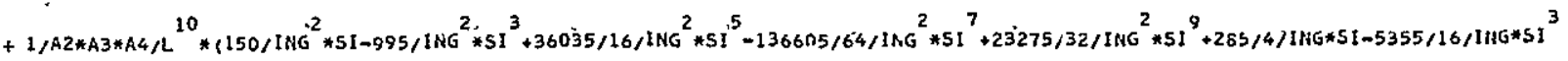

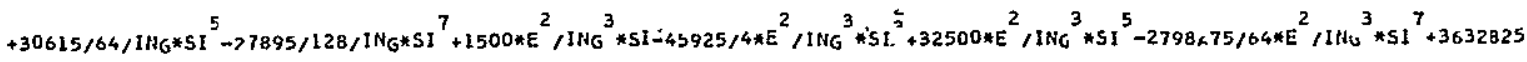

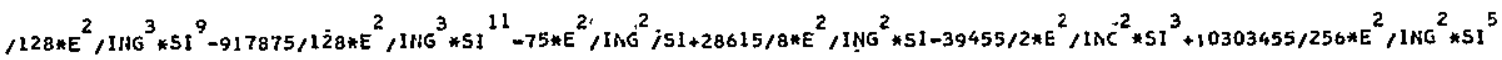
$-18078865 / 512 * E^{2} / I^{1}, G^{2} * 51^{7}+5775875 / 512 * E^{2} / 1 N_{G}{ }^{2} * S 1^{9}-285 / 8 * E^{2} / I N G / S I+20895 / 16 * E^{2} / 1 N_{G} * S 1-328015 / 64 * E^{2} / 1 H_{G} * 51^{3} * 423175 / 64 * E^{2} / I N G$ $* S^{5}-711795 / 256 * E^{2}$, ING*SI $^{7}$,

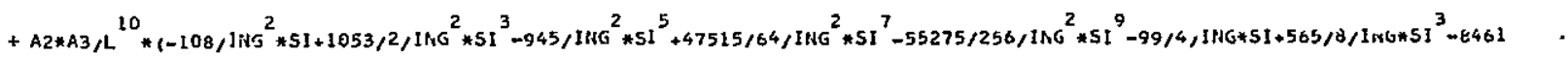

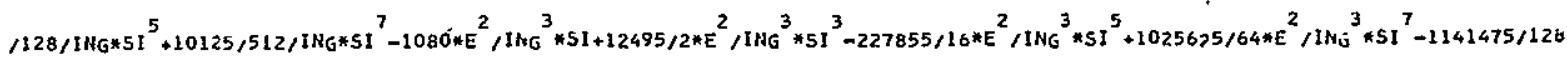

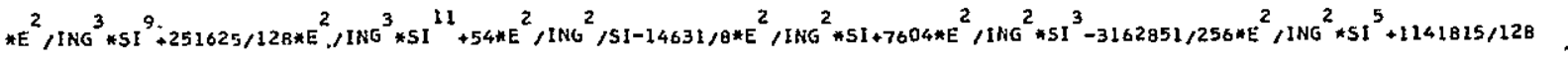

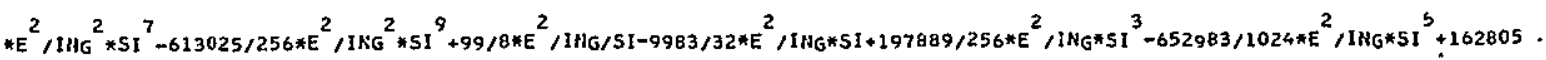
$1024 * E^{2} / I_{G} * S I^{7} 1$

$\cdot$

$\Delta_{3}^{*}(\varepsilon+g)$

COEFFICIENT OF SIN(2*SG)

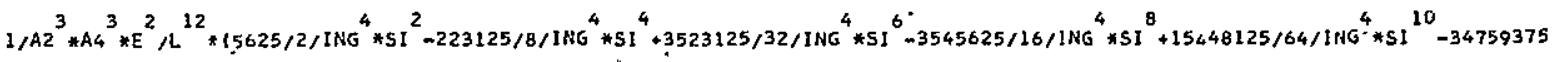

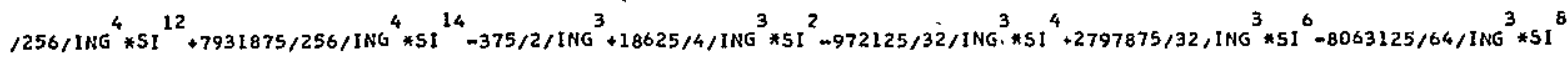
$+11423125 / 128 / \mathrm{MG}^{3} * 51^{10}-1586375 / 64 / \mathrm{ING}^{3} * 5 I^{12}$,

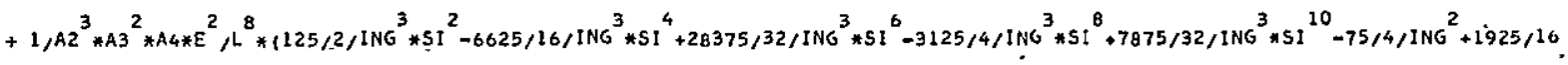

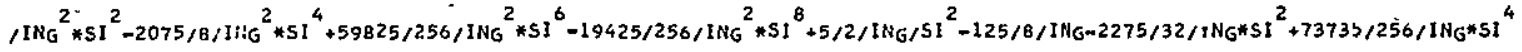
$-27125 / 128 / 1 \mathrm{BNG} * \mathrm{SI}^{\circ}$ !

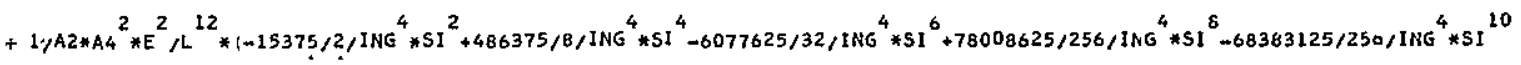

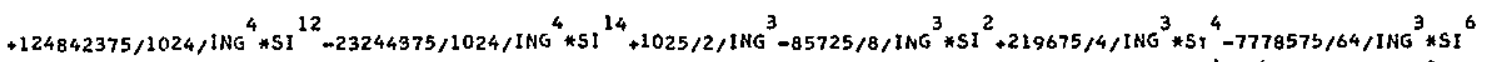

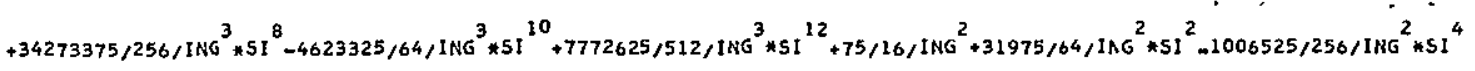
$+10660975 / 1024 / \mathrm{ING}^{2} * \mathrm{SI}^{6}-2873675 / 256 / \mathrm{ING}^{2} * 5 \mathrm{I}^{8}+4345075 / 1024 / \mathrm{IMG}^{2} * \mathrm{SI}^{10}{ }^{10}-4725 / 64 / \mathrm{ING}+145875 / 128 / \mathrm{IMG}_{4} \mathrm{SI}^{2}-3871365 / 1024 / 1 \mathrm{NG} * \mathrm{SI}^{4}$ $+4752335 / 1024 / 1 N G * 5 I^{6}-123725 / 64 /$ ING $^{6} * 5 I^{8}$, 


\section{ORIGINAL PAGE IS \\ OF POOR QUALIIY}

$+1 / A 2 * A 3^{2} * E^{2} \Lambda^{8} *\left(-275 / 2 / 1 N G^{3} * S I^{2}+7175 / 16 / 1 N G^{3} * S I^{4}-42675 / 64 / 1 N G^{3} * S I^{6}+14025 / 32 / 1 N G^{3} * 5 I^{8}-6875 / 64 / 1 m G^{3} * S I^{10}+105 / 4 / I H G^{2}-1825 / 16\right.$ $/ 1 N_{G}{ }^{2} * S I^{2}+19095 / 128 / 1 N_{G}{ }^{2} * S I^{4}-14635 / 256 / 1 N_{G}{ }^{2} * S I^{6}-1075 / 256 / I N_{G}{ }^{2} * 5 I^{8}-3 / I N G / 5 I^{2}+23 / 8 / I N G+12517 / 96 / I N_{G} * S I^{2} * 231293 / 760 / 1 N_{G} * 3 I^{4}$

- $+44285 / 256 /$ IHG*5I ${ }^{6}$,

+ A2*A4*E ${ }^{2} / L^{12} * 1675 / 2 / I N G^{4} * S I^{2}+87925 / 6 / 1 N G^{4} * S^{4} I^{4}-270525 / 4 / I N G^{4} * S I^{6}+1244625 / 8 / I N G^{4} * 5 I^{8}-360297975 / 2048 / 1 N G^{4} * 5 I^{10}+404468625 / 4096$ /ING ${ }^{4} * S I^{12}-90391875 / 4096 / I N G^{4} * S I^{14}-45 / 2 / 1 N G^{3}-3345 / 1 N G^{3} * S I^{2}+474895 / 16 / 1 N G^{3} * S I^{4}-11503925 / 128 / I N G^{3} * 5 I^{6}+258527725 / 2048 / 1 N G^{3} * 5 I^{6}$

$-86821625 / 1024 / \mathrm{ING}^{3} * \mathrm{SI}^{10}+22645875 / 1024 / \mathrm{ING}^{3} * 5 \mathrm{I}^{12}+1595 / \mathrm{B} / \mathrm{ING}^{2}-104725 / 32 / \mathrm{ING}^{2} * \mathrm{SI}^{2}+1616605 / 128 / \mathrm{ING}^{2} * 51^{4}-41058965 / 2048 / 1 \mathrm{NG}^{2} * \mathrm{SI}{ }^{6}$ $+58662445 / 4096 /$ ING $^{2} * S I^{8}-15718675 / 4096 /$ ING $^{2} * 5 I^{10}-1285 / 32 /$ IHG $+99905 / 128 / 1$ IHG *SI ${ }^{2}-6093709 / 2048 / I N G * S I^{4}+9538867 / 2048 / 1 N G * 5 I^{6}$ $-4370325 / 2048 / 11 H 6 * 5 I^{8}$,

$+A 2^{3} * E^{2} /^{12} *\left(15795 / 2 / I N G^{4} * S I^{2}-432675 / 8 / 1 H G^{4} * S I^{4}+154225 / I N G^{4} * S I^{6}-60002485 / 256 / 1 N G^{4} * S I^{8}+41 C 043675 / 2048 / 111 G^{4} * S I^{10}-46665375 / 512\right.$ $/$ ING $^{4} * 5 I^{12}+35375625 / 2048 / 1 N G^{4} * 5 I^{14}-1053 / 2 / I N G^{3}+72675 / 8 / I H G^{3} * 5 I^{2}-1342165 / 32 / 1 N G^{3} * 5 I^{4}+11390673 / 138 / 1 N G^{3} * 5 I^{6}-2015 B 9205 / 204 B$

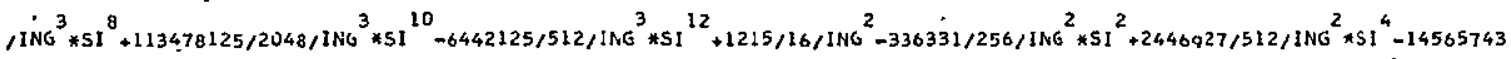
$\left.12048 / 1 \mathrm{NG}^{2} * 51^{6}+39294065 / 8192 / 1 \mathrm{NG}^{2} * S\right]^{8}-10086005 / 8192 / \mathrm{ING}^{2} * 51^{10}+3815 / 256 / 1 N G-1083 / 8 / 1 \mathrm{ING} * 5 I^{2}+97301 / 2048 / 1 N G * 51^{4}+42647 / 1 / 28 / I N G$ $* 5 I^{6}-1054795 / 4096 / I^{\prime H G H S I^{8}}$,

COEFFICIENT OF COSISG

$1 / A 2^{3} * A 3 * A 4^{2} * E / L^{10} *\left(-1000 / I N G^{3} * S I+37625 / 4 / 1 N G^{3} * S I^{3}-527375 / 16 / I N G^{3} * 5 I^{5}+1712375 / 32 / I N G^{3} * 5 I^{7}-1306375 / 32 / I N G^{3} * S I^{9}+189875 / 16 / 1 N G^{3}\right.$

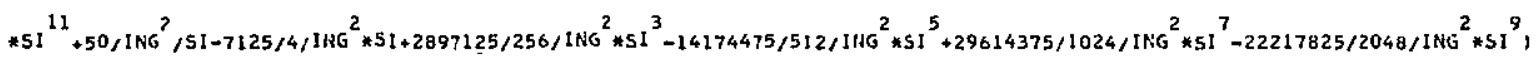
$+1 / A Z^{3} * A 3^{3} * \varepsilon / L^{6} *\left(25 / 2 / I N_{G}{ }^{2} * S I^{3}-25 / 1 N_{G}{ }^{2} * S I^{5}+25 / 2 / 1 H_{G}{ }^{2} * S 1^{7}-55 / 16 / 1 M_{G} * 51 * 505 / 32 / I N_{G} * S I^{3}-395 / 32 / I N G * S 1^{5}-1 / B / 51+53 / 16 * S 1-413 / 64\right.$ $* \mathrm{SI}^{3}$,

$+1 / A 2 * A 3 * A 4 * E / L^{10} *\left(3000 / 1 N G^{3} * S I-91475 / 4 / I N G^{3} * S I^{3}+519325 / 8 / I N G^{3} * S I^{5}-5623675 / 64 / 1 N G^{3} * S I^{7}+7356475 / 128 / 1 N G^{3} * S I^{9}-1875125 / 12 B / I N G^{3}\right.$

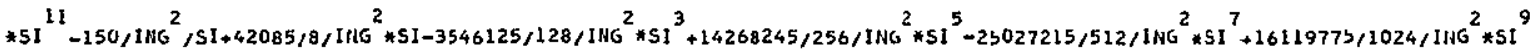

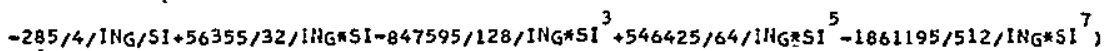
$+A 2 * A 3 * E / L^{10} *\left(-2160 / 1 N G^{3} * S I+25575 / 2 / 1 N G{ }^{3} * 5 I^{3}-478465 / 16 / 1 N G^{3} * S I^{5}+2214225 / 64 / I N G^{3} * 5 I^{7}-1269275 / 64 / 1 N G^{3} * 5 I I^{4}+144375 / 32 / 111 G^{3} * 5 I I^{11}\right.$

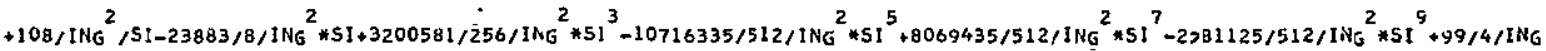

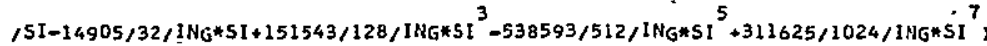

$\stackrel{*}{*} 3^{h}$ COEFFICIENT OF SIN(2*5G)

$1 / A 2^{3} * A 4^{3} * C I * E^{2} / L^{12} *\left(-5625 / 2 / I N G^{4} * 5 I^{2}+200625 / B / I N_{G}{ }^{4} * 5 I^{4}-2720625 / 32 / I N_{G}{ }^{4} * S I^{6}+4370625 / 32 / I M G{ }^{4} * 5 I^{8}-6706875 / 64 / I N G{ }^{4} * S I I^{10}\right.$ $47931875 / 256 / \mathrm{ING}^{4} * \mathrm{SI} \mathrm{I}^{12}+375 / 2 / \mathrm{ING}^{3}-13375 / 4 / \mathrm{ING}^{3} * 5 \mathrm{I}^{2}+544125 / 32 / \mathrm{ING}^{3} * 5 \mathrm{I}^{4}-291375 / 8 / \mathrm{ING}^{3} * 5 \mathrm{I}^{6}+2235625 / 64 / \mathrm{ING}^{3} * 5 \mathrm{I}^{\mathrm{a}}-1586375 / 128 / 1 \mathrm{NG}{ }^{3}$ $\stackrel{5: 10}{10}$ 
$+1 / A 2^{3} * A 3^{2} * A 4 * C I * E^{2} L^{8} * 1-125 / 2 / 1 N G G^{3} * S I^{2}+5625 / 16 / 1 N G^{3} * S I^{4}-17125 / 32 / I N G^{3} * S I^{6}+7875 / 32 / I M G^{3} * 51^{8}+75 / 4 / 1 N G{ }^{2}-925 / 16 / I N G^{2} * S 1^{2}-75 / 24$ $/ I N G^{2} * S I^{4}+22575 / 256 / I N G^{2} * S I^{6}-5 / 2 / I N G / 5 I^{2}+115 / 8 / 1 N_{G}+1425 / 32 / I N_{G} * S I^{2}-23275 / 256 / I N G * 5 I^{4}$,

$+1 / A 2 * A 4^{2} * C 1 * E^{2} L^{12} *\left(15375 / 2 / I N G{ }^{4} * 5 I^{2}-424975 / 8 / I N G^{4} * 5 I^{4}+4378125 / 32 / 1 N G^{4} * 51 I^{6}-42983625 / 256 / 1 M G^{4} * 5 I^{8} * 6349875 / 64 / 1 N G^{4} * 51{ }^{10}\right.$ $-23244375 / 1024 / \mathrm{ING}^{4} * S I^{12}-1025 / 2 / 1 \mathrm{NG}^{3}+57025 / 8 / \mathrm{ING}^{3} * \mathrm{SI}^{2}-212375 / 8 / \mathrm{ING}^{3} * \mathrm{SI}^{4}+2577075 / 64 / 1 \mathrm{NG}^{3} * S I^{6}-6771625 / 256 / 1 N \mathrm{NG}^{3} * 5 I^{6}+1561875$

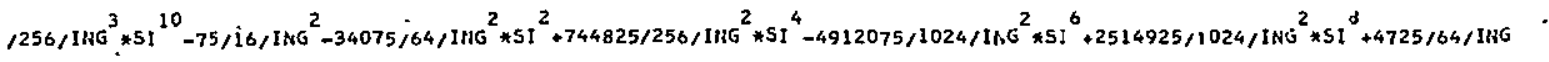
$-79725 / 128 / 1 N G * 5 I^{2}+1320165 / 1024 / I^{2} H * 5 I^{4}-24745 / 32 / I N G * S I^{6}$,

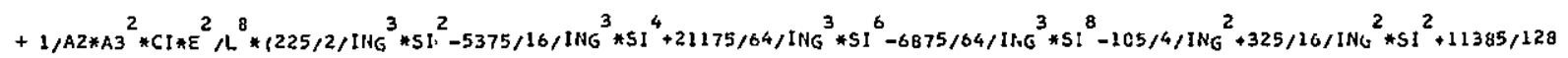
IING ${ }^{2} * S I^{4}-21975 / 256 / I N G^{2} * S I^{6}+3 / I N G / S I^{2}-12 / 8 / 1$ NG $-6115 / 96 / I N G * S I^{2}+18835 / 256 / I N G * S I^{4}$,

$+A 2 * A 4 * C I * E^{2} / L^{12} *\left(-675 / 2 / I N G^{4} * S I^{2}-90525 / 8 / 1 N G^{4} * S I^{4}+450525 / 8 / I N G{ }^{4} * S I^{6}-198525 / 2 / 1 N G^{4} * S I^{8}+157008375 / 2048 / 1 N G^{4} * S I I^{10}-90391875\right.$

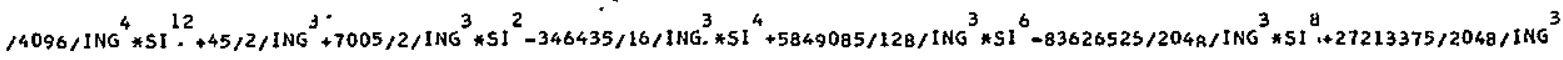
$* 511^{10}-1595 / 8 / I N G^{2}+50065 / 32 / I N O^{2} * S I^{2}-578465 / 128 / I N{ }^{2} * S I^{4}+8178645 / 2048 / I N G^{2} * 5 I^{6}-4756675 / 4096 / I N{ }^{2} * \pi 5 I^{6}+1285 / 32 / 1 N G-63925 / 128$ $\left./ I N G * 5 I^{2}+2602509 / 2048 / I N G * 5 I^{4}-875665 / 1024 / I N G * S I^{6}\right)$.

$+A 2^{3} * C I * \varepsilon^{2} \cdot L^{12} *\left(-15 / 95 / 2 / I^{4} G^{4} * 5 I^{2}+369495 / B / I N G^{4} * 5 I^{4}-864305 / 8 / I N G^{4} * S I^{6}+32344725 / 256 / I H G^{4} * 5 I I^{8}-151>85875 / 2048 / I N G^{4} * S I I^{10}\right.$. $+35375625 / 2048 / \mathrm{ING}^{4} * \mathrm{SI}^{12}+1053 / 2 / \mathrm{ING}^{3}-43191 / 8 / \mathrm{ING}^{3} * \mathrm{SI}^{2}+578209 / 32 / \mathrm{ING}^{3} * 51^{4}-3546285 / 128 / 1 \mathrm{MC}^{3} * \mathrm{SI}^{6}+41345525 / 2043 / \mathrm{ING}^{3} * \mathrm{SI}^{8}$

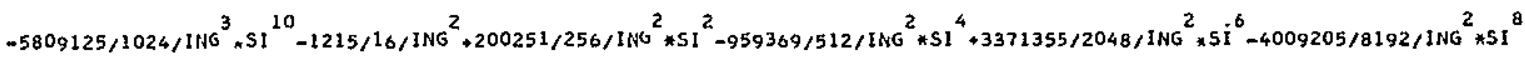
$-3815 / 256 / 1 N G+7951 / 256 / 1 N G * S I^{2}+157131 / 2048 / 1 N G * 51^{4}-210959 / 2048 / 1 N G * 5 I^{6}$, CUEFFICIENT OF $\cos (S G)$

$1 / \mathrm{AZ}^{3} * A 3 * A 4^{2} * C I * E / L^{10} *\left(1000 / I N G^{3} * S I-33625 / 4 / 11 G^{3} * S^{3} I^{3}+392875 / 16 / I N G^{3} * S I^{5}-926625 / 32 / I N G^{3} * 5 I^{7}+189 R 75 / 16 / 1 N G^{3} * S I^{9} * 50 / I N G^{2} / 5 I\right.$ $+5025 / 4 / \mathrm{ING}^{2} * 51-1558725 / 256 / \mathrm{ING}^{2} * S I^{3}+2570225 / 256 / \mathrm{ING}^{2} * S I^{5}-5415725 / 1024 / \mathrm{ING}^{2} * S I^{7}$,

$+1 / A 2^{3} * A 3^{3} * C I * E / L^{6} *\left(-25 / 2 / 1 M_{G}{ }^{2} * S I^{3}+25 / 2 / I M G{ }^{2} * S I^{5}+55 / 16 / I N G * 5 I-95 / 16 / 1 N G * 5 I I^{3}+1 / 8 / 51-9 / 4 * S I\right)$

$+1 / A 2 * A 3 * A 4 * C I * E / L^{10} * 1-3000 / 1 \mathrm{NG}^{3} * S I+79475 / 4 / 1 \mathrm{NG}^{3} * S I^{3}-360375 / 8 / \mathrm{ING}^{3} * S I^{5}+2740675 / 64 / 1 N G^{3} * S I^{7}-1875125 / 12 B / 1 N G^{3} * 51^{9}+150 / 1 \mathrm{NG}{ }^{2} / 5 I$

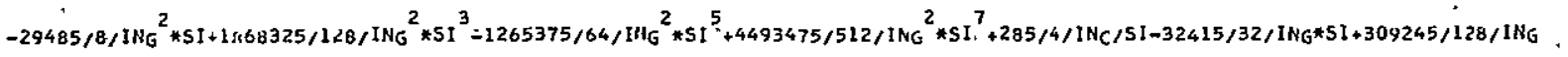
$* 51^{3}-394555 / 256 /$ ING*SI ${ }^{5}$,

*A2*A3*CI*F/ $L^{10} *\left\{2160 / 1 N G^{3} * S I-21255 / 2 / I N G^{3} * 5 I^{3}+308425 / 16 / I N G^{3} * S I^{5}-980525 / 64 / I N G^{3} * S I^{7}+144375 / 32 / T N G^{3} * 5 I I^{9}-108 / I N G G^{2} / 5 I+14811 / 8\right.$.

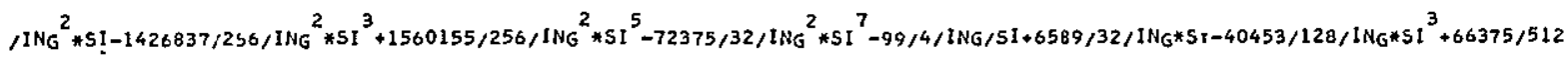
/ING*SI ${ }^{5}$,

B 7 Secular Perturbations

$\frac{\partial F^{* *}}{\partial(L, G, H)}=\frac{\partial F_{1}^{\star *}}{\partial(L, G, H)}+\frac{\partial F_{2}^{\star *}}{\partial(L, G, B)}+\frac{\partial F_{3}^{* *}}{\partial(L, G, H)}+\frac{\partial F_{4}^{\star \star}}{\partial(L, G, H)}$

B-52 
$\frac{\partial F_{1}^{\star *}}{\partial L}=\frac{3 J_{2}}{4 L^{4} G^{3}}\left(1-3 c^{2}\right)$

ORIGINAL PAGE IS

OF POOR QUALITH

$\frac{\partial F_{1}^{* *}}{\partial G}=\frac{3 J_{2}}{4 L^{3} G^{4}}\left(1-5 c^{2}\right)$

$\frac{\partial F_{1}^{* *}}{\partial H}=\frac{3 J_{2} c}{2 L^{3} G^{4}}$

$\frac{\partial F_{2}^{* *}}{\partial L}=\frac{3}{128 L^{4} G^{7}}\left\{u_{2}^{2}\left[15\left(1-2 c^{2}-7 c^{4}\right)-16 n\left(1-3 c^{2}\right)^{2}-5 n^{2}\left(5-18 c^{2}+5 c^{4}\right)\right]+15 u_{4} e^{2}\left(3-30 c^{2}+35 c^{4}\right)\right\}$

$\frac{\partial F_{2}^{\star *}}{2 G}=\frac{3}{328 L^{3} f^{8}}\left\{j_{2}^{2}\left[5\left(7-18 c^{2}-77 c^{4}\right)-24 n\left(1-3 c^{2}\right)\left(1-5 c^{2}\right)+n^{2}\left(-25+126 c^{2}-45 c^{4}\right)\right]+5 J_{4}\left[4\left(3-36 c^{2}+49 c^{4}\right)\right.\right.$

$\left.\left.+3 e^{2}\left[3-42 c^{2}+63 c^{4}\right)\right]\right\}$

$\frac{\partial F_{2}^{* \star}}{\partial H}=\frac{3 c}{32 L^{3} G^{3}}\left\{j_{2}^{2}\left[5\left(1+7 c^{2}\right)+12 n\left(-1+3 c^{2}\right)+n^{2}\left(-9+5 c^{2}\right)\right]+5 J_{4}\left(3-7 c^{2}\right)\left(2+3 e^{2}\right)\right\}$

$\frac{\partial F_{3}^{\star \star}}{\partial L}$

$1 / A 2 * A 4^{2} / L^{15} * 1675 / 128 / I N G * 5 I^{4}-1575 / 128 / I N G * S I^{6}+3675 / 512 / 1 H G * S I^{8}+3375 / 128 * E^{2} / I N G^{2} * S I^{4}-5625 / 64 * E^{2} / I N G^{2} * S I^{6}+49875 / 512 * E^{2} / 1 N G^{2}$ $* 51^{8}-18375 / 512 * E^{2}, I N G^{2} * S I^{10}-675 / 84 * E^{2}$ ING*SI ${ }^{2}+10125 / 128 * E^{2} / I N G * 5 I^{4}-8925 / 64 * E^{2} /\left[H G * 5 I^{6}+19375 / 25 * * E^{2}, I N G * S I^{8}\right.$,

$+1 / A 2 * A 3^{2} L^{11} *\left(-15 / 16 * 5 I^{2}+15 / 16 * 5 I^{4}-3 / 16 * I N G+9 / 32 * I N G * S I^{2}-15 / 16 * E^{2} * 5 I^{2}+15 / 16 * E^{2} * S I^{4}-3 / 16 * E^{2} * I N G * 27 / 64 * E^{2} * I H G * 5 I^{2}\right)$

$+A 2 * A 4 / L^{15} * 1505 / 64 / I N G * 5 I^{4}-2715 / 128 / I N G * S I^{6}+1575 / 12 B / 1 M G * 5 I^{8}-45 / 16+225 / 32 * S I^{2}+315 / 64 * 5 I^{4}-315 / 32 * 51^{6}+2925 / 64 * E^{2} / I H G^{2} * 5 I^{4} \cdot$ $-19425 / 12 B * E^{2} / I N G^{2} * S I^{6}+10725 / 64 * E^{2}, I N G^{2} * 5 I^{8}-7875 / 12 B * E^{2}, I N G^{2} * S 1 I^{10}-585 / 32 * E^{2}, I N G * S I^{2}+17775 / 128 * F{ }^{2} / I N G * S I^{4}-62775 / 256 * \varepsilon^{2}, I N G$ $* 51^{6}+64575 / 512 * E^{2} / I N G * 5 I^{8}+225 / 16 * E^{2}-7155 / 64 * E^{2} * 5 I^{2}+30915 / 128 * E^{2} * S 1^{4}-76545 / 512 * E^{2} * 51^{6}$,

$+A 2^{3} L^{15} *\left(507 / 12 B / 1\right.$ MG*SI ${ }^{4}-585 / 64 / 1 N G * S I^{6}+675 / 128 / 1 A G * S I^{8}-135 / 16+1023 / 32 * 51^{2}-2421 / 64 * 5 I^{4}+7485 / 512 * S I^{6}+2535 / 128 * E^{2} / 1 \mathrm{NG}^{2} * S I^{4}$ $-8385 / 128 * E^{2}, I M G^{2} * S I^{6}+9225 / 128 * E^{2}, I N G^{2} * S I^{8}-3375 / 12 B * E^{2}, I N G^{2} * S I^{10}-507 / 64 * E^{2}, I N G * S I^{2}+975 / 16 * E^{2}, I N G * S 1^{4}-27585 / 256 * E^{2} / I N G * S I^{6}$ $+14175 / 256 * E^{2} / I N G * S I^{8}-1665 / 32 * E^{2}+12393 / 64 * E^{2} * S I^{2}-119295 / 512 * E^{2} * 5 I^{4}+23265 / 256 * E^{2} * 5 I^{6}$,

$\frac{\partial F_{3}^{* *}}{\partial G}$

$1 / A 2 * A 4^{2} L^{15} * 1-675 / 128 / I N G * S I^{4}+1575 / 128 / I N G * S I^{6}-3675 / 512 / I N G * 5 I^{8}-3375 / 64 * E^{2} / I N_{G}{ }^{2} * 5 I^{4}+5625 / 32 * E^{2} / I N G^{2} * S I^{6}-49 B 75 / 256 * E^{2} / I N G^{2}$ $* S I^{8}+18375 / 256 * E^{2} / I N G^{2} * S I^{10}+675 / 32 * E^{2}, 1 N G * S I^{2}-42525 / 256 * E^{2} / 1 N G * S I^{4}+76125 / 256 * E^{2} / 1 N G * S I^{6}-15 B 025 / 1024 * E^{2} / I N G * S I^{8}$, 


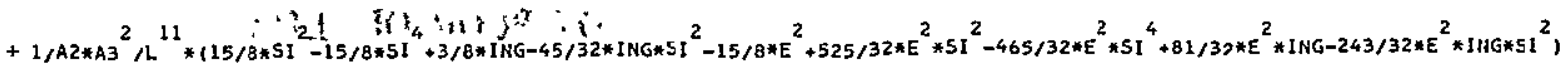
$+A 2 * A 4 / L^{15} *\left(-585 / 64 / I N G * S I^{4}+2715 / 128 / I N G * S I^{6}-1575 / 128 / I N G * S I^{8}+585 / 8-5625 / 16 * S I^{2}+64395 / 128 * S I^{4}-14595 / 64 * 5 I^{6}-2925 / 32 * E^{2} / I N G^{2}\right.$ $* 5 I^{4}+19425 / 64 * E^{2}, I 1 H G^{2} * S I^{6}-10725 / 32 * E^{2}, 1 N G^{2} * S I^{8}+7875 / 64 * E^{2}, I N G^{2} * S I^{10}+585 / 16 * E^{2} / I N G * 5 I^{2}-37035 / 128 * E^{2}, I N G * S I^{4}+66195 / 128 * E^{2}, I N G$ $* 51^{6}-137025 / 512 * E^{2}, I N G * S I^{8}+17325 / 32 * E^{2}-161415 / 64 * E^{2} * 5 I^{2}+894015 / 256 * E^{2} * S I^{4}-784035 / 512 * E^{2} * S I^{6}$, $+A 2^{3} / L^{15} *\left(-507 / 128 / I N G * S I^{4}+585 / 64 / I N G * S I^{6}-675 / 128 / I N G * S I^{8}-513 / 8+18 B 7 / 8 * 5 I^{2}-9531 / 32 * S I^{4}+63555 / 517 * 5 I^{6}-2535 / 64 * E^{2} / I N G^{2} * S I^{4}\right.$ $+8385 / 64 * E^{2} / I N G^{2} * 51^{6}-9225 / 64 * E^{2} / I N G^{2} * S I^{8}+3375 / 64 * E^{2} / 1126^{2} * S I^{10}+507 / 32 * E^{2} / I N G * S I^{2}-32253 / 256 * E^{2} / I N G * S I^{4}+57555 / 256 * E^{2} / I N G * S I^{6}$ $-7425 / 64 * E^{2}, I N G * S I^{d}-6351 / 16 * E^{2}+93939 / 54 * E^{2} * 5 I^{2}-481617 / 256 * E^{2} * 5 I^{4}+815355 / 1024 * E^{2} * 51^{6}$,

$\frac{\partial \mathrm{F}_{3}^{\star *}}{\partial \mathrm{H}}$ $1 / A 2 * A 4^{2} * C I / L^{15} *\left(3375 / 128 * E^{2} / I N G{ }^{2} * S I^{4}-7875 / 128 * E^{2} / 1 N G^{2} * S I^{6}+18375 / 512 * E^{2} / I N G{ }^{2} * S I^{8}-675 / 64 * E^{2} / I N G * S I^{2}+4725 / 12 \sigma^{*} E^{2} / I N G * S I^{4}-3675\right.$

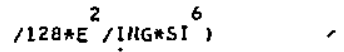

$+1 / A 2 * A 3^{2} * C I / L{ }^{11} *\left(-15 / 16 * 5 I^{2}-3 / 16 * 1 N G+15 / 8 * E^{2}-135 / 16 * E^{2} * 51^{2}-21 / 16 * E^{2} * 1 N G\right)$

$+A 2 * A 4 * C I / L^{15} *\left(-495 / 16+3105 / 32 * S I^{2}-9135 / 128 * S I^{4}+2925 / 64 * E^{2} / 1 H G^{2} * S I^{4}-13575 / 128 * E^{2} / I^{2}{ }^{2} * S I^{6}+7875 / 128 * E^{2} / I N G^{2} * S I^{8}-585 / 32 * E^{2} / I N G\right.$ $* S I^{2}+8145 / 125 * E^{2} / 1, G * S I^{4}-1575 / 32 * E^{2} / 1 N G * S I^{6}-3825 / 16 * E^{2}+45945 / 64 * E^{2} * S I^{2}-129465 / 256 * E^{2} * S I^{4}$, $+A 2^{3} * C I / L^{15} *\left(405 / 16-2091 / 32 * S I^{2}+333 / 8 * S I^{4}+2535 / 128 * \mathrm{~L}^{2} / I N G^{2} * S I^{4}-2925 / 64 * \mathrm{~L}^{2} / \mathrm{ING}^{2} * S I^{6}+3375 / 128 * \mathrm{E}^{2} / \mathrm{ING}{ }^{2} * S I^{8}-507 / 64 * \mathrm{E}^{2} / I H G * S I^{2}\right.$ $+1755 / 64 * E^{2}, I H G * S I^{*}-675 / 32 * E^{2} / I N G * S I^{6}+1263 / 8 * E^{2}-26523 / 64 * E^{2} * 5 I^{2}+137385 / 512 * E^{2} * 5 I^{4}$,

$\frac{\partial F_{4}^{* *}}{\partial L}$

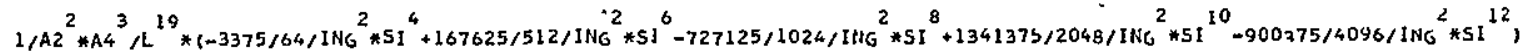
$+1 / A 2^{2} * A 3^{2} * A 4 / L^{15} * 1-225 / 64 / I N G * S I^{4}+975 / 12 B / I N G * S I^{6}-525 / 128 / 1 N G * 5 I^{8}+15 / 8-225 / 8 * S 1^{2}+2475 / 32 * 5 I^{4}-6825 / 128 * S I^{6}$,

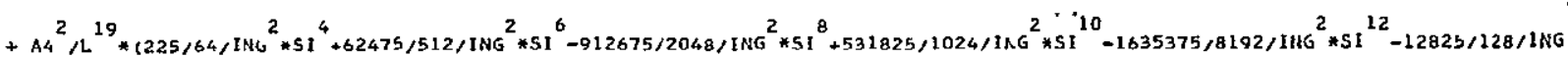

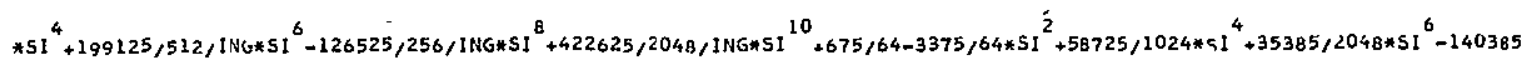
$14096 * \mathrm{SI}^{8}$,

$+A 3^{2} / L^{15} *\left(* 195 / 64 / I * G * S I^{4}+105 / 16 / I N G * 5 I^{6}-225 / 64 / I N G * S I^{8}+27 / 8-21 / 4 * 5 I^{2}-1113 / 128 * 5 I^{4}+345 / 32 * 5 I^{6}\right)$ $+A 2^{2} * A 4 / L^{19} * 17995 / 64 / I N G{ }^{2} * S I^{4}-267825 / 512 / 1 N G^{2} * S I^{6}+417315 / 512 / I N G^{2} * S I^{8}-1145325 / 2048 / 1 N G^{2} * S I^{10} * 291375 / 2048 / 1 N G^{2} * 5 I^{12}-9855 / 64$ IING*SI ${ }^{4}+295455 / 512 / I N G * S I^{6}-731235 / 1024 / I N G * 5 I^{8}+149625 / 512 / I N G * S I^{10}-1215 / 32+14775 / 64 * S I^{2}-19245 / 32 * 5 I^{4}+1401225 / 2048 * S^{6}$ $-1133265 / 4096 * 51^{8}$,

$+\mathrm{A}^{4} / \mathrm{L}^{19} *\left(4563 / 64 / 1 \mathrm{NG}^{2} * 5 I^{4}-169923 / 512 / \mathrm{NGG}^{2} * 5 I^{6}+1185585 / 2048 / 1 \mathrm{NG}^{2} * 5 I^{8} * 459225 / 1024 / \mathrm{NNG}^{2} * 5 I^{10}+266675 / 2048 / 1 \mathrm{NG}^{2} * \mathrm{SI}{ }^{12}-7449 / 128\right.$ IING*SI ${ }^{4}+6663 / 32 / I: G * S I^{6}-127215 / 512 / I_{\text {ING*SI }}^{8}+50625 / 512 / 1$ ING*SI ${ }^{10}-459 / 16+18213 / 128 * 5 I^{2}-234651 / 1024 * 5 I^{4}+543273 / 4096 * 5 I^{6}-68721$ $14096 * 51^{8}$, 


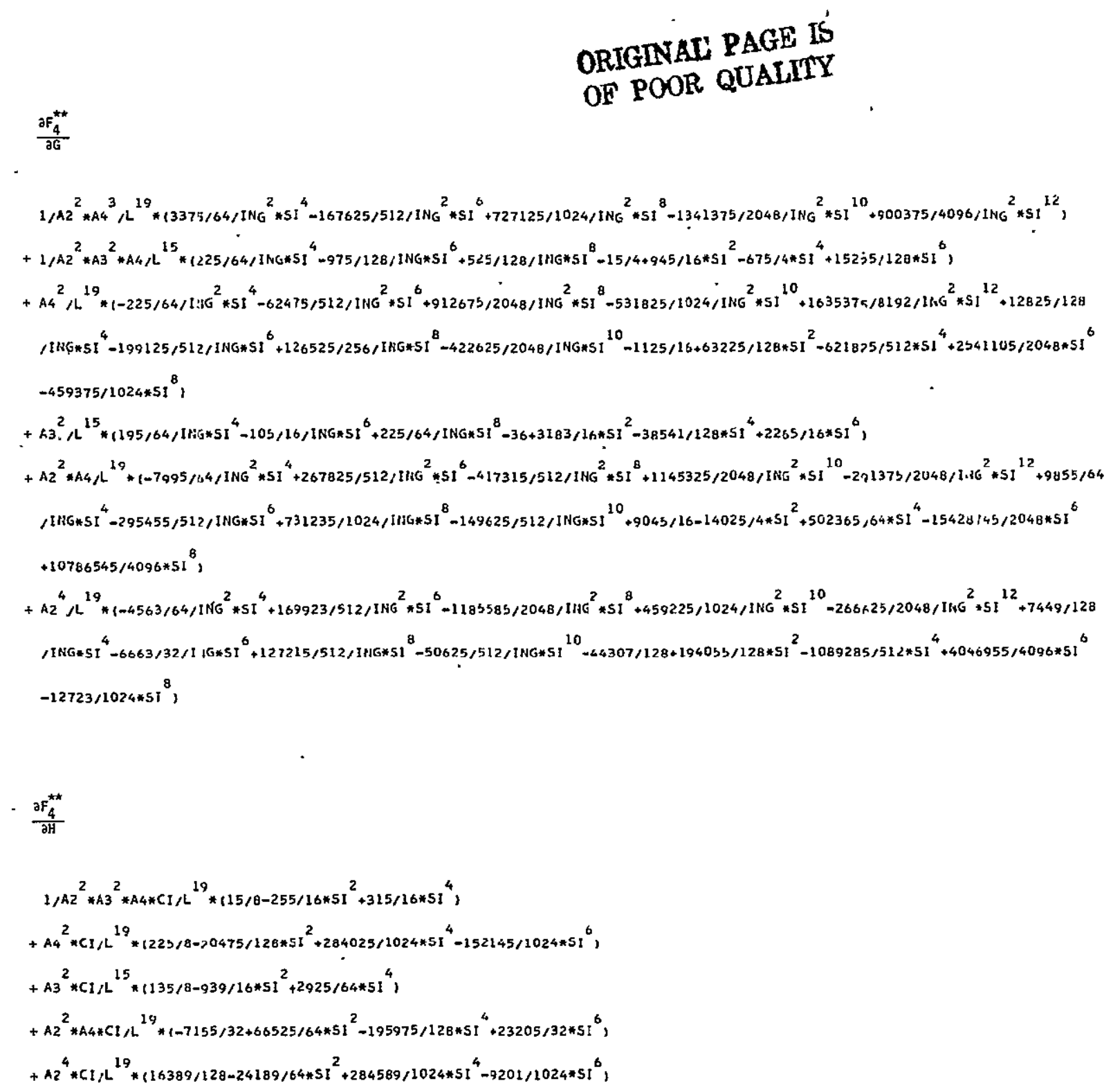




\section{NOTICE}

This series of Special Reports was instituted under the supervision of Dr. F. L. Whipple, Director of the Astrophysical Observatory of the Smithsonian Institution, shortly after the launching of the first artificial earth satellite on October 4, 1957. Contributions come from the Staff of the Observatory.

First is sued to ensure the immediate diss emination of data for satellite tracking, the reports have continued to provide a rapid distribution of catalogs of satellite observations, orbital information, and preliminary results of data analyses prior to formal publication in the appropriate journals. The Reports are also used extensively for the rapid publication of preliminary or special results in other fields of astrophysics.

The Reports are regularly distributed to all institutions participating in the U.S. space research program and to individual scientists who request them from the Publications Division, Distribution Section, Smithsonian Astrophysical Observatory, Cambridge, Massachusetts 02138 . 